\title{
VIRTUAL CYCLES OF GAUGED WITTEN EQUATION
}

\author{
GANG TIAN AND GUANGBO XU
}

\begin{abstract}
We construct virtual cycles on moduli spaces of perturbed gauged Witten equation over a fixed smooth $r$-spin curve, under the framework of [TX15]. Together with the wall-crossing formula proved in the companion paper [TX], it completes the construction of the correlation function for the gauged linear $\sigma$-model announced in [TX16] as well as the proof of its invariance.
\end{abstract}

\section{Contents}

1. Introduction 1

2. Gauged Witten Equations and Perturbations 4

3. Definition of the Correlation Function 10

4. Invariance of the Correlation Function 19

5. Proof of Proposition $4.8 \quad 22$

6. Proof of Proposition $4.7 \quad 28$

7. Topological Virtual Orbifolds and Virtual Cycles 34

References $\quad 58$

\section{INTRODUCTION}

This paper, together with a companion paper [TX], is the third input in a series of papers aiming at a mathematically rigorous theory of the gauged linear $\sigma$-model (GLSM) as Witten proposed in [Wit93], following the two papers [TX15, TX16]. GLSM provides a fundamental framework in many studies of mathematics and physics related to string theory; it is of crucial importance in Hori-Vafa's physical proof of mirror symmetry [HV00]. It is also closely related to Gromov-Witten theory. However, despite many efforts, a mathematical foundation of GLSM has not been completely established ${ }^{1}$ and is needed for many important applications, for example, a mathematical proof of the Landau-Ginzburg/Calabi-Yau correspondence, and a geometric understanding of mirror symmetry.

In [TX15, TX16], we initiated our project on constructing a mathematical theory of GLSM. The central objects are the so-called gauged Witten equation and its moduli space. The gauged Witten equation is a system combining both the Witten equation, which was used to construct the A-side theory of orbifold Landau-Ginzburg models

Date: May 14, 2019.

G. T. is supported by DMS-1607091 and a grant from NSFC. G. X. is supported by AMS-Simons Travel Grant.

${ }^{1}$ Here we mean the $\mathcal{N}=(2,2)$, A-model closed-string theory, with a nontrivial superpotential. 
[FJR08, FJR13, FJR], and the symplectic vortex equation, which was used to construct the Hamiltonian-Gromov-Witten invariants [Mun99, Mun03], [CGS00] and [MT09]. Roughly speaking, given a noncompact Kähler manifold $X$, an action on $X$ by a reductive Lie group $G^{\mathbb{C}}$ with a moment map $\mu: X \rightarrow \mathfrak{g}$, and a holomorphic function $W: X \rightarrow \mathbb{C}$ which is homogeneous with respect to the $G^{\mathbb{C}}$-action, the gauged Witten equation reads

$$
\left\{\begin{array}{r}
\bar{\partial}_{A} u+\nabla W(u)=0, \\
* F_{A}+\mu(u)=0,
\end{array}\right.
$$

where $A$ is a connection on a $G$-bundle $P$ over a Riemann surface $\Sigma$, and $u$ is a section $u: P \rightarrow X$.

The moduli space of gauge equivalence classes of solutions contains the information of GLSM that can be extracted mathematically. In [TX15] we have obtained crucial analytical results about the gauged Witten equation and its moduli space. These results will be recalled in due course in the main body of this paper.

As in Gromov-Witten theory, the correlation function of GLSM is the most important numerical output, which leads to many interesting algebraic structures. The (symplectic) Gromov-Witten invariants, which can naively be interpreted as "curve counting," were constructed on any symplectic manifolds (cf. [LT98b], [FO99], also see [RT95], [RT97] on semi-positive symplectic manifolds). One major difficulty of the construction is the lack of transversality, namely, the moduli spaces of holomorphic curves may not be smooth or of expected dimensions. One has to perturb the equation in certain way to obtain well-defined countings. This is a highly non-trivial problem, which becomes more sophisticated when involved with maps defined on singular domains and nontrivial automorphism groups.

The method of constructing a system of consistent perturbations for the stratified moduli space and extracting topological information (the virtual cycle) is often called the virtual technique. After [LT98b] and [FO99], different versions of the virtual technique have been developed (in the non-algebraic case). We refer the readers to [LT98c], [HWZ07, HWZ09a, HWZ09b], [CLW], [Joya, Joyb], [Par16], [MW15, MW17a, MW17b], [FOOO12, FOOO16, FOOOa, FOOOb] for more recent developments.

In [TX16] we outlined the definition of the correlation function assuming the existence and good properties of a virtual cycle. In this paper we give the details of the virtual cycle construction; in the companion paper [TX] we prove an important property of the virtual cycles. Here we briefly explain our results. Let $X$ be a Kähler manifold and $Q: X \rightarrow \mathbb{C}$ be a holomorphic function. Suppose there is a $\mathbb{C}^{*}$-action on $X$ making $Q$ homogeneous. Let $\tilde{X}=\mathbb{C} \times X$ have an induced action by $G=T^{2}$. Let $W: \tilde{X} \rightarrow \mathbb{C}$ be $W(p, x)=p Q(x)$. There is also a moment map $\mu: \tilde{X} \rightarrow$ Lie $G$. Then one can write down the gauged Witten equation (1.1) over a so-called $r$-spin curve, which is an ordinary punctured Riemann surface $\Sigma$ with an orbifold line bundle $L$ whose $r$-th tensor power is isomorphic to the canonical bundle twisted with the orbifold data. Moreover, we assume that there is a vector space $\mathbf{V}$ of holomorphic functions with nice properties in order to properly perturb the gauged Witten equation. Our first main theorem is the following (the same as Theorem 3.1). 
Theorem 1.1. Let $\mathcal{C}$ be a smooth $r$-spin curve with broad punctures $\mathrm{z}_{1}, \ldots, \mathrm{z}_{b}$. For an equivariant curve class $B$ (see Subsection 2.5), a collection of strongly regular perturbations $\underline{P}=\left(P_{1}, \ldots, P_{b}\right)$, and a choice of asymptotic constrains at broad punctures $\underline{\kappa}=\left(\kappa_{1}, \ldots, \kappa_{b}\right)$, the moduli space $\mathcal{M}_{P}(\mathcal{C}, B, \underline{\kappa})$ of perturbed gauged Witten equation over $\mathcal{C}$ admits an oriented virtual orbifold atlas. In particular, it has a virtual cardinality $\# \mathcal{M}_{\underline{P}}(\mathcal{C}, B, \underline{\kappa}) \in \mathbb{Q}$.

Here a perturbation is strongly regular if for each broad puncture $\mathrm{z}_{i} \in \Sigma$, a perturbed function $\tilde{W}_{i}$ is a holomorphic Morse function and all its critical values have distinct imaginary parts. These type of perturbations form a subset of the space $\mathbf{V}_{i}$ which is a complement of a real analytic hypersurface $\mathbf{V}_{i}^{\text {wall }} \subset \mathbf{V}_{i}$. Theorem 1.1 relies crucially on the results about compactness and Fredholm property proved in [TX15].

The correlation function is supposed to be a multilinear function on the state space associated to the GLSM space (see Section 3). As in [TX16], we define the correlation function by taking certain linear combination of the virtual cardinality $\# \mathcal{M}_{\underline{P}}(\mathcal{C}, B, \underline{\kappa})$. Since the virtual cardinality does depend on the choice of strongly regular perturbations, it is a nontrivial procedure to prove the following invariance property of the correlation function (the same as Theorem 4.1).

Theorem 1.2. The correlation function defined by (3.5) and linear extension is independent of the choice of strongly regular perturbations at broad punctures.

The main idea of proving Theorem 1.2 is to connect two strongly regular perturbations at a broad puncture $\mathrm{z}_{i}$ via a generic path in $\mathbf{V}_{i}$, where the path may cross the wall $\mathbf{V}_{i}^{\text {wall }}$ at isolated places, and to construct a virtual chain on the universal moduli space over this path. When crossing the wall, the so-called BPS soliton solutions form codimension one boundary of the universal moduli space. The contribution of the BPS soliton solutions gives a Picard-Lefschetz type wall-crossing formula (Theorem 4.6), which is a crucial step in deriving the invariance of the correlation function. The idea and the method are inspired by a similar argument in the Landau-Ginzburg Amodel theory (see [FJR]) and the work of the second named author and S. Schecter in finite-dimensional Morse theory [SX14]. The detailed proof of the wall-crossing formula, though, is deferred to [TX].

Both Theorem 1.1 and Theorem 1.2 rely on the construction of corresponding virtual cycle/chain in moduli spaces. Our construction uses a version of the virtual technique which originated in [LT98b]. The method from [LT98b] is topological, in the sense that the charts are only topological and the smoothness of coordinate changes is not needed. This differs from other methods, such as, the Kuranishi method or polyfold method, for which certain weak smoothness of coordinate changes has to be established. Usually, it is rather technical to establish the smoothness property required in those methods. On the other hand, in a recent work [Par16], J. Pardon introduced a virtual technique which does not require smoothness of coordinate changes. In contrast, we get the virtual cycles by constructing perturbed sections in a more classical and topological way, while Pardon has his virtual cycles constructed via homological algebra operations.

This paper is organized as follows. In Section 2 we recall the basic set-up in [TX15] of the gauged Witten equation and perturbations. In Section 3 we recall the linear Fredholm theory and then construct the virtual cycle in the case of strongly regular 
perturbations. This completes the definition of the correlation function. Sections $4-6$ discuss the proof of the invariance of the correlation function: in Section 4 we consider variations of strongly regular perturbations and state the wall-crossing formula. In Section 6 we discuss the orientation issue and (re)prove the Picard-Lefschetz formula in our context. The key wall-crossing formula is proved in the companion paper [TX].

During the preparation of this paper, there appeared [CLLL15] and [FJR18] which use algebraic methods to construct virtual cycles for GLSM in the absence of broad punctures. During the preparation and revision of this paper, we developed a different setting in which we can construct a cohomological field theory for much more general GLSM spaces in the so-called geometric phases (see [TX17, TX2]). Recently there also appeared $\left[\mathrm{CFG}^{+}\right]$which uses categorical construction of cohomological field theories for certain GLSM spaces.

Acknowledgements. The second named author would like to thank Mohammad Tehrani for many helpful discussions, Huai-Liang Chang for discussions about the algebraic geometry of GLSM, Mauricio Romo and Wei Gu for discussions about the physics of GLSM.

\section{Gauged Witten Equations and Perturbations}

In this section we recall the basic setup of gauged Witten equation given in [TX15]. We apologize for many changes of notations since we think the new notations are more convenient to use in this paper.

2.1. The target space. Let $X$ be a Kähler manifold and $Q: X \rightarrow \mathbb{C}$ be a holomorphic function, with a single critical point $\star \in X$. Suppose there is a holomorphic $\mathbb{C}^{\star}$-action on $X$ such that $Q$ is homogeneous of degree $r(r \geq 2)$, meaning that $Q(g x)=g^{r} Q(x)$ for $x \in X$ and $g \in \mathbb{C}^{*}$. We assume that the $\mathbb{C}^{*}$-action restricts to a Hamiltonian $S^{1}$ action, and let $\mu^{\prime}: X \rightarrow \mathbf{i} \mathbb{R}$ denote a moment map. The pair $(X, Q)$ is called an $L G$ (Landau-Ginzburg) space and the $\mathbb{C}^{*}$-action is referred to as the $R$-symmetry.

Denote the group $S^{1}$ for the R-symmetry by $G^{\prime}$. We call $(\tilde{X}, W, G)$ the $G L S M$ (gauged linear $\sigma$-model) space whose elements are given as follows.

(1) $\tilde{X}=\mathbb{C} \times X$. Its coordinates are denoted by $\tilde{x}=(p, x)$ and the factor $\mathbb{C}$ is equipped with the standard Kähler structure.

(2) $G=G^{\prime} \times G^{\prime \prime} \simeq G^{\prime} \times S^{1}$ acts on $\tilde{X}$ where the $G^{\prime}$-factor acts on the $x$ coordinate and the $G^{\prime \prime} \simeq S^{1}$-factor acts by $g^{\prime \prime}(p, x)=\left(\left(g^{\prime \prime}\right)^{-r} p, g^{\prime \prime} x\right)$. The $G$-action is Hamiltonian with a moment map

$$
\mu(p, x)=\left(\mu^{\prime}, \mu^{\prime \prime}\right):=\left(\mu^{\prime}(x), \mu^{\prime}(x)+\frac{\mathbf{i} r}{2}|p|^{2}-\tau\right) .
$$

Here $\tau \in \mathbf{i} \mathbb{R}$ is a constant, which we fix from now on.

(3) $W: \tilde{X} \rightarrow \mathbb{C}$ is defined as $W(p, x)=p Q(x)$, which is $\left(G^{\prime \prime}\right)^{\mathbb{C}}$-invariant where $\left(G^{\prime \prime}\right)^{\mathbb{C}}$ is the complexification of $G^{\prime \prime}$.

We make the same assumptions on the LG space as listed in [TX15, Hypothesis 2.2, $2.4]$ in order to guarantee the compactness of the moduli space. We do not need these assumptions explicitly in this paper, except for the following properness property.

Hypothesis 2.1. The functions $|\nabla Q|$ and $\mu^{\prime}$ are both proper on $X$. 
We identify the Lie algebras of $G^{\prime}$ and $G^{\prime \prime}$ with $\mathbf{i} \mathbb{R}$ and denote a vector of $\mathfrak{g}=\mathbf{L i e} G$ as $\xi=\left(\xi^{\prime}, \xi^{\prime \prime}\right) \in \mathfrak{g}$. Denote by $\mathcal{X}_{\xi} \in \Gamma(T X)$ the infinitesimal action of $\xi$. To define the vortex equation, one also needs to specify a metric on $\mathfrak{g}$. We do not simply take the product metric on $\mathfrak{g}=\mathfrak{g}^{\prime} \times \mathfrak{g}^{\prime \prime}$ but use the norm defined by

$$
\left|\left(\xi^{\prime}, \xi^{\prime \prime}\right)\right|^{2}=\lambda^{-1}\left|\xi^{\prime}\right|^{2}+\left|\xi^{\prime \prime}\right|^{2}
$$

for a sufficiently large $\lambda$. The reason is explained in [TX15].

2.2. Rigidified $r$-spin curves. We recall the notion of $r$-spin curves. Since we will only consider a fixed domain curve, it is more convenient to rephrase the definitions without using the language of orbifolds.

Definition 2.2. Fix $r \in \mathbb{Z}, r \geq 3$. An $r$-spin curve with $k$ markings is a tuple $\left(\Sigma, \mathbf{z}, \mathbf{m}, L^{\prime}, \phi^{\prime}\right)$ where $\Sigma$ is a compact Riemann surface, $\mathbf{z}=\left\{\mathrm{z}_{1}, \ldots, \mathrm{z}_{k}\right\}$ is a set of marked points, $\mathbf{m}$ is a collection of integers $m_{1}, \ldots, m_{k} \in\{0,1, \ldots, r-1\}, L^{\prime} \rightarrow \Sigma$ is a holomorphic line bundle, and $\phi^{\prime}$ is a line bundle isomorphism

$$
\phi^{\prime}:\left(L^{\prime}\right)^{\otimes r} \simeq K_{\Sigma} \otimes \mathcal{O}\left(\left(1-m_{1}\right) \mathrm{z}_{1}\right) \otimes \cdots \otimes \mathcal{O}\left(\left(1-m_{k}\right) \mathrm{z}_{k}\right) .
$$

Let $\mathcal{C}=\left(\Sigma, \mathbf{z}, \mathbf{m}, L^{\prime}, \phi^{\prime}\right)$ be an $r$-spin curve. Denote $\Sigma^{*}=\Sigma \backslash \mathbf{z}$. It follows from the definition that if $z$ is a local coordinate on $\Sigma^{*}$, then there exists a local holomorphic frame $e^{\prime}$ of $L^{\prime}$, unique up to $\mathbb{Z}_{r}$-action, such that $\phi^{\prime}\left(\left(e^{\prime}\right)^{\otimes r}\right)=d z$. Moreover, if $w$ is a local coordinate centered at $\mathrm{z}_{i} \in \mathbf{z}$, then there exists local frames $e_{i}^{\prime}$ of $L^{\prime}$ near $\mathrm{z}_{i}$, unique up to $\mathbb{Z}_{r}$-action, such that

$$
\phi^{\prime}\left(\left(e_{i}^{\prime}\right)^{\otimes r}\right)=w^{m_{i}} \frac{d w}{w} .
$$

We call $\gamma_{i}=\exp \left(2 m_{i} \pi \mathbf{i} / r\right)$ the monodromy of the $r$-spin structure at $\mathrm{z}_{i}$.

Now we introduce the important notions of broadness/narrowness. Notice that since $Q$ is homogeneous with respect to the $G^{\prime}$-action, for any $\gamma \in G^{\prime}$, the critical point $\star$ is contained in the fixed point set $X_{\gamma} \subset X$ of $\gamma: X \rightarrow X$.

Definition 2.3. Given $\gamma \in \mathbb{Z}_{r} \subset G^{\prime}$, let $X_{\gamma}$ be the fixed point set of $\gamma$ in the LG space. $\gamma$ is called broad (resp. narrow) if $X_{\gamma} \neq\{\star\}$ (resp. $X_{\gamma}=\{\star\}$ ). A marking $z_{i}$ of an $r$-spin curve is broad/narrow if the monodromy $\gamma_{i}$ is broad/narrow.

Definition 2.4. Given an $r$-spin curve $\mathcal{C}=\left(\Sigma, \mathbf{z}, \mathbf{m}, L^{\prime}, \phi^{\prime}\right)$. A rigidification of $\mathcal{C}$ at a broad marking $\mathrm{z}_{i}$ is a choice of local frame $e_{i}$ of $L$ near $\mathrm{z}_{i}$ satisfying (2.4). An $r$-spin curve with rigidifications at all its broad markings is called a rigidified $r$-spin curve.

The purpose of rigidifying the $r$-spin curve is the same as in [FJR], because to perturb the equation we have to break the symmetry $Q$ or $W$ has. From now on we fix an $r$-spin curve $\left(\Sigma, \mathbf{z}, \mathbf{m}, L^{\prime}, \phi^{\prime}\right)$ and a rigidification.

2.3. Perturbations. Now we state the assumption that allow us to perturb the gauged Witten equation.

Hypothesis 2.5. For each broad $\gamma \in \mathbb{Z}_{r}$, there exists a nonzero, finite dimensional complex vector space $\mathbf{V}_{\gamma}$ parametrizing certain $\gamma$-invariant holomorphic functions $F_{\gamma}$ : $X \rightarrow \mathbb{C}$ satisfying [TX15, Hypothesis 2.8], particularly the following conditions. 
(1) Each $F_{\gamma} \in \mathbf{V}_{\gamma}$ is the sum of homogeneous functions and each summand has uniformly bounded derivative on $X$.

(2) For each $a_{\gamma} \in \mathbb{C}^{*}$, there is a complex analytic subset $\mathbf{V}_{\gamma, a_{\gamma}}^{\text {sing }} \subset \mathbf{V}_{\gamma}$ such that for each $F_{\gamma} \in \mathbf{V}_{\gamma} \backslash \mathbf{V}_{\gamma, a_{\gamma}}^{\text {sing }}$, the restriction of the function

$$
\tilde{W}_{\gamma}(x, p)=W(x, p)-a_{\gamma} p+F_{\gamma}(x)
$$

to $\tilde{X}_{\gamma}$ is a holomorphic Morse function with finitely many critical points.

(3) For each $a_{\gamma} \in \mathbb{C}^{*}$ and $F_{\gamma} \in \mathbf{V}_{\gamma, a_{\gamma}}^{\text {sing }},\left|\nabla \tilde{W}_{\gamma}\right|$ is bounded away from zero outside a compact subset.

(4) The set $\mathbf{V}_{\gamma, a_{\gamma}}^{\text {wall }} \subset \mathbf{V}_{\gamma} \backslash \mathbf{V}_{\gamma, a_{\gamma}}^{\text {sing }}$ defined by the coincidence of the imaginary parts of two critical values of $\left.\tilde{W}_{\gamma}\right|_{\tilde{X}_{\gamma}}$ is a locally finite union of real analytic hypersurfaces.

For $j=1, \ldots, b$, denote $\mathbf{V}_{i}=\mathbf{V}_{\gamma_{i}}$ and $\tilde{\mathbf{V}}_{i}=\mathbb{C}^{*} \times \mathbf{V}_{i}$. Denote

$$
\tilde{\mathbf{V}}_{i}^{\text {sing }}=\left\{\left(a_{i}, F_{i}\right) \mid F_{i} \in \mathbf{V}_{\gamma_{i}, a_{i}}^{\text {sing }}\right\}, \tilde{\mathbf{V}}_{i}^{\text {wall }}=\left\{\left(a_{i}, F_{i}\right) \mid F_{i} \in \mathbf{V}_{\gamma_{i}, a_{i}}^{\text {wall }}\right\} .
$$

Definition 2.6. $P_{i}=\left(a_{i}, F_{i}\right) \in \tilde{\mathbf{V}}_{i}$ is called a perturbation of $W$ at the $j$-th broad puncture. It is called regular (resp. strongly regular) if $P_{i} \notin \tilde{\mathbf{V}}_{i}^{\text {sing }}$ (resp. $P_{i} \notin \tilde{\mathbf{V}}_{i}^{\text {wall }} \cup \tilde{\mathbf{V}}$ sing $)$. It is called small if it is close to the origin of $\tilde{\mathbf{V}}_{i}$. A perturbation to the gauged Witten equation over $\mathcal{C}$ is a collection

$$
\underline{P}=\left(P_{i}\right)_{j=1}^{b}=\left(a_{i}, F_{i}\right)_{j=1}^{b}
$$

where $P_{i}$ is a perturbation at the $j$-th broad puncture. It is called regular (resp. strongly regular) if each $P_{i}$ is regular (resp. strongly regular).

Let $P_{i}=\left(a_{i}, F_{i}\right)$ be a perturbation at $\mathrm{z}_{i}$. We write

$$
\tilde{W}_{i}=W-a_{i} p+F_{i}, \quad \quad \tilde{W}_{\tilde{X}_{i}}:=\left.\tilde{W}_{i}\right|_{\tilde{X}_{i}} .
$$

We introduce the following notations. For $t \in\left(G^{\prime}\right)^{\mathbb{C}}=\mathbb{C}^{*},{ }^{2}$ we denote

$$
\tilde{W}_{i}^{t}(x, p)=t^{r} \tilde{W}_{i}\left(t^{-1} x, p\right)=W-t^{r} a_{i} p+t^{r} F_{i}\left(t^{-1} x\right)
$$

Then we have

$$
(x, p) \in \operatorname{Crit}\left[\left.\tilde{W}_{i}\right|_{\tilde{X}_{i}}\right] \Longleftrightarrow(t x, p) \in \operatorname{Crit}\left[\left.\tilde{W}_{i}^{t}\right|_{\tilde{X}_{i}}\right] ; \quad \tilde{W}_{i}^{t}(t x, p)=t^{r} \tilde{W}_{i}(x, p) .
$$

Remark 2.7. The purpose of using perturbation is the same as in finite dimensional Morse theory of functions with degenerate singularities. There are alternative ways to develop the mathematical GLSM without perturbation. One possibility is to adapt the approach of Jake Solomon [Sol05], by treating the degenerate function $W$ directly. Another possibility is similar to the approach of Venugopalan [Ven15] of defining quasimap invairants, when we are in the geometric phase (see [TX17] and [TX2]).

\footnotetext{
${ }^{2}$ We were using the symbol $\delta$ instead of $t$ in the previous papers [TX15, TX16].
} 
2.4. Gauged Witten equation. The gauged Witten equation is the combination of the Witten equation and the symplectic vortex equation, where the latter requires an area form on the Riemann surface. Choose from now on a smooth area form on the closed surface $\Sigma$, which determines a Hodge star operator

$$
*: \Omega^{2}(\Sigma) \rightarrow \Omega^{0}(\Sigma) .
$$

On the other hand, we use a cylindrical metric to define Sobolev norms. For each $\mathrm{z}_{i}$, we have fixed a holomorphic coordinate $w$ centered at $\mathrm{z}_{i}$. We identify a cylindrical end $C_{i}$ with the cylinder $[0,+\infty) \times S^{1}$. The latter has coordinates $s+\mathbf{i} t=-\log w$. Choose an area form on $\Sigma^{*}$ whose restriction to $C_{i}$ coincides with $d s \wedge d t$. It induces a cylindrical metric on $\Sigma^{*}$. For $\tau>0, p>0$ and $k \geq 0$, for any open subset $U \subset \Sigma^{*}$, let $W_{\tau}^{k, p}(U)$ denote the weighted Sobolev space where the norm is defined as $\|f\|_{W_{\tau}^{k, p}(U)}:=\left\|h_{s}^{-\tau / 2} f\right\|_{W^{k, p}(U)}$. Here the latter Sobolev norm is taken with respect to the cylindrical metric on $U \subset \Sigma^{*}$.

From now on we fix $p>2$.

The last auxiliary data we need to specify is a Hermitian metric on $L^{\prime}$. Fix a reference Hermitian metrics $H^{\prime}$ on $\left.L^{\prime}\right|_{\Sigma^{*}}$, which is of class $W_{\text {loc }}^{1, p}$, such that for each puncture $\mathrm{z}_{i}$ with $m_{i}$ and $e_{i}^{\prime}$ given in (2.4), for some $\tau>0$,

$$
\log \left(|w|^{-m_{i} / r}\left\|e_{i}^{\prime}\right\|_{H^{\prime}}\right) \in W_{\tau}^{2, p}\left(C_{i}\right) .
$$

Let $P^{\prime} \rightarrow \Sigma^{*}$ be the unit circle bundle of $\left(L^{\prime}, H^{\prime}\right)$. On the other hand, we choose a smooth $G^{\prime \prime}$-bundle $P^{\prime \prime} \rightarrow \Sigma$. Define

$$
P:=P^{\prime} \underset{\Sigma^{*}}{\times} P^{\prime \prime} \rightarrow \Sigma^{*} .
$$

This is a principal bundle with structure group $G=G^{\prime} \times G^{\prime \prime}$. Since $G$ acts on $\tilde{X}$, we take the associated bundle over $\Sigma^{\star}$, denoted by $Y$.

2.4.1. The variables. The variables of the gauged Witten equation include connections on $P$, sections of $Y$, and a finite degrees of freedom called "frames" at broad markings. These variables transform via gauge transformations.

Let us first specify the group of gauge transformations. Notice that $G$ is abelian. For $\tau>0$, let $\mathcal{G}_{\tau}^{\prime}$ (resp. $\mathcal{G}_{\tau}^{\prime \prime}$ ) be the space of maps $g^{\prime}: \Sigma^{*} \rightarrow G^{\prime}$ (resp. $g^{\prime \prime}: \Sigma^{*} \rightarrow G^{\prime \prime}$ ) of class $W_{\text {loc }}^{2, p}$ such that the restrictions to each cylindrical end $C_{i}$ can be written as $g^{\prime}=\exp \left(\mathbf{i} h^{\prime}\right)$ (resp. $\left.g^{\prime \prime}=\exp \left(\mathbf{i} h^{\prime \prime}\right)\right)$ with $h^{\prime} \in W_{\tau}^{2, p}\left(\right.$ resp. $\left.h^{\prime \prime} \in W_{\tau}^{2, p}\right)$. Define $\mathcal{G}_{\tau}=\mathcal{G}_{\tau}^{\prime} \times \mathcal{G}_{\tau}^{\prime \prime}$. Then $\mathcal{G}_{\tau}$, $\mathcal{G}_{\tau}^{\prime}, \mathcal{G}_{\tau}^{\prime \prime}$ are Banach Lie groups. Let $\mathcal{G}$ (resp. $\mathcal{G}^{\prime}, \mathcal{G}^{\prime \prime}$ ) be the union of $\mathcal{G}_{\tau}$ (resp. $\left.\mathcal{G}_{\tau}^{\prime}, \mathcal{G}_{\tau}^{\prime \prime}\right)$ for all $\tau>0$ which is also a group.

Now we discuss the class of connections. First, let $A_{0}^{\prime}$ be the Chern connection on $P^{\prime}$ associated to the metric $H^{\prime}$. Let $\mathcal{A}_{\tau}^{\prime}$ be the space of connections on $P^{\prime}$ that can be obtained from $A_{0}^{\prime}$ by a complex gauge transformation of regularity $W_{\tau}^{2, p}$, and $\mathcal{A}^{\prime}$ be the union of $\mathcal{A}_{\tau}^{\prime}$ for all $\tau>0$. On the other hand, fix from now on trivializations of $\left.P^{\prime \prime}\right|_{C_{i}}$. Let $\mathcal{A}_{\tau}^{\prime \prime}$ be the space of connections on $P^{\prime \prime}$ of regularity $W_{\text {loc }}^{1, p}$ whose restrictions to each $C_{i}$ is of the form $d+\alpha^{\prime \prime}$ with $\alpha^{\prime \prime}$ a 1 -form of regularity $W_{\tau}^{1, p}$, and $\mathcal{A}^{\prime \prime}$ be the union of $\mathcal{A}_{\tau}^{\prime \prime}$ for all $\tau>0$. Denote $\mathcal{A}_{\tau}=\mathcal{A}_{\tau}^{\prime} \times \mathcal{A}_{\tau}^{\prime \prime}$ and $\mathcal{A}=\mathcal{A}^{\prime} \times \mathcal{A}^{\prime \prime}$. Then $\mathcal{G}_{\tau}$ acts on $\mathcal{A}_{\tau}$ and $\mathcal{G}$ acts on $\mathcal{A}$ via usual gauge transformations. 
Another variable of the gauge Witten equation is a section of $Y$, which is usually denoted by $u$. We allow arbitrarily weak regularity of $u$ at this moment. Later we will consider more specific asymptotic constrains on $u$.

The last set of variables are frames of $L^{\prime \prime}$ at markings. Let $\operatorname{Fr}_{i}:=\left\{\phi_{i}: G^{\prime \prime} \rightarrow P_{\mathrm{z}_{i}}^{\prime \prime}\right\}$ be the set of trivializations of $P_{\mathrm{z}_{i}}^{\prime \prime}$, which is an $S^{1}$-torsor. There is a left $G^{\prime \prime}$-action $e^{\mathbf{i} \theta} \cdot \phi_{i}=\phi_{i} \circ e^{\mathbf{i} \theta}$. Moreover, using the trivializations of $\left.P^{\prime \prime}\right|_{C_{i}}$ fixed from the last paragraph, we extend each $\phi_{i} \in \mathbf{F r}_{i}$ to a trivialization $\phi_{i}^{\bullet}: G^{\prime \prime} \times\left. C_{i} \rightarrow P^{\prime \prime}\right|_{C_{i}}$ such that $\left(e^{\mathbf{i} \theta} \phi_{i}\right)^{\bullet}=\phi_{i}^{\bullet} \circ e^{\mathbf{i} \theta}$. From now on we identify $\phi_{i} \in \mathbf{F r}_{i}$ with $\phi_{i}^{\bullet}$. Denote $\underline{\mathbf{F r}}=\prod_{j=1}^{b} \mathbf{F r}_{i}$ whose elements are denoted by $\underline{\phi}=\left(\phi_{1}, \ldots, \phi_{b}\right)$.

The variables of the gauged Witten equation are $\boldsymbol{u}=(A, u, \underline{\phi})$ as described above.

Notation 2.8. We will often need to consider the expression of a section $u \in \Gamma(Y)$ under local trivializations. Consider the trivialization of $\left.P^{\prime}\right|_{C_{i}}$ induced from the unitary frame $e_{i}^{\prime} /\left\|e_{i}^{\prime}\right\|_{H^{\prime}}$ where $e_{i}^{\prime}$ is the one in (2.4) (which is unique by the rigidification). Together with $\phi_{i} \in \mathbf{F r}_{i}$ it determines a trivialization of $\left.P\right|_{C_{i}}$ and hence $\left.Y\right|_{C_{i}}$, which, by abuse of notation, is still denoted by $\phi_{i}^{\bullet}$. Then for $u \in \Gamma(Y)$, we write $u_{i}^{\bullet}: C_{i} \rightarrow \tilde{X}$ the map induced from $u$ and $\phi_{i}^{\bullet}$.

2.4.2. The lift of the superpotential. We describe how to lift the superpotential $W$ to $Y$. Given $A=\left(A^{\prime}, A^{\prime \prime}\right) \in \mathcal{A}$. By the isomorphism $\phi^{\prime}$ in the $r$-spin structure (see 2.3), around each point $q \in \Sigma^{*}$, there exists local coordinate $z_{q}$ and local frame $e_{q}^{\prime}$ of $L^{\prime}$, which is holomorphic with respect to $A^{\prime}$, such that $\phi^{\prime}\left(\left(e_{q}^{\prime}\right)^{\otimes r}\right)=d z_{q}$. Let $e_{q}^{\prime \prime}$ be an arbitrary local frame of $P^{\prime \prime}$. Define

$$
\mathcal{W}_{A}\left(\left[e_{q}^{\prime}, e_{q}^{\prime \prime}, x\right]\right)=W(x) d z_{q}
$$

This lift only depends on $A^{\prime}$ but not $A^{\prime \prime}$.

The lift of perturbed superpotential does depend on both components of the connection and the frames. Upon choosing $\underline{P}$, denote the perturbation of $\mathcal{W}_{A}$ by $\tilde{\mathcal{W}}_{A, \phi}$. Instead of stating the full definition, for the purpose of the current paper, we only list a few properties of $\tilde{\mathcal{W}}_{A, \underline{\phi}}$. The details can be found in [TX15].

(1) $\tilde{\mathcal{W}}_{A, \underline{\phi}} \in \Gamma\left(Y, \pi^{*} K_{\Sigma^{*}}\right)$ depends on both $A \in \mathcal{A}$ and $\underline{\phi}$ smoothly.

(2) Given $g: \Sigma \rightarrow G$ with $g\left(\mathrm{z}_{i}\right)=\mathbf{I d}$, viewed as a gauge transformation on $P$, one has

$$
\tilde{\mathcal{W}}_{g^{*} A, \underline{\phi}}\left(g^{-1} x\right)=\tilde{\mathcal{W}}_{A, \underline{\phi}}(x)
$$

(3) Outside the cylindrical ends around broad markings, $\tilde{\mathcal{W}}_{A, \underline{\phi}}=\mathcal{W}_{A}$.

(4) There is a number $t_{A}$ depending smoothly on $A \in \mathcal{A}$, and, on a cylindrical end around a broad marking $\mathrm{z}_{i}$, there exist holomorphic frames $e_{i}^{\prime}, e_{i}^{\prime \prime}$ of $L^{\prime}, L^{\prime \prime}$, such that $\phi^{\prime}\left(\left(e_{i}^{\prime}\right)^{\otimes r}\right)=w^{m_{i}} d w / w$ and

$$
\tilde{\mathcal{W}}_{A, \underline{\phi}}\left(\left[e_{i}^{\prime}, e_{i}^{\prime \prime}, z, x\right]\right)=\tilde{W}_{i}^{t_{A}} \circ w^{\frac{m_{i}}{r}} .
$$

(Recall the notation $W_{i}^{t}$ from (2.5).) Moreover, if $q=\left(g_{1}^{\prime \prime}, \ldots, g_{b}^{\prime \prime}\right)$ where $g_{i}^{\prime \prime} \in G^{\prime \prime}$, then one has

$$
\left.\tilde{\mathcal{W}}_{A, \underline{q} \underline{\underline{ }} \underline{ }}\right|_{C_{i}}=\left.\tilde{\mathcal{W}}_{A, \underline{\underline{\phi}}}\right|_{C_{i}} \circ g_{i}^{\prime \prime}
$$


2.4.3. The gauged Witten equation. For the fibre bundle $Y \rightarrow \Sigma^{*}$, the Kähler structure on $\tilde{X}$ induces a Hermitian structure on the vertical tangent bundle $T^{\perp} Y$. On the other hand, for any continuous connection $A$, the tangent bundle $T Y$ splits as the direct sum of $T^{\perp} Y$ and the horizontal tangent bundle. The latter is isomorphic to $\pi^{*} T \Sigma^{*}$, therefore the connection induces an integrable almost complex structure on $Y$. The vertical differential of $\tilde{\mathcal{W}}_{A, \underline{\underline{\phi}}}$ is a section

$$
d \tilde{\mathcal{W}}_{A, \underline{\phi}} \in \Gamma\left(Y, \pi^{*} K_{\Sigma^{*}} \otimes\left(T^{\perp} Y\right)^{*}\right) .
$$

The Hermitian metric on $T^{\perp} Y$ induces a conjugate linear isomorphism $T^{\perp} Y \simeq\left(T^{\perp} Y\right)^{\star}$; the complex structure on $\Sigma^{*}$ also induces a conjugate linear isomorphism $K_{\Sigma^{*}} \simeq \Lambda_{\Sigma^{*}}^{0,1}$. Therefore we have a conjugate linear isomorphism

$$
\pi^{*} K_{\Sigma^{*}} \otimes\left(T^{\perp} Y\right)^{*} \simeq \pi^{*} \Lambda_{\Sigma^{*}}^{0,1} \otimes T^{\perp} Y .
$$

The image of $d \tilde{\mathcal{W}}_{A, \underline{\phi}}$ under this map is denoted by $\nabla \tilde{\mathcal{W}}_{A, \underline{\phi}} \in \Gamma\left(Y, \pi^{*} \Lambda_{\Sigma^{*}}^{0,1} \otimes T^{\perp} Y\right)$. The $\underline{P}$-perturbed gauged Witten equation reads

$$
\left\{\begin{array}{c}
\bar{\partial}_{A} u+\nabla \tilde{\mathcal{W}}_{A, \underline{\varphi}}(u)=0 \\
* F_{A}+\mu^{*}(u)=0 .
\end{array}\right.
$$

Each term in the system is defined as follows: the connection $A$ induces a continuous splitting $T Y \simeq T^{\perp} Y \oplus \pi^{*} T \Sigma^{*}$ and $d_{A} u \in W_{\text {loc }}^{1, p}\left(T^{\star} \Sigma^{\star} \otimes u^{\star} T^{\perp} Y\right)$ is the covariant derivative of $u$; the $G$-invariant complex structure $J$ induces a complex structure on $T^{\perp} Y$ and $\bar{\partial}_{A} u$ is the $(0,1)$-part of $d_{A} u$ with respect to this complex structure. $\nabla \tilde{\mathcal{W}}_{A, \Phi}(u)$ is the pull-back of $\nabla \tilde{\mathcal{W}}_{A, \phi}$ by $u$, which lies in the same vector space as $\bar{\partial}_{A} u$. $F_{A} \in \Omega^{2}\left(\Sigma^{*}\right) \otimes \mathfrak{g}$ is the curvature form of $A, *: \Omega^{2}\left(\Sigma^{*}\right) \rightarrow \Omega^{0}\left(\Sigma^{*}\right)$ is the Hodge-star operator with respect to the smooth metric on $\Sigma$; the moment map $\mu$ lifts to a $\mathfrak{g}$-valued function on $Y$ and $\mu^{*}(u)$ is the dual of $\mu(u)$ with respect to the metric defined by $(2.2)$.

2.5. Asymptotic behavior and compactness. In [TX15] we proved that if $\boldsymbol{u}=$ $(A, u, \underline{\phi})$ is a finite energy solution to $(2.7)$ with bounded image (which we called a bounded solution), then $u$ has good asymptotic behavior at punctures. More precisely, let $(s, t)$ be the cylindrical coordinates on $C_{i}$. If $\mathrm{z}_{i}$ is narrow, i.e., the fixed point set of the monodromy acting on $X$ is isolated, then there is $\tilde{p}_{i}=\left(\star, p_{i}\right) \in \tilde{X}$ s.t.

$$
\lim _{z \rightarrow \mathrm{z}_{i}} e^{\frac{\mathrm{i} m_{i} t}{r}} u_{i}^{\bullet}(s, t)=\tilde{p}_{i} ;
$$

if $\mathrm{z}_{i}$ is broad, then there is a critical point $\kappa_{i}=\left(x_{i}, p_{i}\right) \in \operatorname{Crit}\left[\left.\tilde{W}_{i}\right|_{\tilde{X}_{i}}\right]$ such that

$$
\lim _{z \rightarrow \mathrm{z}_{i}} e^{\frac{\mathrm{i} m_{i} t}{r}} u_{i}^{\bullet}(s, t)=\kappa_{i}^{t_{A}}:=\left(t_{A} x_{i}, p_{i}\right) .
$$

Moreover, the rate of convergence is exponential, i.e., can be controlled by $e^{-\tau_{0} s}$ for some $\tau_{0}>0$. A consequence of this result is that any bounded solution defines a homology class $B \in H_{2}^{G}(\tilde{X} ; \mathbb{Q})$. We call a class in $H_{2}^{G}(\tilde{X} ; \mathbb{Q})$ an equivariant curve class ${ }^{3}$.

The behavior of the perturbed gauged Witten equation near broad punctures calls for the study of solitons, i.e., solutions over the infinite cylinder. Let $\gamma_{i}=\exp \left(\frac{\mathbf{i} 2 \pi m_{i}}{r}\right)$ be

\footnotetext{
${ }^{3}$ When $X=\mathbb{C}^{N}$, an equivariant curve class is essentially the degrees of $L^{\prime}$ and $L^{\prime \prime}$.
} 
the monodromy at $\mathrm{z}_{i}$ and $\tilde{W}_{i}$ be the perturbed superpotential. For $t \in(0,1)$, consider the following equation for maps $\sigma_{i}: \mathbb{R} \times S^{1} \rightarrow \tilde{X}$.

$$
\frac{\partial \sigma_{i}}{\partial s}+J\left[\frac{\partial \sigma_{i}}{\partial t}+\mathcal{X}_{\mathbf{i} m_{i} / r}\left(\sigma_{i}\right)\right]+\nabla \tilde{W}_{i}^{t}\left(e^{\frac{\mathbf{i} m_{i} t}{r}} \sigma_{i}\right)=0
$$

Since $\tilde{W}_{i}$ is $\gamma_{i}$-invariant, $\nabla \tilde{W}_{i}^{t}\left(e^{m_{i} \frac{\mathrm{i} t}{r}} \sigma_{i}\right)$ is well-defined over $\mathbb{R} \times S^{1}$. A bounded solution to $(2.8)$ is called a soliton, for which we know there are $\kappa_{i, \pm} \in \operatorname{Crit}\left[\tilde{W}_{\tilde{X}_{i}}^{t}\right]$ such that

$$
\lim _{s \rightarrow \pm \infty} e^{\frac{\mathbf{i} m_{i} t}{r}} \sigma_{i}(s, t)=\kappa_{i, \pm}
$$

Then we have the energy identity

$$
\left\|\frac{\partial \sigma_{i}}{\partial s}+J\left[\frac{\partial \sigma_{i}}{\partial t}+\mathcal{X}_{\mathbf{i} m_{i} / r}\left(\sigma_{i}\right)\right]\right\|_{L^{2}\left(\mathbb{R} \times S^{1}\right)}^{2}=2 \pi\left[\tilde{W}_{i}^{t}\left(\kappa_{i,-}\right)-\tilde{W}_{i}^{t}\left(\kappa_{i,+}\right)\right] .
$$

We have known from [TX15] that nontrivial solitons exist only when $\tilde{W}_{i}$ is not strongly regular, i.e., $\left.\tilde{W}_{i}\right|_{\tilde{X}_{i}}$ has two different critical values with identical imaginary part.

We call a soliton $\sigma_{i}$ a BPS soliton if $e^{\mathrm{i} m_{i} t / r} \sigma_{i}(s, t)$ is independent of $t$ for all $s \in \mathbb{R}$; otherwise it is called a non-BPS soliton. If $\sigma_{i}$ is a BPS soliton, then it is easy to see that $e^{\mathbf{i} m_{i} t / r} \sigma_{i}(s, t) \in \tilde{X}_{i}$ and is a negative gradient flow line of the real part of $\left.\tilde{W}_{i}^{t}\right|_{\tilde{X}_{i}}$.

Fixing an equivariant curve class $B$, a regular perturbation $\underline{P}$, and a collection of critical points $\underline{\kappa}=\left(\kappa_{1}, \ldots, \kappa_{b}\right)$. Let $\hat{\mathcal{M}}_{P}^{*}(\mathcal{C}, B, \underline{\kappa})$ be the the set of solutions to the $\underline{P}-$ perturbed gauged Witten equation over $\mathcal{C}$ that represent $B$ and have asymptotic limits at broad markings labelled by $\underline{\kappa}$. By the gauge symmetry of the perturbed gauged Witten equation, one can define the moduli space

$$
\mathcal{M}_{\underline{P}}^{*}(\mathcal{C}, B, \underline{\kappa})=\hat{\mathcal{M}}_{\underline{P}}^{*}(\mathcal{C}, B, \underline{\kappa}) / \mathcal{G} .
$$

By the compactness result of [TX15], $\mathcal{M}_{\underline{P}}^{*}(\mathcal{C}, B, \underline{\kappa})$ can be compactified by adding soliton solutions. Let $\mathcal{M}_{\underline{P}}(\mathcal{C}, B, \underline{\kappa})$ be the compactified moduli space. Moreover, the notion of sequential convergence defined in [TX15] induces a topology on $\mathcal{M}_{\underline{P}}(\mathcal{C}, B, \underline{\kappa})$, in the same way as Gromov-Witten theory (see [MS04, Section 5.6]) which is Hausdorff and compact. In particular, when $\underline{P}$ is strongly regular, no soliton solutions need to be added and $\mathcal{M}_{\underline{P}}^{*}(\mathcal{C}, B, \underline{\kappa})=\mathcal{M}_{\underline{P}}(\mathcal{C}, B, \underline{\kappa})$.

\section{Definition of the Correlation Function}

The definition of the GLSM correlation function given in [TX16, Section 3] relies on the following theorem. For the precise meanings of virtual orbifold atlas and the virtual cardinality, see the section7.

Theorem 3.1. Given a smooth $r$-spin curve $\mathcal{C}$, an equivariant curve class $B$, a strongly regular perturbation $\underline{P}=\left(P_{1}, \ldots, P_{b}\right)$, and a choice of asymptotic constrains at broad punctures $\underline{\kappa}$, the moduli space $\mathcal{M}_{\underline{P}}(\mathcal{C}, B, \underline{\kappa})$ admits an oriented virtual orbifold atlas. In particular, when the virtual dimension is zero, there is a well-defined virtual cardinality

$$
\# \mathcal{M}_{\underline{P}}(\mathcal{C}, B, \underline{\kappa}) \in \mathbb{Q} .
$$

Moreover, when $\mathcal{C}$ has at least one broad marking, one has

$$
\# \mathcal{M}_{\underline{P}}(\mathcal{C}, B, \underline{\kappa}) \in \mathbb{Z} .
$$


Theorem 3.1 will be proved momentarily, in Subsections 3.2-3.4. Before doing that we first recall the definition of the correlation function.

\subsection{Definition of the correlation function.}

\subsubsection{The state space.}

Definition 3.2. Let $\gamma$ be an element of $\mathbb{Z}_{r}$.

(1) If $\gamma$ is narrow, then the $\gamma$-sector of the state space $\mathcal{H}_{\gamma}$ is a 1 -dimensional $\mathbb{Q}$-vector space, generated by an element $e_{\gamma}$.

(2) If $\gamma$ is broad, then the $\gamma$-sector of the state space is

$$
\mathcal{H}_{\gamma}:=H^{\operatorname{mid}}\left(X_{Q_{\gamma}}^{c} / \mathbb{C}^{*} ; \mathbb{Q}\right) \text {. }
$$

Here $Q_{\gamma}$ is the restriction of $Q$ to $X_{\gamma}$ and $X_{Q_{\gamma}}^{c}:=X_{\gamma} \backslash Q_{\gamma}^{-1}(0)$.

(3) The total GLSM state space is

$$
\mathcal{H}_{\mathrm{GLSM}}:=\mathcal{H}_{Q}:=\bigoplus_{\gamma \in \mathbb{Z}_{r}} \mathcal{H}_{\gamma}
$$

Suppose $\gamma$ is broad. Since $Q_{\gamma}:=\left.Q\right|_{X_{\gamma}}: X \rightarrow \mathbb{C}$ is homogeneous of degree $r$, for any $a \in \mathbb{C}^{\star}$, the inclusion $Q_{\gamma}^{a}:=Q_{\gamma}^{-1}(a) \hookrightarrow X_{Q_{\gamma}}^{c}$ induces a diffeomorphism

$$
Q_{\gamma}^{a} / \mathbb{Z}_{r} \simeq X_{Q_{\gamma}}^{c} / \mathbb{C}^{*}
$$

as orbifolds. By the basic property of cohomology of orbifolds and equivariant cohomology, we know that

$$
\mathcal{H}_{\gamma} \simeq H^{\operatorname{mid}}\left(Q_{\gamma}^{a} ; \mathbb{Q}\right)^{\mathbb{Z}_{r}} .
$$

The monodromy action on the cohomology $H^{*}\left(Q_{\gamma}^{a} ; \mathbb{Q}\right)$ induced from the locally trivial fibration $Q_{\gamma}: X_{Q_{\gamma}}^{c} \rightarrow \mathbb{C}^{*}$ is equivalent to operator on $H^{*}\left(Q_{\gamma}^{a} ; \mathbb{Z}\right)$ induced from the $\mathbb{Z}_{r^{-}}$ action on $Q_{\gamma}^{a}$. Therefore, $\mathcal{H}_{\gamma}$ is the monodromy invariant part of the middle dimensional rational cohomology of a regular fibre of $Q_{\gamma}$.

For each $\gamma \in \mathbb{Z}_{r}$, we assume that there exists a perfect pairing $\langle\cdot, \cdot\rangle_{\gamma}: \mathcal{H}_{\gamma} \otimes \mathcal{H}_{\gamma} \rightarrow \mathbb{Q}$ such that with respect to the obvious isomorphism $\varepsilon: \mathcal{H}_{\gamma} \rightarrow \mathcal{H}_{\gamma^{-1}},\langle\cdot, \cdot\rangle_{\gamma}=\langle\varepsilon \cdot, \varepsilon \cdot\rangle_{\gamma^{-1}}$. In many situations one has a naturally defined perfect pairing. Indeed, we have the following $\mathbb{Z}_{r}$-equivariant exact sequence

$$
H^{*-1}\left(X_{\gamma}\right) \longrightarrow H^{*-1}\left(Q_{\gamma}^{a}\right) \longrightarrow H^{*}\left(X_{\gamma}, Q_{\gamma}^{a}\right) \longrightarrow H^{*}\left(X_{\gamma}\right)
$$

If the arrow in the middle is an isomorphism in $\mathbb{Q}$-coefficients for $*=n_{\gamma}$ (it is the case when $\left.X=\mathbb{C}^{N}\right)$, then one can define a pairing between $H^{\text {mid }}\left(Q_{\gamma}^{a} ; \mathbb{Q}\right)$ and $H^{\text {mid }}\left(Q_{\gamma}^{-a} ; \mathbb{Q}\right)$ as in [FJR13, Page 36]. Nonetheless, the pairing defines an isomorphism

$$
\mathcal{O} \mapsto \mathcal{O}^{*}: \mathcal{H}_{\gamma} \mapsto \mathcal{H}_{\gamma}^{*} \simeq H_{\text {mid }}\left(Q_{\gamma}^{a} ; \mathbb{Q}\right)^{\mathbb{Z}_{r}}
$$

Remark 3.3. One can shift the degrees of states in $\mathcal{H}_{\gamma}$ by the convention of Chen-Ruan cohomology. However in this paper we do not do that explicitly; indeed, in this paper, we are skipping all discussions about the dimensions of the virtual cycles as well as the degrees of states. They can be easily recovered from the index formula proved in [TX15]. 
3.1.2. Vanishing cycles in $Q_{\gamma}^{a}$. The use of Lagrange multiplier requires us to consider the complex Morse theory on $Q_{\gamma}^{a}$. If we have a holomorphic Morse function $F: Q_{\gamma}^{a} \rightarrow \mathbb{C}$, then the unstable or stable manifolds of its critical points (with respect to the negative gradient flow of the real part of $\left.F\right|_{Q_{\gamma}^{a}}$ ) represent certain $\infty$-relative cycles. For any compact subset $Z \subset X$, consider the relative homology $H_{*}\left(Q_{\gamma}^{a}, Q_{\gamma}^{a} \backslash Z\right)$. The inverse limit with respect to the direct system of compact subsets under inclusion is denoted by $H_{*}\left(Q_{\gamma}^{a}, \infty\right)$. This is the dual space of $H_{c}^{*}\left(Q_{\gamma}^{a}\right)$. Then we have the intersection pairing

$$
\cap: H_{\text {mid }}\left(Q_{\gamma}^{a}\right) \otimes H_{\text {mid }}\left(Q_{\gamma}^{a}, \infty\right) \rightarrow \mathbb{Z}
$$

Now take a strongly regular perturbation $P_{i}=\left(a_{i}, F_{i}\right) \in \tilde{\mathbf{V}}_{i}$. Abbreviate $Q_{\gamma_{i}}$ by $Q_{i}$ and $Q_{i}^{-1}\left(a_{i}\right)$ by $Q_{i}^{a_{i}}$. Consider the negative gradient flow of $\boldsymbol{R e} F_{i}$ restricted to $Q_{i}^{a_{i}}$. For each critical point $\kappa_{i}$ of $\left.F_{i}\right|_{Q_{i}^{a_{i}}}$, denote by

$$
\left[W_{\kappa_{i}}^{u}\right] \in H_{\text {mid }}\left(Q_{i}^{a_{i}}, F_{i}^{-\infty} ; \mathbb{Z}\right)\left(\operatorname{resp} .\left[W_{\kappa_{i}}^{s}\right] \in H_{\text {mid }}\left(Q_{i}^{a_{i}}, F_{i}^{+\infty} ; \mathbb{Z}\right)\right)
$$

the class of the unstable (resp. stable) manifold of this flow. Here

$$
F_{i}^{+\infty}=Q_{i}^{a_{i}} \cap\left(\boldsymbol{\operatorname { R e }} F_{i}\right)^{-1}([M,+\infty)), F_{i}^{-\infty}=Q_{i}^{a_{i}} \cap\left(\boldsymbol{\operatorname { R e }} F_{i}\right)^{-1}((-\infty,-M])
$$

for some $M>0$. We still use $\left[W_{\kappa_{i}}^{u / s}\right]$ to denote their images under the map

$$
H_{\text {mid }}\left(Q_{i}^{a_{i}}, F_{i}^{ \pm \infty} ; \mathbb{Z}\right) \rightarrow H_{\text {mid }}\left(Q_{i}^{a_{i}}, \infty ; \mathbb{Z}\right)
$$

3.1.3. The correlation function. The correlation function is a multilinear map

$$
\langle\cdot\rangle_{B}: \bigotimes_{j=1}^{k} \mathcal{H}_{\gamma_{i}} \rightarrow \mathbb{Q}
$$

To define (3.4), we choose the last $n$ inputs (narrow states) to be the generators of the corresponding sectors $\mathcal{O}_{i}=e_{\gamma_{i}} \in \mathcal{H}_{\gamma_{i}}, j=b+1, \ldots, b+n$. Suppose the first $b$ inputs (the broad states) are $\mathcal{O}_{i} \in \mathcal{H}_{\gamma_{i}}, j=1, \ldots, b$. Then define

$$
\left\langle\mathcal{O}_{1}, \ldots, \mathcal{O}_{b}, \mathcal{O}_{b+1}, \ldots, \mathcal{O}_{b+n}\right\rangle_{B, \underline{P}}:=\sum_{\underline{\kappa}} \# \mathcal{M}_{\underline{P}}(\mathcal{C}, B, \underline{\kappa}) \cdot \prod_{j=1}^{b} \mathcal{O}_{i}^{*} \cap\left[W_{\kappa_{i}}^{u}\right] .
$$

Here $\# \mathcal{M}_{\underline{P}}(\mathcal{C}, B, \underline{\kappa}) \in \mathbb{Q}$ is the virtual cardinality of Theorem 3.1 , and $\mathcal{O}_{i}^{*} \in H_{\text {mid }}\left(Q_{i}^{a_{i}} ; \mathbb{Q}\right)^{\mathbb{Z}_{r}}$ is the image of $\mathcal{O}_{i}$ under (3.2) and the $\cap$ is the intersection (3.3). By linear extension, this provides a correlation function which a priori depends on the choice of the strongly regular perturbation $\underline{P}$. Eventually we will prove the independence of $\underline{P}$, thus (3.4) is well-defined.

3.2. Review the linear theory. Now we start to rigorously define the virtual cardinality $\# \mathcal{M}_{\underline{P}}(\mathcal{C}, B, \underline{\kappa})$, or, more generally, a virtual cycle on $\mathcal{M}_{\underline{P}}(\mathcal{C}, B, \underline{\kappa})$. For the first step we review the linear Fredholm theory for the perturbed gauged Witten equation over $\mathcal{C}$.

As before, $\mathcal{C}=\left(\Sigma, \mathbf{z}, \mathbf{m}, L^{\prime}, \phi^{\prime}\right)$ is a smooth $r$-spin curve with first $b$ markings broad and last $n$ markings narrow. Let $\tau>0, k \geq 0$. Let $E \rightarrow \Sigma^{*}$ be a vector bundle. Let $W_{\tau}^{k, p}\left(\Sigma^{*}, E\right)$ be the space of $W_{\text {loc }}^{k, p}$ sections such that its restriction on each cylindrical end is of class $W_{\tau}^{k, p}$. Here the Sobolev norms is taken with respect to some fixed choice 
of connection on $E$. We drop $\Sigma^{*}$ from the notation in this section and abbreviate the space by $W_{\tau}^{k, p}(E)$. When $k=0$ we also denote it by $L_{\tau}^{p}(E)$.

Definition 3.4. Given an equivariant curve class $B, \underline{\kappa}$, and a small $\tau>0$, we define

$$
\mathcal{B}:=\mathcal{B}_{\tau}^{1, p}(B, \underline{\kappa}) \subset \mathcal{A}_{\tau}^{1, p} \times W_{\text {loc }}^{1, p}\left(\Sigma^{*}, Y\right) \times \underline{\mathbf{F r}},
$$

which consists of tuples $\boldsymbol{u}=(A, u, \underline{\phi})$ satisfying the following conditions.

(1) For each broad $\mathrm{z}_{i}, u$ has the asymptotic limit $\kappa_{i}$ at $\mathrm{z}_{i}$ (see Subsection 2.5).

(2) For each narrow $\mathrm{z}_{i}, u_{i}^{\bullet}$ is asymptotic to some $\tilde{p}_{i}=\left(\star, p_{i}\right) \in \tilde{X}_{i}$.

(3) The above two conditions implies that $u$ extends to an orbifold section over $\mathcal{C}$ and we require that the section represents the equivariant curve class $B$.

The rate of convergence of $u_{i}^{\bullet}$ to its limit is in $W_{\tau}^{1, p}$.

$\mathcal{B}$ is a Banach manifold. A general point is denoted as $\boldsymbol{u}=(A, u, \underline{\phi})$. Define a Banach vector bundle $\mathcal{E} \rightarrow \mathcal{B}$ whose fibre over $\boldsymbol{u}$ is

$$
\mathcal{E}_{u}^{-}:=L_{\tau}^{p}\left(\Sigma^{*}, \Lambda^{0,1} \otimes u^{*} T^{\perp} Y\right) \oplus L_{\tau}^{p}\left(\Sigma^{*}, \mathfrak{g}\right) .
$$

3.2.1. Deformation theory and gauge fixing. Let $\mathcal{G}_{\tau}$ be the set of $W_{\text {loc }}^{2, p}$ maps from $\Sigma^{*}$ to $G$ that are asymptotic to the identity at all punctures, and such that the converges at a marking is in $W_{\tau}^{2, p}$. Then $\mathcal{G}_{\tau}$ acts on $\mathcal{B}$ on the first two components $A$ and $u$ by gauge transformations. Given $\boldsymbol{u} \in \mathcal{B}$, the linearizations of the gauge transformation and the gauged Witten equation induce the deformation complex

$$
\operatorname{Lie} \mathcal{G} \longrightarrow T_{\boldsymbol{u}} \mathcal{B} \longrightarrow \mathcal{E}_{\boldsymbol{u}}^{-}
$$

A usual way of considering problems with gauge symmetry is to take special slices of the action by the group of gauge transformations. In our case the gauge group is abelian, so one can transform all connections to a common slice. More precisely, choose from now on a smooth reference connection $A_{0} \in \mathcal{A}_{\tau}^{1, p}$. Let $-k$ be the index of the operator $\Delta: W_{\tau}^{2, p}(\mathfrak{g}) \rightarrow L_{\tau}^{p}(\mathfrak{g})$. Choose $s_{1}, \ldots, s_{k} \in C_{0}^{\infty}\left(\Sigma^{*}, \mathfrak{g}\right) \subset L_{\tau}^{p}(\Sigma, \mathfrak{g})$ such that

$$
\operatorname{Image}\left(\Delta: W_{\tau}^{2, p}(\mathfrak{g}) \rightarrow L_{\tau}^{p}(\mathfrak{g})\right)+\operatorname{Span}\left\{s_{1}, \ldots, s_{k}\right\}=L_{\tau}^{p}(\mathfrak{g})
$$

Denote $\Lambda_{G F}=\operatorname{Span}\left\{s_{1}, \ldots, s_{k}\right\}$ and denote the following composition by $\overline{d^{*}}$.

$$
T \mathcal{A}_{\tau}^{1, p} \stackrel{d^{*}}{\longrightarrow} L_{\tau}^{p}(\mathfrak{g}) \longrightarrow \frac{L_{\tau}^{p}(\mathfrak{g})}{\Lambda_{G F}}
$$

$A$ is said to be in Coulomb gauge (relative to $\left.A_{0}\right)$ if $\overline{d^{\star}}\left(A-A_{0}\right)=0$. From the definition it is easy to see that any connection in $\mathcal{A}_{\tau}^{1, p}$ can be transformed via a gauge transformation in $\mathcal{G}_{\tau}$ to a unique connection in Coulomb gauge relative to $A_{0}$.

Incorporating the gauge fixing condition, we define a section of a bundle over $\mathcal{B}$ which describes the deformation theory of the gauged Witten equation. Define a Banach space bundle $\mathcal{E} \rightarrow \mathcal{B}$ whose fibre over $\boldsymbol{u}=(A, u, \underline{\phi}) \in \mathcal{B}$ is

$$
\mathcal{E}_{\boldsymbol{u}}=L_{\tau}^{p}\left(\Lambda^{0,1} \otimes u^{*} T^{\perp} Y\right) \oplus L_{\tau}^{p}(\mathfrak{g}) \oplus\left(L_{\tau}^{p}(\mathfrak{g}) / \Lambda_{G F}\right) .
$$

Denote the last summand by $\overline{L_{\tau}^{p}(\mathfrak{g})}$. Define $\mathcal{F}: \mathcal{B} \rightarrow \mathcal{E}$ by $\mathcal{F}(\boldsymbol{u})=(\mathcal{W}(\boldsymbol{u}), \mathcal{V}(\boldsymbol{u}))$ where

$$
\mathcal{W}(\boldsymbol{u})=\bar{\partial}_{A} u+\nabla \tilde{\mathcal{W}}_{A, \underline{\phi}}(u) \in L_{\tau}^{p}\left(\Lambda^{0,1} \otimes u^{\star} T^{\perp} Y\right)
$$




$$
\mathcal{V}(\boldsymbol{u})=\left(* F_{A}+\mu(u), \overline{d^{\star}}\left(A-A_{0}\right)\right) \in L_{\tau}^{p}(\mathfrak{g}) \oplus \overline{L_{\tau}^{p}(\mathfrak{g})} .
$$

The linearized operator at $\boldsymbol{u}$ is a bounded linear operator $D_{\boldsymbol{u}} \mathcal{F}: T_{\boldsymbol{u}} \mathcal{B} \rightarrow \mathcal{E}_{\boldsymbol{u}}$.

\section{Proposition 3.5.}

(1) $\mathcal{B}$ is a Banach manifold, whose tangent space at $\boldsymbol{u}=(A, u, \underline{\phi})$ is isomorphic to

$$
T_{\boldsymbol{u}} \mathcal{B} \simeq T \mathcal{A}_{\tau}^{1, p} \oplus W_{\tau}^{1, p}\left(u^{*} T^{\perp} Y\right) \oplus \mathbb{C}^{n} \oplus \mathbb{R}^{b} .
$$

(2) $\mathcal{G}_{\tau}$ acts smoothly on $\mathcal{B}$ such that the isomorphism (3.6) is equivariant in a natural way. When there is at least one puncture on $\mathcal{C}$, the $\mathcal{G}_{\tau}$-action is free.

(3) For each curve class $B$, there exists $\tau=\tau_{B}>0$ satisfying the following condition. For any bounded solution $(A, u, \underline{)})$ to the perturbed gauged Witten equation over $\mathcal{C}$ with curve class $B$, there exist a gauge transformation $g \in \mathcal{G}$ and $\underline{\kappa}=\left(\kappa_{1}, \ldots, \kappa_{b}\right)$ such that $g^{*}(A, u, \underline{\phi}) \in \mathcal{B}_{\tau}^{1, p}(B, \underline{\kappa})$.

(4) $\overline{\mathcal{E}} \rightarrow \mathcal{B}$ is a Banach space bundle and $\mathcal{F}: \mathcal{B} \rightarrow \mathcal{E}$ is a smooth section. For any $\boldsymbol{u} \in \mathcal{B}$, the linearized operator $D_{\boldsymbol{u}} \mathcal{F}: T_{\boldsymbol{u}} \mathcal{B} \rightarrow \mathcal{E}_{\boldsymbol{u}}$ is Fredholm.

(5) The natural map $\mathcal{F}^{-1}(0) \rightarrow \mathcal{M}_{\underline{P}}^{*}(\mathcal{C}, B, \underline{\kappa})$ is a homeomorphism.

Remark 3.6. This proposition has been almost proved in [TX15] so here we only explain what was missing there. For (i), the difference from the case of [TX15] is that we shall vary the framing at broad punctures. This gives the additional $\mathbb{R}^{b}$-factor in (3.6). For (ii), we know that an automorphism of a connection is a constant gauge transformation. When there are punctures, $\mathcal{G}$ contains no constants other than the identity.

3.3. The orientation of $D_{\boldsymbol{u}} \mathcal{F}$. (See Section 6 for related discussions.) By an orientation of a Fredholm operator $F: E \rightarrow E^{\prime}$ we mean an orientation of the determinant line $\operatorname{det} F:=\operatorname{det} \operatorname{Ker} F \otimes(\operatorname{det} \operatorname{Coker} F)^{\vee}$. An orientation of a continuous family of Fredholm operators $F_{x}: E_{x} \rightarrow E_{x}^{\prime}$ for $x \in N$ is a continuous trivialization of the determinant line bundle $\operatorname{det} F \rightarrow N$. Hence if there is a homotopy of Fredholm operators $F_{x, t}: E_{x} \rightarrow E_{x}^{\prime}$, $t \in[0,1]$, then the orientability problem of the family $F_{x, 0}$ is equivalent to that of $F_{x, 1}$.

In our case, consider the family of linearized operators $D_{\boldsymbol{u}} \mathcal{F}: T_{\boldsymbol{u}} \mathcal{B} \rightarrow \mathcal{E}_{\boldsymbol{u}}$ for $\boldsymbol{u} \in \mathcal{B}$. The first component of the gauged Witten equation also gives another family of operators $D_{\boldsymbol{u}} \mathcal{W}: W_{\tau}^{1, p}\left(u^{\star} T^{\perp} Y\right) \rightarrow L_{\tau}^{p}\left(\Lambda^{0,1} \otimes u^{\star} T^{\perp} Y\right)$.

Lemma 3.7. The family $\left\{D_{\boldsymbol{u}} \mathcal{F}\right\}_{\boldsymbol{u} \in \mathcal{B}}$ is oriented if and only the family $\left\{D_{\boldsymbol{u}} \mathcal{W}\right\}_{\boldsymbol{u} \in \mathcal{B}}$ is oriented, and there is a canonical identification between their orientations.

Proof. The finite rank part of $D_{\boldsymbol{u}} \mathcal{F}$ coming from the last two summands of (3.6) doesn't affect the orientability problem. So one only needs to consider following operator at $a=1$.

$$
\left[\begin{array}{l}
\beta \\
v
\end{array}\right] \mapsto\left[\begin{array}{c}
D_{\boldsymbol{u}} \mathcal{W}(v)+a D_{\boldsymbol{u}}^{\prime} \mathcal{W}(\beta) \\
* d \beta+a \nu d \mu(u) \cdot v \\
\overline{d^{\star}} \beta
\end{array}\right] .
$$

Here $\beta$ and $v$ are infinitesimal deformations of $A$ and $u$, respectively, $a \in[0,1]$ and $D_{\boldsymbol{u}}^{\prime} \mathcal{W}$ is the partial derivative of $\mathcal{W}$ in the $\beta$ direction. By varying a from 1 to 0 , this family of operators remain Fredholm and hence the orientation is reduced to the case of $a=0$. Moreover, the operator $\beta \mapsto\left(* d \beta, \overline{d^{*}} \beta\right)$ is independent of $\boldsymbol{u}$, hence is naturally orientable. Therefore, the orientability is reduced to the family $\left\{D_{\boldsymbol{u}} \mathcal{W}\right\}_{\boldsymbol{u} \in \mathcal{B}}$. 
Next, notice that $\mathcal{B}$ is not a product, but a fibre bundle: the condition on the section $u$ depends on the connection $A$ and the framing $\underline{\phi}$. We denote by $\mathcal{B}_{A, \underline{\phi}}(\underline{\kappa}) \subset \mathcal{B}(\underline{\kappa})$ be the subset consisting of $(A, u, \underline{\phi})$ with fixed $(A, \underline{\phi})$. Moreover, since $A$ lies in a contractible space, which doesn't affect the orientability. Hence we omit the dependence of $A$ and denote by $\mathcal{B}(\underline{\phi}, \underline{\kappa})$ be the space of all such $(A, u, \underline{\phi})$ 's. Then the orientability of $\left\{D_{\boldsymbol{u}} \mathcal{W}\right\}_{\boldsymbol{u} \in \mathcal{B}(\underline{\underline{\phi}}, \underline{\kappa})}$ is similar to the case of ordinary Gromov-Witten and Floer theory. The orientation can be chosen in a coherent way in the sense of [FH93]. More precisely, it means the following. For each critical point $\kappa_{i} \in \operatorname{Crit}\left[\left.\tilde{W}_{i}\right|_{\tilde{X}_{\gamma_{i}}}\right]$, one can choose an orientation on the unstable manifold $W_{\kappa_{i}}^{u}$. Then for two critical points $\kappa_{i}, \kappa_{i}^{\prime}$, choose a curve $l_{i}: \mathbb{R} \rightarrow \tilde{X}_{i}$ such that $\left.l_{i}\right|_{(-\infty,-1]}=\kappa_{i}$ and $\left.l_{i}\right|_{[1,+\infty)}=\kappa_{i}^{\prime}$. Define

$$
\tilde{l}_{i}: \mathbb{R} \times S^{1} \rightarrow \tilde{X}, \tilde{l}_{i}(s, t)=e^{-m_{i} \frac{\mathrm{i} t}{r}} l_{i}(s) .
$$

Then by gluing $u \in \mathcal{B}(\underline{\phi}, \underline{\kappa})$ with $\tilde{l}_{i}$ in a consistent way, one obtains a continuous map

$$
\underline{l}: \mathcal{B}(\underline{\phi}, \underline{\kappa}) \rightarrow \mathcal{B}\left(\underline{\phi}, \underline{\kappa}^{\prime}\right) \text {. }
$$

Let the determinant line bundle be $\mathcal{L} \rightarrow \mathcal{B}(\underline{\varphi}, \underline{\kappa})$ and $\mathcal{L}^{\prime} \rightarrow \mathcal{B}\left(\underline{\varphi}, \underline{\kappa^{\prime}}\right)$ respectively.

On the other hand, the choice of orientations on the unstable manifolds of $\kappa_{i}$ and $\kappa_{i}^{\prime}$ induces an orientation of the operator

$$
D_{l_{i}}: W^{1, p}\left(\mathbb{R}, l_{i}^{*} T \tilde{X}_{i}\right) \rightarrow L^{p}\left(\mathbb{R}, l_{i}^{*} T \tilde{X}_{i}\right), D_{l_{i}}(\xi)=\nabla_{s} \xi+\nabla^{2} \tilde{W}_{i}\left(l_{i}\right) \cdot \xi
$$

and hence an orientation of the linearization of the soliton equation along $\tilde{l}_{i}$ (for details see Section 6 ). The gluing process induces an isomorphism $\underline{l}_{*}: \mathcal{L} \rightarrow \underline{l}^{*} \mathcal{L}^{\prime}$ of the determinant line bundles (well-defined up to homotopy).

Definition 3.8. An orientation of the family $\left\{D_{\boldsymbol{u}} \mathcal{W}\right\}_{\boldsymbol{u} \in \mathcal{B}(\underline{\underline{\phi}}, \underline{\kappa})}$ and an orientation of the family $\left\{D_{\boldsymbol{u}^{\prime}} \mathcal{W}\right\}_{\boldsymbol{u}^{\prime} \in \mathcal{B}\left(\underline{\phi}, \underline{\kappa}^{\prime}\right)}$ are coherent with respect to the orientations on the unstable manifolds of all $\kappa_{i}$ and $\kappa_{i}^{\prime}$, if they are consistent with the isomorphism $\underline{l}_{*}$.

The following proposition follows from the well-known results of Floer-Hofer [FH93].

Proposition 3.9. Fix $\underline{\phi}$ and $\underline{\kappa}$.

(1) The family $\left\{D_{\boldsymbol{u}} \mathcal{W}\right\}_{\boldsymbol{u} \in \mathcal{B}(\underline{\phi}, \underline{\kappa})}$ is orientable.

(2) If we choose orientations on the unstable manifolds $W_{\kappa_{i}}^{u}$ for all $i$, then there is a canonically induced orientation on $\left\{D_{\boldsymbol{u}} \mathcal{W}\right\}_{\boldsymbol{u} \in \mathcal{B}(\underline{\phi}, \underline{\kappa})}$. If we change the orientation on one $W_{\kappa_{i}}^{u}$, then the orientation of $\left\{D_{\boldsymbol{u}} \mathcal{W}\right\}_{\boldsymbol{u} \in \mathcal{B}(\underline{\underline{\alpha}}, \underline{\kappa})}$ changes.

(3) Let $\underline{\kappa}^{\prime}=\left(\kappa_{1}^{\prime}, \ldots, \kappa_{b}^{\prime}\right)$ be another set of critical points and choose orientations on $W_{\kappa_{i}}^{u}$ and $W_{\kappa_{i}^{\prime}}^{u}$ for all $i$. Then the induced orientation on $\left\{D_{\boldsymbol{u}} \mathcal{W}\right\}_{\boldsymbol{u} \in \mathcal{B}(\underline{\phi}, \underline{\kappa})}$ and the induced orientation on $\left\{D_{\boldsymbol{u}^{\prime}} \mathcal{W}\right\}_{\boldsymbol{u}^{\prime} \in \mathcal{B}\left(\underline{\phi}, \underline{\kappa}^{\prime}\right)}$ are coherent.

Lastly, we need to show that the orientation bundle over the space of all $\underline{\phi}$ is trivial. It suffices to consider the variation of one $\phi_{i}$. It is easy to see that changing $\phi_{i}$ to $e^{\mathbf{i} \theta} \phi_{i}$ is the same as fixing $\phi_{i}$ while changing $\left(a_{i}, F_{i}\right)$ to $\left(e^{\mathbf{i} r \theta} a_{i}, F_{i} \circ e^{-\mathbf{i} \theta}\right)$. On the other hand, the orientation of $\left\{D_{\boldsymbol{u}} \mathcal{W}\right\}_{\boldsymbol{u} \in \mathcal{B}(\underline{\underline{\phi}}, \underline{\kappa})}$ only depends on the orientation on $W_{\kappa_{i}}^{u}$. As we vary $\theta$, the critical point $\kappa_{i}=\left(p, x_{i}\right)$ is changed to $e^{\mathbf{i} \theta} \kappa_{i}:=\left(e^{-\mathbf{i} r \theta} p, e^{\mathbf{i} \theta} x_{i}\right)$. Moreover, $e^{\mathbf{i} \theta}$ will map the unstable manifold of $\kappa_{i}$ to that of $e^{\mathbf{i} \theta} \kappa_{i}$. It is also easy to see that as $\theta$ moves from 0 to $2 \pi$, the orientation on the unstable manifold doesn't alter. Therefore, the orientation bundle is trivial over the space of $\phi$. 
3.4. Virtual cycle on $\mathcal{M}_{\underline{P}}(\underline{\kappa})$. Since $\underline{P}$ is strongly regular, $\mathcal{M}_{\underline{P}}(\underline{\kappa})=\overline{\mathcal{M}}_{\underline{P}}(\underline{\kappa})$ because there is no nontrivial solitons. Hence $\mathcal{M}_{\underline{P}}(\underline{\kappa})$ is compact with respect to the weak topology. We remark that the weak topology on $\mathcal{M}_{\underline{P}}(\underline{\kappa})$ coincides with the topology induced from $\mathcal{B}$. This is due to the common feature of $\epsilon$-regularity in elliptic theories; in our case, it was proved in [TX15, Section 4] that when the energy on a cylindrical end is sufficiently small, the energy density should decay exponentially. Hence if a sequence in $\mathcal{M}_{\underline{P}}(\underline{\kappa})$ converges in the weak topology, since there cannot be any energy escape at infinity to form a nontrivial soliton in the limit, the sequence also converges strongly. Abbreviate $\mathcal{M}_{\underline{P}}(\underline{\kappa})$ by $\mathcal{M}$. The remaining of this subsection is devoted to the construction of a virtual orbifold atlas on $\mathcal{M}$.

Since the moduli spaces we considered here are compact, it is possible to construct the virtual cycle using simpler argument, For example, one can cover the whole moduli space by a single chart. However, we provide a more general argument where the atlas has charts indexed by a partially ordered set, to illustrate how to construct virtual cycle in more general cases.

3.4.1. The local model. The first step is to construct a local chart around each point of $\mathcal{M}$, represented by certain $\boldsymbol{u} \in \mathcal{F}^{-1}(0)$. If $\Sigma$ has at least one puncture, then the $\mathcal{G}$-action on $\mathcal{A}$ is free and there is no nontrivial automorphisms of solutions to $\mathcal{F}(\boldsymbol{u})=0$. Then $\mathcal{M}=\mathcal{F}^{-1}(0)$. If $\Sigma$ has no puncture (see Remark 3.11 below), then $\boldsymbol{u}$ possibly has a nontrivial finite automorphism group $\Gamma \subset G$ and $\mathcal{M} \simeq \mathcal{F}^{-1}(0) / G$. In any case, the linearization $D_{\boldsymbol{u}} \mathcal{F}$ is $\Gamma$-equivariant. By compactness of $\mathcal{F}^{-1}(0)$, there are only finitely many different automorphism groups. Let $\tilde{\Gamma} \subset G$ be the finite group generated by these automorphisms groups.

We would like to find an obstruction space $\tilde{E}_{\boldsymbol{u}} \subset \mathcal{E}_{\boldsymbol{u}}$, which is a $\Gamma_{\boldsymbol{u}}$-invariant finite dimensional subspace such that

$$
\operatorname{Image}\left(D_{\boldsymbol{u}} \mathcal{F}\right)+\tilde{E}_{\boldsymbol{u}}=\mathcal{E}_{\boldsymbol{u}}
$$

In the approach of [FO99], if such an obstruction space is chosen, then one can extend it to nearby objects. In the approach of [LT98b], we can choose the obstruction space in a more global fashion so that it can be extended to arbitrary objects (at least in the same stratum). Although there is no essential difference, but the choice will affect the remaining steps of the virtual cycle construction. In the following we will follow the approach of [LT98b].

Let $V_{c}^{\prime}$ be the space of compactly supported smooth sections of $\pi^{*} \Omega_{\Sigma^{*}}^{0,1} \otimes T^{\perp} Y$, where $\pi: Y \rightarrow \Sigma^{*}$ is the projection. Elements of $G$, viewed as constant gauge transformations, acts on $V_{c}^{\prime}$ through the gauge action on $Y$. Let $V_{c}^{\prime \prime}$ be the space of compactly supported smooth sections in $L_{\tau}^{p}(\mathfrak{g}) \oplus \overline{L_{\tau}^{p}(\mathfrak{g})}$, i.e., the target space of the vortex equation plus the gauge fixing condition.

Lemma 3.10. There exists a finite dimensional subspace $V_{\boldsymbol{u}}^{\prime} \subset V_{c}^{\prime}$ and a finite dimensional subspace $\tilde{E}_{\boldsymbol{u}}^{\prime \prime} \subset V_{c}^{\prime \prime}$ such that if we denote $\tilde{E}_{\boldsymbol{u}}^{\prime}:=u^{*}\left(V_{\boldsymbol{u}}^{\prime}\right) \subset \mathcal{E}_{\boldsymbol{u}}^{\prime}$ and $\tilde{E}_{\boldsymbol{u}}=\tilde{E}_{\boldsymbol{u}}^{\prime} \oplus \tilde{E}_{\boldsymbol{u}}^{\prime \prime}$, then (3.7) holds. Moreover, when $\Sigma$ has no punctures, $V_{\boldsymbol{u}}^{\prime}$ can be chosen to be $\tilde{\Gamma}$ invariant. 
Proof. It suffices to prove that there exists $V_{u}^{\prime} \subset V_{c}^{\prime}$ such that the partial derivative of $\mathcal{W}$ at $\boldsymbol{u}$ in the $u$-direction is transversal to $u^{*} V_{\boldsymbol{u}}^{\prime}$. This partial derivative is a CauchyRiemann type operator, which gives a Fredholm operator $D_{\boldsymbol{u}} \mathcal{W}$. Then there exists a finite dimensional subspace $\tilde{E}_{\boldsymbol{u}}^{\prime} \subset L_{\tau}^{p}\left(\Sigma^{*}, \Lambda^{0,1} \otimes u^{*} T^{\perp} Y\right)$ which is generated by sections with compact support and which is transversal to the image of $D_{\boldsymbol{u}} \mathcal{W}$. Since $u$ is embedded into the total space $Y$, one can extend $\tilde{E}_{\boldsymbol{u}}^{\prime}$ to a globally defined subspace $V_{\boldsymbol{u}}^{\prime}$ of compactly supported sections on $Y$. Lastly, when $\Sigma$ has no punctures and $\boldsymbol{u}$ may have a nontrivial automorphism group $\Gamma_{\boldsymbol{u}} \subset G$, we enlarge $V_{\boldsymbol{u}}^{\prime}$ to $\Gamma_{\boldsymbol{u}} V_{\boldsymbol{u}^{\prime}}$, which is still transverse to the image of $D_{\boldsymbol{u}} \mathcal{W}$.

For $e=\left(e^{\prime}, e^{\prime \prime}\right) \in \tilde{E}_{\boldsymbol{u}}^{\prime} \oplus \tilde{E}_{\boldsymbol{u}}^{\prime \prime}$ and $\boldsymbol{u}^{\prime}=\left(A^{\prime}, u^{\prime}, \varphi^{\prime}\right) \in \mathcal{B}$, denote $e\left(\boldsymbol{u}^{\prime}\right)=\left(e^{\prime}\left(\boldsymbol{u}^{\prime}\right), e^{\prime \prime}\right)$, where $e^{\prime}\left(\boldsymbol{u}^{\prime}\right) \in \Gamma\left(\Sigma^{*}, \Lambda^{0,1} \otimes\left(u^{\prime}\right)^{*} T^{\perp} Y\right)$ is the pullback of $e^{\prime}$ via $u^{\prime}$. Then one can consider the following section

$$
\mathcal{F}_{\boldsymbol{u}}: \tilde{E}_{\boldsymbol{u}} \times \mathcal{B} \rightarrow \mathcal{E}, \quad \mathcal{F}_{\boldsymbol{u}}\left(e, \boldsymbol{u}^{\prime}\right)=e\left(\boldsymbol{u}^{\prime}\right)+\mathcal{F}\left(\boldsymbol{u}^{\prime}\right)
$$

By the transversality condition of $E_{\boldsymbol{u}}$, there exists a $\Gamma_{\boldsymbol{u}}$-invariant neighborhood $\mathcal{U}_{\boldsymbol{u}} \subset \mathcal{B}$ of $\boldsymbol{u}$ such that $\tilde{U}_{\boldsymbol{u}}:=\mathcal{F}_{\boldsymbol{u}}^{-1}(0) \cap\left(\tilde{E}_{\boldsymbol{u}} \times \mathcal{U}_{\boldsymbol{u}}\right)$ is a smooth manifold. We shrink $\mathcal{U}_{\boldsymbol{u}}$ such that

$$
\mathcal{U}_{\boldsymbol{u}} \cap \gamma \mathcal{U}_{\boldsymbol{u}}=\varnothing, \forall \gamma \in \tilde{\Gamma} \backslash \Gamma_{\boldsymbol{u}} .
$$

Denote $U_{\boldsymbol{u}}=\tilde{U}_{\boldsymbol{u}} / \Gamma_{\boldsymbol{u}}$ which is an orbifold. By abuse of notation, denote $E_{\boldsymbol{u}}=\left(\tilde{U}_{\boldsymbol{u}} \times\right.$ $\left.\tilde{E}_{\boldsymbol{u}}\right) / \Gamma_{\boldsymbol{u}}$ as an orbibundle over $U_{\boldsymbol{u}}$. There is the natural section $S_{\boldsymbol{u}}: U_{\boldsymbol{u}} \rightarrow E_{\boldsymbol{u}}$ induced by the projection $\tilde{\mathcal{U}} \times \tilde{E}_{\boldsymbol{u}} \rightarrow \tilde{E}_{\boldsymbol{u}} . S_{\boldsymbol{u}}^{-1}(0)$ naturally embeds into $\mathcal{M}_{\underline{P}}(B)$, via a map $\psi_{\boldsymbol{u}}$ whose image $F_{\boldsymbol{u}}$ is an open neighborhood of $\boldsymbol{u}$ in $\mathcal{M}_{\underline{P}}(B)$. This gives a local chart $C_{\boldsymbol{u}}=\left(U_{\boldsymbol{u}}, E_{\boldsymbol{u}}, S_{\boldsymbol{u}}, \psi_{\boldsymbol{u}}, F_{\boldsymbol{u}}\right)$.

3.4.2. The local charts. Choose finitely many $\boldsymbol{u}_{i} \in \mathcal{M}, i=1, \ldots, N$ such that the collection of footprints $\left\{F_{\boldsymbol{u}_{i}}\right\}$ in the charts constructed above forms an open cover of $\mathcal{M}$. Now we construct a collection of charts $C_{i}=\left(U_{i}, E_{i}, S_{i}, \psi_{i}, F_{i}\right)$ still indexed by elements in $\{1, \ldots, N\}$ such that there are coordinate changes among them. For each $i$, define

$$
\Gamma_{i}:=\left\langle\Gamma_{\boldsymbol{u}_{1}}, \ldots, \Gamma \boldsymbol{u}_{i}\right\rangle \subset \tilde{\Gamma} \subset G, \quad \tilde{E}_{i}:=\bigoplus_{1 \leq l \leq i} \tilde{E}_{\boldsymbol{u}_{i}} .
$$

An element of $\tilde{E}_{i}$ is denoted by $\underline{e}_{i}=\left(e_{1}, \ldots, e_{i}\right)$. Since each $\tilde{E}_{\boldsymbol{u}_{i}}$ is $\tilde{\Gamma}$-invariant, $\tilde{E}_{i}$ is acted by $\Gamma_{i}$. Moreover, for $i \leq j$, there is a natural inclusion $\tilde{E}_{i} \hookrightarrow \tilde{E}_{j}$.

On the other hand, define the section

$$
\mathcal{F}_{i}: \tilde{E}_{i} \times \mathcal{B} \rightarrow \mathcal{E}, \quad \mathcal{F}_{i}\left(e_{1}, \ldots, e_{i}, \boldsymbol{u}\right)=\sum_{1 \leq l \leq i} e_{l}(\boldsymbol{u})+\mathcal{F}(\boldsymbol{u}) .
$$

By the $\Gamma_{i}$-invariance of $E_{i}$ and the gauge invariance of $\mathcal{F}$, one has that $\mathcal{F}_{i}$ is a $\Gamma_{i^{-}}$ equivariant section. Denote $\mathcal{U}_{i}=\Gamma_{i} \mathcal{U}_{\boldsymbol{u}_{i}} \subset \mathcal{B}$, which is a $\Gamma_{i}$-invariant open subset of the Banach manifold. Since $\mathcal{U}_{\boldsymbol{u}_{i}} \subset \mathcal{B}$ is chosen such that $\mathcal{F}_{\boldsymbol{u}_{i}}^{-1}(0) \cap\left(\tilde{E}_{\boldsymbol{u}_{i}} \times \mathcal{U}_{\boldsymbol{u}_{i}}\right)$ is transverse, the "thickening" $\tilde{U}_{i}:=\mathcal{F}_{i}^{-1}(0) \cap\left(\tilde{E}_{i} \times \mathcal{U}_{i}\right)$ is also a $\Gamma_{i}$-invariant smooth manifold. Define $U_{i}=$ $\tilde{U}_{i} / \Gamma_{i}$ which is an orbifold (where the $\Gamma_{i}$-action is effective), and denote $E_{i}:=\left(\tilde{E}_{i} \times \tilde{U}_{i}\right) / \Gamma_{i}$ the orbifold bundle over $U_{i}$. Similar as above, one obtains a chart $C_{i}=\left(U_{i}, E_{i}, S_{i}, \psi_{i}, F_{i}\right)$. Notice that $F_{i}=F_{\boldsymbol{u}_{i}}$, so the collection of $F_{i}$ still cover $\mathcal{M}$. 
3.4.3. The coordinate changes. Now we construct coordinate changes. For each pair $i \leq j$, if $F_{i} \cap F_{j} \neq \varnothing$, define

$$
\mathcal{U}_{j i}=\mathcal{U}_{i} \cap \mathcal{U}_{j}
$$

It is $\Gamma_{i}$-invariant since $\Gamma_{i} \subset \Gamma_{j}$. Define

$$
\tilde{U}_{j i}=\mathcal{F}_{i}^{-1}(0) \cap\left(\tilde{E}_{i} \times \mathcal{U}_{j i}\right) \subset \tilde{U}_{i}, \quad U_{j i}=\tilde{U}_{j i} / \Gamma_{i} .
$$

Then $U_{j i} \subset U_{i}$ is open and the footprint is exactly $F_{i} \cap F_{j}$. Moreover, because $\tilde{E}_{i} \subset \tilde{E}_{j}$, there is an equivariant embedding $\tilde{U}_{j i} \rightarrow \tilde{U}_{j}$ which descends to an orbifold embedding $\phi_{j i}: U_{j i} \rightarrow U_{j}$. Combining with the inclusion $\tilde{E}_{i} \hookrightarrow \tilde{E}_{j}$, we obtain a bundle embedding $\widehat{\phi}_{j i}:\left.E_{i}\right|_{U_{j i}} \rightarrow E_{j}$.

Now we check that the triple $T_{j i}=\left(U_{j i}, \phi_{j i}, \widehat{\phi}_{j i}\right)$ is indeed a coordinate change from $C_{i}$ to $C_{j}$ in the sense of Definition 7.22. First, by construction, the footprint of $U_{j i}$ is $F_{i} \cap F_{j}$. Second, if $x_{k} \in U_{i}$ converges to $x_{\infty} \in U_{i}$ and the embedding images $y_{k}=\phi_{j i}\left(x_{k}\right)$ converges to $y_{\infty} \in U_{j}$, then we can lift $x_{k}, y_{k}$ to sequences $\tilde{x}_{k}=\tilde{y}_{k} \in \mathcal{U}_{j i}$. The convergences imply that $\tilde{x}_{k}$ and $\tilde{y}_{k}$ converge to $\tilde{x}_{\infty}=\tilde{y}_{\infty} \in \mathcal{U}_{j i}$. Hence $x_{\infty} \in U_{j i}$ and $y_{\infty}=\phi_{j i}\left(x_{\infty}\right)$. Therefore, $T_{j i}$ is a coordinate change from $C_{i}$ to $C_{j}$.

We check the cocycle condition and the overlapping condition of Definition 7.24. The cocycle condition follows directly from our construction. The overlapping condition holds because the index set $\{1, \ldots, N\}$ is totally ordered. Therefore, the atlas $\mathfrak{A}=$ $\left(\left\{C_{i}\right\},\left\{T_{j i}\right\}\right)$ is a virtual orbifold atlas. By Theorem 7.28, an appropriate shrinking of $\mathfrak{A}$ produces a good atlas.

Lastly, combining the previous discussion on orientations, the good atlas is oriented. Then by the abstract topological construction of the virtual cycle given in the section7, there is a well-defined virtual cardinality $\# \mathcal{M} \in \mathbb{Q}$ whenever the virtual dimension is zero. This finishes the proof of Theorem 3.1.

Remark 3.11. We comment on a special case. When there is no puncture, i.e., there is a smooth $r$-th root of $K_{\Sigma}$, which is only possible when $r$ divides $2 g-2$, the $\mathcal{G}_{\tau}$-action on $\mathcal{B}$ may not be free because $\mathcal{G}_{\tau}$ contains constant gauge transformations now. On the other hand, by the energy identity, if $(A, u)$ is a solution in this case, then $u(\Sigma) \subset$ Crit $W$ and $(A, u)$ is also a solution to the symplectic vortex equation. In this case, in order to get a good theory, one has to choose the moment map (by adding to $\mu$ a constant in $\mathfrak{g}$ ) so that for solutions $(A, u), u(\Sigma)$ is not contained in $\mu^{-1}(c)$ for a singular value $c$ of $\mu$. Then the automorphism group of a solution $(A, u)$ is at most finite.

Remark 3.12. There have been various choices made in the construction of the good virtual orbifold atlas. We have to prove that the virtual count is independent of these choices. The two major choices are: the finite many charts $C_{\boldsymbol{u}_{i}}$ centered at $\left[\boldsymbol{u}_{i}\right] \in \mathcal{M}$ whose footprints cover $\mathcal{M}$; the various shrinkings we made in order to achieve a good virtual orbifold atlas. The independence from the second set of choices can be proved in an abstract setting. On the other hand, if we have two collections of charts $\left\{C_{\boldsymbol{u}_{i}}\right\}$ and $\left\{C_{\boldsymbol{u}_{i}^{\prime}}\right\}$, then one can construct a virtual orbifold atlas with boundary on the product $\mathcal{M} \times[0,1]$, such that the two boundary components have the corresponding virtual orbifold atlases constructed out of these two collection of basic charts. Using this cobordism one can prove that the virtual count is well-defined. 


\section{Invariance of the Correlation Function}

In this section we prove the invariance of the correlation function under changes of strongly regular perturbations. The main idea of the proof has been provided in [TX16] and we give more details here, although the most technical part (the wall-crossing formula) will be completed in the companion paper [TX].

Theorem 4.1. The correlation function defined by (3.5) is independent of the choice of the strongly regular perturbation $\underline{P}$.

We need to compare the correlation functions defined for two different strongly regular perturbations. This comparison is obviously reduced to the case that they only differ for a single broad puncture. Therefore, to save notations for the remaining of this paper, we assume that $\mathcal{C}$ has only one broad marking $\mathbf{z}$ with monodromy labelled by $m \in\{0,1, \ldots, r-1\}$. Let $\phi \in \mathbf{F r}$ be the frame variable.

The proof consists of several steps. First we reduce the comparison to the case that $a_{-}=a_{+}=a$. This reduction is obtained in the next subsection. Then to compare $\left(a, F_{-}\right)$ and $\left(a, F_{+}\right)$, we connect $F_{-}$and $F_{+}$by a smooth path $F_{\iota} \in \mathbf{V} \backslash \mathbf{V}_{a}^{\text {sing }}$ and use a cobordism argument. The path may cross the wall where the perturbation is not strongly regular, and we have to prove a wall-crossing formula (Theorem 4.6). Using the wall-crossing formula the invariance of the correlation function is proved.

4.1. Independence on $a$. Take $a \in \mathbb{C}^{*}$ and $F \in \mathbf{V} \backslash\left(\mathbf{V}_{a}^{\text {sing }} \cup \mathbf{V}_{a}^{\text {wall }}\right)$. For $a_{\lambda}=\lambda^{r} a$ with $\lambda>0$, we define $F_{\lambda}(x)=\lambda^{r} F\left(\lambda^{-1} x\right)$ where we view $\lambda$ as an element of $\left(G^{\prime}\right)^{\mathbb{C}}$. Denote $P_{\lambda}=\left(a_{\lambda}, F_{\lambda}\right)$ and $\tilde{W}_{\lambda}=W-a_{\lambda} p+F_{\lambda}$. Then

$$
\left\{\begin{array} { c } 
{ Q ( x ) = a ; } \\
{ p d Q ( x ) + d F ( x ) = 0 }
\end{array} \Longleftrightarrow \left\{\begin{array}{c}
Q(\lambda x)=a_{\lambda} ; \\
p d Q(\lambda x)+d F_{\lambda}(\lambda x)=0
\end{array}\right.\right.
$$

Then $\kappa=(p, x) \in \operatorname{Crit}\left[\left.\tilde{W}\right|_{\tilde{X}_{\gamma}}\right]$ implies that $\kappa_{\lambda}:=(p, \lambda x) \in \operatorname{Crit}\left[\left.\tilde{W}_{\lambda}\right|_{\tilde{X}_{\gamma}}\right]$. Moreover, $\tilde{W}_{\lambda}\left(\kappa_{\lambda}\right)=\lambda^{r} \tilde{W}(\kappa)$. Therefore, $P$ is strongly regular if and only if $P_{\lambda}$ is strongly regular. Now for any $\lambda>1$, consider the family of perturbations $P_{\alpha}$ for $\alpha \in[1, \lambda]$. Consider the moduli space

$$
\tilde{\mathcal{M}}_{\tilde{P}}(\kappa)=\left\{(\alpha,[\boldsymbol{u}]) \mid \alpha \in[1, \lambda],[\boldsymbol{u}] \in \mathcal{M}_{P_{\alpha}}\left(\kappa_{\alpha}\right)\right\} .
$$

Theorem 4.2. There is an oriented virtual orbifold atlas on $\tilde{\mathcal{M}}_{\tilde{P}}(\tilde{\kappa})$ with oriented virtual boundary $\left[\mathcal{M}_{P_{\lambda}}\left(\kappa_{\lambda}\right)\right] \sqcup\left[-\mathcal{M}_{P}(\kappa)\right]$. Therefore $\# \mathcal{M}_{P}(\kappa)=\# \mathcal{M}_{P_{\lambda}}\left(\kappa_{\lambda}\right)$.

Proof. The proof is a standard homotopy argument and we omit the details.

Therefore, the comparison is reduced to the case that $a_{-}=e^{\mathrm{i} \theta} a_{+}=e^{\mathrm{i} \theta} a$ for some $\theta \in[0,2 \pi)$. Take $F_{\theta}(x)=F\left(e^{-\mathbf{i} \theta / r} x\right)$ and $P_{\theta}=\left(e^{\mathbf{i} \theta} a, F_{\theta}\right)$ and $\tilde{W}_{\theta}=W-a_{\theta} p+F_{\theta}$. Then

$$
\left\{\begin{array} { c } 
{ Q ( x ) = a ; } \\
{ p d Q ( x ) + d F ( x ) = 0 }
\end{array} \Longleftrightarrow \left\{\begin{array}{c}
Q\left(e^{\mathbf{i} \theta / r} x\right)=a_{\theta} ; \\
\left(e^{-\mathbf{i} \theta} p\right) d Q\left(e^{\mathbf{i} \theta / r} x\right)+d F_{\theta}\left(e^{\mathbf{i} \theta / r} x\right)=0
\end{array}\right.\right.
$$

Then if $\kappa=(p, x)$ is a critical point of $\tilde{W}$ restricted to $\tilde{X}_{\gamma}$, then $\kappa_{\theta}:=\left(e^{-\mathbf{i} \theta} p, e^{\mathbf{i} \theta / r} x\right)$ is a critical point of $\tilde{W}_{\theta}$ restricted to $\tilde{X}_{\gamma}$. Moreover, $\tilde{W}_{\theta}\left(\kappa_{\theta}\right)=\tilde{W}(\kappa)$. So all critical values of $\tilde{W}_{\theta}$ still have distinct imaginary parts, namely, $P_{\theta}$ is strongly regular. 
Theorem 4.3. There exist a homeomorphism $\mathcal{M}_{P}(\kappa) \simeq \mathcal{M}_{P_{\theta}}\left(\kappa_{\theta}\right)$ and an identification between their oriented virtual orbifold atlases, compatible with this homeomorphism. Therefore $\# \mathcal{M}_{P}(\kappa)=\# \mathcal{M}_{P_{\theta}}\left(\kappa_{\theta}\right)$.

Proof. We define a gauge transformation $g_{\theta}^{\prime \prime}: \Sigma^{*} \rightarrow G^{\prime \prime}$ which is equal to $e^{\mathbf{i} \theta / r}$ over $C_{i}$ and, by using a cut-off function, extended to the identity away from $C_{i}$. Notice that this is not an element of $\mathcal{G}$. For any solution $\boldsymbol{u}=(A, u, \phi) \in \tilde{\mathcal{M}}_{P}(\kappa)$, define

$$
\boldsymbol{u}_{\theta}=\left(A_{\theta}, u_{\theta}, \phi_{\theta}\right):=\left(g_{\theta}^{*} A, g_{\theta}^{*} u, e^{-\mathbf{i} \theta / r} \phi\right) .
$$

We would like to show that $\boldsymbol{u}_{\theta}$ is a solution to the gauged Witten equation with perturbation $P_{\theta}$. First, away from the cylindrical end where $\tilde{\mathcal{W}}_{A, \phi}$ coincides with the unperturbed $\mathcal{W}_{A}, \boldsymbol{u}_{\theta}$ still solves the equation by the gauge invariance property of $\tilde{\mathcal{W}}_{A, \phi}$. On the cylindrical end, denote $\check{g}_{\theta}^{\prime \prime}=e^{-\mathbf{i} \theta / r} g_{\theta}^{\prime \prime}$. Then we see that $\check{A}_{\theta}:=\left(\check{g}_{\theta}^{\prime \prime}\right)^{*} A=\left(g_{\theta}^{\prime \prime}\right)^{*} A$. Then by the property of $\tilde{\mathcal{W}}_{A, \phi}$ (see Subsection 2.4), over the cylindrical end one has

$$
\tilde{\mathcal{W}}_{A_{\theta}, \phi_{\theta}}=\tilde{\mathcal{W}}_{\check{A}_{\theta}, \phi_{\theta}}=\tilde{\mathcal{W}}_{A, \phi_{\theta}} \circ \check{g}_{\theta}^{\prime \prime}=\tilde{\mathcal{W}}_{A, \phi} \circ g_{\theta}^{\prime \prime}
$$

Then since $g_{\theta}^{\prime \prime}$ is a unitary gauge transformation which preserves the metric, one has

$$
\left(g_{\theta}^{\prime \prime}\right)_{*}\left[\bar{\partial}_{A_{\theta}} u_{\theta}+\nabla \tilde{\mathcal{W}}_{A_{\theta}, \phi_{\theta}}\left(u_{\theta}\right)\right]=\bar{\partial}_{A} u+\left(g_{\theta}^{\prime \prime}\right)_{*} \nabla \tilde{\mathcal{W}}_{A_{\theta}, \phi_{\theta}}\left(u_{\theta}\right)=\bar{\partial}_{A} u+\nabla \tilde{\mathcal{W}}_{A, \phi}(u)=0 .
$$

Therefore, $\boldsymbol{u}_{\theta}$ solves the gauged Witten equation with perturbation $P_{\theta}$. Hence $g_{\theta}^{\prime \prime}$ induces a homeomorphism between $\mathcal{M}_{P}(\kappa)$ and $\mathcal{M}_{P_{\theta}}\left(\kappa_{\theta}\right)$. It also induces an identification between the the virtual orbifold atlases because $g_{\theta}^{\prime \prime}$ is globally defined.

Corollary 4.4. The correlation functions defined for $P$ and $P_{\theta}$ coincide.

Proof. The map $f_{\theta}: x \mapsto e^{\mathbf{i} \theta / r} x$ induces an isomorphism

$$
f_{\theta}^{*}: H^{\operatorname{mid}}\left(Q_{\gamma}^{a_{\theta}}\right)^{\mathbb{Z}_{r}} \simeq H^{\operatorname{mid}}\left(Q_{\gamma}^{a}\right)^{\mathbb{Z}_{r}}
$$

which intertwines with the isomorphisms $H^{\operatorname{mid}}\left(Q_{\gamma}^{a_{\theta}}\right)^{\mathbb{Z}_{r}} \simeq \mathcal{H}_{\gamma} \simeq H^{\operatorname{mid}}\left(Q_{\gamma}^{a}\right)^{\mathbb{Z}_{r}}$. Moreover $f_{\theta}$ maps gradient flows of $\left.F\right|_{Q_{\gamma}^{a}}$ to the gradient flow of $\left.F_{\theta}\right|_{Q_{\gamma}^{a}}$ since $f_{\theta}$ is an isometry. Therefore, given $\mathcal{O} \in \mathcal{H}_{\gamma} \simeq H^{\text {mid }}\left(Q_{\gamma}^{a}\right)^{\mathbb{Z}_{r}}$ which corresponds to a cocycle $\mathcal{O}_{\theta} \in H^{\text {mid }}\left(Q_{\gamma}^{a_{\theta}}\right)^{\mathbb{Z}_{r}}$, for any $\left.\kappa \in \operatorname{Crit} \tilde{W}\right|_{\tilde{X}_{\gamma}}$ and $\left.\kappa_{\theta} \in \operatorname{Crit} \tilde{W}_{\theta}\right|_{\tilde{X}_{\gamma}}$, one has

$$
\mathcal{O}^{*} \cap\left[W_{\kappa}^{u}\right]=\mathcal{O}_{\theta}^{*} \cap\left[W_{\kappa_{\theta}}^{u}\right] .
$$

By the definition of the correlation function (3.5) and Theorem 4.3, the correlation functions defined for $P$ and $P_{\theta}$ coincide.

4.2. The wall-crossing. Now we reduce the comparison between correlation functions for the two strongly regular perturbations to the case that $a_{+}=a_{-}=a$. The detail of this part will be greatly expanded and will be contained in the companion paper [TX]. Here we only discuss up to the point where the wall-crossing formula appears, and how it implies the invariance of the correlation function. Consider $F_{ \pm} \in \mathbf{V} \backslash\left(\mathbf{V}_{a}^{\text {sing }} \cup \mathbf{V}_{a}^{\text {wall }}\right)$. Since $\mathbf{V}_{a}^{\text {sing }}$ is a proper complex analytic set, $\mathbf{V} \backslash \mathbf{V}_{a}^{\text {sing }}$ is path-connected. So there is a smooth path $\tilde{F}$ in $\mathbf{V} \backslash \mathbf{V}_{a}^{\text {sing }}$ connecting $F_{ \pm}$. We also write the path $\tilde{F}$ by $F_{\iota}$ for $\iota \in\left[\iota_{-}, \iota_{+}\right]$and denote $P_{\iota}=\left(a, F_{\iota}\right)$. We can perturb the path such that it only intersects $\mathbf{V}_{a}^{\text {wall }}$ through its smooth locus transversely. Therefore we may assume that 
the intersection only appears at $\iota=\iota_{0} \in\left(\iota_{-}, \iota_{+}\right)$. By the definition of $\mathbf{V}_{a}^{\text {wall }}$, there are smooth paths $v_{\iota},\left.\left.\kappa_{\iota} \in \operatorname{Crit} \tilde{W}_{\iota}\right|_{\tilde{X}_{\gamma}} \simeq \operatorname{Crit} F_{\iota}\right|_{Q_{\gamma}^{a}}$ such that

$$
\begin{gathered}
\operatorname{Re} F_{\iota_{0}}\left(v_{\iota_{0}}\right)>\operatorname{Re} F_{\iota_{0}}\left(\kappa_{\iota_{0}}\right), \operatorname{Im} F_{\iota_{0}}\left(v_{\iota_{0}}\right)=\operatorname{Im} F_{\iota_{0}}\left(\kappa_{\iota_{0}}\right), \\
\left.\frac{d}{d \iota}\right|_{\iota=\iota_{0}}\left[\operatorname{Im} F_{\iota}\left(v_{\iota}\right)-\operatorname{Im} F_{\iota}\left(\kappa_{\iota}\right)\right] \neq 0 .
\end{gathered}
$$

The other critical values all have distinct imaginary parts for all $\iota \in\left[\iota_{-}, \iota_{+}\right]$. Denote

$$
v_{ \pm}=v_{\iota_{ \pm}}, \kappa_{ \pm}=\kappa_{\iota_{ \pm}} \text {. }
$$

Definition 4.5. We define $(-1)^{\tilde{F}}:=\operatorname{sign} \tilde{F}$ to be $+1 /-1$ if (4.1) is positive/negative.

Theorem 4.6. In the situation described above, the following holds. For $\kappa^{\prime} \neq \kappa$, one has $\# \mathcal{M}_{P_{+}}\left(\kappa^{\prime}\right)=\# \mathcal{M}_{P_{-}}\left(\kappa^{\prime}\right)$. On the other hand,

$$
\# \mathcal{M}_{P_{+}}(\kappa)-\# \mathcal{M}_{P_{-}}(\kappa)=-(-1)^{\tilde{F}}\left[\# \mathcal{N}\left(v_{\iota_{0}}, \kappa_{\iota_{0}}\right)\right]\left[\# \mathcal{M}_{P_{-}}(v)\right]
$$

Here $\# \mathcal{N}\left(v_{\iota_{0}}, \kappa_{\iota_{0}}\right)$ is the algebraic counts of the number of BPS solitons in $\tilde{X}_{\gamma}$ for the perturbed function $\tilde{W}_{\iota_{0}}=W-a p+F_{\iota_{0}}$ between the two critical points $v_{\iota_{0}}$ and $\kappa_{\iota_{0}}$.

The above theorem is referred to as the bifurcation or wall-crossing formula, which will be proved in the companion paper [TX]. Here we briefly explain the proof. One needs to construct a virtual fundamental chain over a universal moduli space fibred over $\left[\iota_{-}, \iota_{+}\right]$parametrizing the path of perturbations. Because of the appearance of soliton solutions for the non-strongly regular perturbation $P_{\iota_{0}}$, the universal moduli space is not compact. One can compactify it by adding soliton solutions. Moreover, when constructing the virtual chain, an important fact is that non-BPS soliton solutions are interior points and BPS soliton solutions are boundary points of the universal moduli space. This is why the counting of BPS solitons appears in the wall-crossing formula. This is the same situation happening in Hamiltonian Floer theory when one has timeindependent Hamiltonians and almost complex structures, and this phenomenon also appeared in [FJR] in the case of Landau-Ginzburg theory.

On the other hand, the coefficients $\mathcal{O}_{i}^{*} \cap\left[W_{\kappa_{i}}^{u}\right]$ in (3.5) also undergo a bifurcation when crossing the wall. This is just the famous Picard-Lefschetz formula, stated as follows. A key issue is the sign.

Proposition 4.7 (Picard-Lefschetz formula). For $M$ sufficiently large, we have

$$
\left[W_{v_{+}}^{u}\right]-\left[W_{v_{-}}^{u}\right]=(-1)^{\tilde{F}} \# \mathcal{S}\left(v_{\iota_{0}}, \kappa_{\iota_{0}}\right)\left[W_{\kappa_{\iota_{0}}}^{u}\right] \in H_{\text {mid }}\left(Q_{\gamma}^{a}, \quad \boldsymbol{R e} F_{\iota_{0}} \leq-M\right) .
$$

Here $\# \mathcal{S}\left(v_{\iota_{0}}, \kappa_{\iota_{0}}\right)$ is the algebraic count of BPS solitons in $Q_{\gamma}^{a}$ for the function $F_{\iota_{0}}$ between the two critical points $v_{\iota_{0}}$ and $\kappa_{\iota_{0}}$ (see Definition 6.4).

This proposition is proved in Section 6 where a key issue is to make the sign correct. A further step is to identify the two countings $\# \mathcal{N}\left(v_{\iota_{0}}, \kappa_{\iota_{0}}\right)$ and $\# \mathcal{S}\left(v_{\iota_{0}}, \kappa_{\iota_{0}}\right)$.

Proposition 4.8. One has $\# \mathcal{N}\left(v_{\iota_{0}}, \kappa_{\iota_{0}}\right)=\# \mathcal{S}\left(v_{\iota_{0}}, \kappa_{\iota_{0}}\right)$.

The proof, given in Section 5, is based on an adiabatic limit argument which was previously used in [SX14]. 
Now one can derive the equality between the correlation functions for $F_{\iota_{-}}$and $F_{\iota_{+}}$. The proof has already been given in [TX16]. However we still include it here.

For each path of critical points $\kappa_{\iota} \in \operatorname{Crit}\left[\left.\tilde{W}_{\iota}\right|_{\tilde{X}_{\gamma}}\right]$, we denote by $n_{\kappa}^{ \pm}$the virtual cardinality of the moduli space $\mathcal{M}_{P_{ \pm}}\left(\kappa_{ \pm}\right)$. Then the correlation functions for $P_{ \pm}$are linear functionals on $H^{\operatorname{mid}}\left(Q_{\gamma}^{a} ; \mathbb{Q}\right)^{\mathbb{Z}_{r}}$ defined by

$$
\langle\mathcal{O}\rangle_{ \pm}=\sum_{\kappa^{ \pm} \in \operatorname{Crit}\left[\tilde{W}_{\tilde{X}_{\gamma}}^{ \pm}\right]} n_{\kappa}^{ \pm} \cdot\left(\mathcal{O}^{*} \cap\left[\left(\kappa_{ \pm}\right)^{-}\right]\right)
$$

Suppose the wall-crossing happens between the two paths $\kappa_{\iota}^{\prime}, \kappa_{\iota}^{\prime \prime}$ such that

$$
\boldsymbol{\operatorname { R e }} \tilde{W}_{\iota}\left(\kappa_{\iota}^{\prime}\right)<\boldsymbol{\operatorname { R e }} \tilde{W}_{\iota}\left(\kappa_{\iota}^{\prime \prime}\right),\left.\frac{d}{d \iota}\right|_{\iota=0}\left[\operatorname{Im} \tilde{W}_{\iota}\left(\kappa_{\iota}^{\prime}\right)-\operatorname{Im} \tilde{W}_{\iota}\left(\kappa_{\iota}^{\prime \prime}\right)\right] \neq 0
$$

Then we have $n_{\kappa}^{+}=n_{\kappa}^{-}$for $\kappa \neq \kappa^{\prime},\left[W_{\kappa_{+}}^{u}\right]=\left[W_{\kappa_{-}}^{u}\right]$ for $\kappa \neq \kappa^{\prime \prime}$. Moreover, by Theorem 4.6, Proposition 4.7 and Proposition 4.8, one has

$$
\begin{gathered}
\langle\mathcal{O}\rangle_{+}-\langle\mathcal{O}\rangle_{-}=n_{\kappa^{\prime}}^{+} \cdot\left(\mathcal{O}^{*} \cap\left[W_{\kappa_{+}^{\prime}}^{u}\right]\right)+n_{\kappa^{\prime \prime}}^{+} \cdot\left(\mathcal{O}^{*} \cap\left[W_{\kappa_{+}^{\prime \prime}}^{u}\right]\right)-n_{\kappa^{\prime}}^{-} \cdot\left(\mathcal{O}^{*} \cap\left[W_{\kappa_{-}^{\prime}}^{u}\right]\right)+n_{\kappa^{\prime \prime}}^{-} \cdot\left(\mathcal{O}^{*} \cap\left[W_{\kappa_{-}^{\prime \prime}}^{u}\right]\right) \\
=\left(n_{\kappa^{\prime}}^{+}-n_{\kappa^{\prime}}^{-}\right) \cdot\left(\mathcal{O}^{*} \cap\left[W_{\kappa_{+}^{\prime}}^{u}\right]\right)+n_{\kappa^{\prime \prime}}^{+} \cdot\left(\mathcal{O}^{*} \cap\left(\left[W_{\kappa_{+}^{\prime \prime}}^{u}\right]-\left[W_{\kappa_{-}^{\prime \prime}}^{u}\right]\right)\right) \\
=-(-1)^{\tilde{F}}\left(\# \mathcal{N}\left(\kappa_{0}^{\prime \prime}, \kappa_{0}^{\prime}\right)\right) \cdot n_{\kappa^{\prime \prime}}^{+} \cdot\left(\mathcal{O}^{*} \cap\left[W_{\kappa_{+}^{\prime}}^{u}\right]\right)+n_{\kappa^{\prime \prime}}^{+} \cdot\left(\mathcal{O}^{*} \cap(-1)^{\tilde{F}} \# \mathcal{N}\left(\kappa_{0}^{\prime \prime}, \kappa_{0}^{\prime}\right)\left[W_{\kappa_{-}^{\prime}}^{u}\right]\right)=0 .
\end{gathered}
$$

Therefore it completes the proof of Theorem 4.1.

\section{Proof of Proposition 4.8}

In this section we proof Proposition 4.8. Since we will work purely inside the fixed point $\tilde{X}_{\gamma} \subset \tilde{X}$, to save notations we assume that $\gamma=$ Id. Before going into details let us look at the problem from an intuitive perspective. Let $F=F_{\iota_{0}} \in \mathbf{V}_{a} \backslash \mathbf{V}_{a}^{\text {sing }}$ be the regular but not strongly regular perturbation. Denote $F^{a}:=\left.F\right|_{Q^{a}}$. Denote $\tilde{W}=W-a p+F$. Choose $v, \kappa \in \operatorname{Crit} \tilde{W}$, which, by the principle of Lagrange multiplier, corresponds to two critical points of the restriction $F^{a}$. By abuse of notation we still denote them by $v$ and $\kappa$. Let $\mathcal{S}_{F^{a}}(v, \kappa)$ be the space of solutions to

$$
\frac{d y}{d s}+\nabla F^{a}(y(s))=0, \lim _{s \rightarrow-\infty} y(s)=v, \lim _{s \rightarrow+\infty} y(s)=\kappa
$$

modulo time translation. On the other hand, the gradient flow equation of $\tilde{W}$ can be written in components as

$$
\left\{\begin{aligned}
\frac{d x}{d s}+\overline{p(s)} \nabla Q(x(s))+\nabla F(x(s)) & =0, \\
\frac{d p}{d s}+\overline{Q(x(s))-a} & =0 .
\end{aligned}\right.
$$


We introduce a real parameter $\lambda>0$ and consider

$$
\left\{\begin{aligned}
\frac{d x}{d s}+\overline{p(s)} \nabla Q(x(s))+\nabla F(x(s)) & =0, \\
\frac{d p}{d s}+\lambda^{2}[\overline{Q(x(s))-a}] & =0, \\
\lim _{s \rightarrow-\infty}(x(s), p(s)) & =v, \\
\lim _{s \rightarrow+\infty}(x(s), p(s)) & =\kappa .
\end{aligned}\right.
$$

In finite dimensional Morse theory, it is convenient to consider the energy functional

$$
E(x, p)=\frac{1}{2}\left[\|\overline{p(s)} \nabla Q(x)+\nabla F(x)\|_{L^{2}}^{2}+\lambda^{2}\|\overline{Q(x)-a}\|_{L^{2}}^{2}+\|\dot{x}\|_{L^{2}}^{2}+\frac{1}{\lambda^{2}}\|\dot{p}\|_{L^{2}}^{2}\right] .
$$

The energy of a solution to (5.1) is equal to $\tilde{W}(v)-\tilde{W}(\kappa)$, which is independent of $\lambda$. As indicated by the result of [SX14], as $\lambda \rightarrow+\infty$, solutions are approximately negative gradient lines of the restriction $\mathbf{R e} F^{a}$. If we denote by $\mathcal{S}_{\tilde{W}}^{\lambda}(v, \kappa)$ the moduli space of solutions to (5.1) (modulo translation), then we will construct an orientation-preserving homeomorphism

$$
\mathcal{S}_{\tilde{W}}^{\lambda}(v, \kappa) \simeq \mathcal{S}_{F^{a}}(v, \kappa), \forall \lambda>>0
$$

Notice that $\mathcal{S}_{\tilde{W}}^{1}(v, \kappa)=\mathcal{N}(v, \kappa)$. Moreover, by a homotopy argument the (virtual) counting of $\mathcal{S}_{\tilde{W}}^{\lambda}(v, \kappa)$ is independent of $\lambda$. This will complete the proof of Proposition 4.8. In the remaining of this section, we carry out the details of the above arguments.

5.1. On transversality. Generically, there is no flow line connecting two distinct critical points with equal index. On the other hand, for a holomorphic Morse function $f$ on an $n$-dimensional complex manifold, critical points of $f$, viewed as critical points of $\operatorname{Re} f$, all have index $n$. Therefore, a BPS soliton, viewed as a negative gradient flow line of $\boldsymbol{R e} f$, is not transverse. For any solution $\rho: \mathbb{R} \rightarrow X$ to the equation

$$
\rho^{\prime}(s)+\nabla f(\rho(s))=0, \lim _{s \rightarrow-\infty} \rho(s)=p, \lim _{s \rightarrow+\infty} \rho(s)=q,
$$

its linearization $D_{\rho}: W^{1,2}\left(x^{\star} T X\right) \rightarrow L^{2}\left(x^{\star} T X\right)$ is not transverse; a necessary condition for having one solution is $\operatorname{Im} f(p)=\operatorname{Im} f(q)$.

We introduce the equation on pairs $\hat{\rho}=(h, \rho)$ where $h \in \mathbb{R}$ and $\rho: \mathbb{R} \rightarrow X$,

$$
\rho^{\prime}(s)+\nabla f(\rho(s))-h J \nabla f(\rho(s))=0, \lim _{s \rightarrow-\infty} \rho(s)=p, \lim _{s \rightarrow+\infty} \rho(s)=q .
$$

If $(h, \rho)$ solves the solution, then $\rho$ is a negative gradient flow line of $\boldsymbol{R e}(f+h \mathbf{i} f)$, hence

$$
\operatorname{Im}(f(p)+h \mathbf{i} f(p))=\operatorname{Im}(f(q)+h \mathbf{i} f(q)),
$$

which implies that $h=0$. Therefore, solutions to (5.3) and solutions to (5.4) are in one-to-one correspondence, by identifying $\rho$ with $\hat{\rho}=(0, \rho)$.

Definition 5.1. A solution $\rho$ to (5.3) is called maximally transverse (with respect to the Hermitian metric) if the corresponding linearization $\hat{D}_{\hat{\rho}}$ of $(5.4)$ is surjective, or equivalent, $\operatorname{Coker} D_{\rho}$ is one-dimensional (spanned by $J \nabla f(\rho)$ ). 
Now we specialize to the equation (5.1). Consider the Banach manifold $\mathcal{B}$ of $W_{\text {loc }}^{1,2}$ maps from $\mathbb{R}$ to $\tilde{X}$ which are asymptotic to $v$ and $\kappa$ at $+\infty$ and $-\infty$ respectively, both in a $W^{1,2}$-sense. Consider the bundle $\mathcal{E} \rightarrow \mathcal{B}$ whose fibre over $\rho=\left(x_{\rho}, p_{\rho}\right) \in \mathcal{B}$ is

$$
\mathcal{E}_{\rho}=L^{2}\left(\rho^{*} T \tilde{X}\right)=L^{2}\left(x_{\rho}^{*} T X \oplus \mathbb{C}\right)
$$

There is a family of sections

$$
\mathcal{F}_{\tilde{W}}^{\lambda}(\rho)=\left[\begin{array}{c}
\dot{x}_{\rho}+\bar{p}_{\rho} \nabla Q\left(x_{\rho}\right)+\nabla F\left(x_{\rho}\right) \\
\dot{p}_{\rho}+\lambda^{2}\left[\overline{\left(Q\left(x_{\rho}\right)-a\right)}\right]
\end{array}\right] .
$$

Its linearization at $\rho$ reads

$$
D_{\rho}^{\lambda}(V, h)=\left[\begin{array}{c}
\nabla_{t} V+\nabla_{V}(\bar{p} \nabla Q+\nabla F)+\bar{h} \nabla Q \\
h^{\prime}+\lambda^{2} d Q \cdot V
\end{array}\right] .
$$

Denote

$$
\begin{array}{ll}
\tilde{\mathcal{S}}_{\tilde{W}}^{\lambda}(v, \kappa):=\left(\mathcal{F}_{\tilde{W}}^{\lambda}\right)^{-1}(0), & \mathcal{S}_{\tilde{W}}^{\lambda}(v, \kappa)=\tilde{\mathcal{S}}_{\tilde{W}}^{\lambda}(v, \kappa) / \mathbb{R} ; \\
\tilde{\mathcal{S}}_{\tilde{W}}:=\bigsqcup_{\lambda \geq 1}\{\lambda\} \times \tilde{\mathcal{S}}_{\tilde{W}}^{\lambda}(v, \kappa), & \mathcal{S}_{\tilde{W}}:=\bigsqcup_{\lambda \geq 1}\{\lambda\} \times \mathcal{S}_{\tilde{W}}^{\lambda}(v, \kappa) .
\end{array}
$$

$\tilde{\mathcal{S}}_{\tilde{W}}$ can be viewed as the zero locus of $\mathcal{F}_{\tilde{W}}:[1,+\infty) \times \mathcal{B} \rightarrow \mathcal{E}$ with $\mathcal{F}_{\tilde{W}}(\lambda, \rho)=\mathcal{F}_{\tilde{W}}^{\lambda}(\rho)$ It is not hard to show that by perturbing the Hermitian metric on $X$, one can assume the following:

(1) For any $\tilde{\rho}:=(\lambda, \rho) \in \tilde{\mathcal{S}}_{\tilde{W}}$, the linearization of $\mathcal{F}_{\tilde{W}}$ at $\tilde{\rho}$ has one-dimensional cokernel.

(2) Each $\rho \in \tilde{\mathcal{S}}_{\tilde{W}}^{1}$ is maximally transverse.

(3) Each $y \in \tilde{\mathcal{S}}_{F^{a}}(v, \kappa)$ is maximally transverse w.r.t. the induced metric on $Q^{a}$.

5.2. Adiabatic limit. We first need to establish the compactness of $\tilde{\mathcal{S}}_{\tilde{W}}$ as $\lambda \rightarrow+\infty$. Recall that $\star \in X$ is the unique critical point of $Q$. Therefore there is a well-defined function $q: X \backslash\{\star\} \rightarrow \mathbb{C}$ defined by

$$
[q(x) d Q(x)+d F(x)] \cdot \nabla Q(x)=0 .
$$

Notice that for $x \in Q^{a}$,

$$
\overline{q(x)} \nabla Q+\nabla F(x)=\nabla F^{a}(x) \in T_{x} Q^{a} .
$$

Proposition 5.2. Let $\lambda_{i}$ be a sequence of positive numbers such that $\lim \lambda_{i}=+\infty$. Suppose $\tilde{x}_{i}(s)=\left(x_{i}(s), p_{i}(s)\right)$ is a sequence of solutions to (5.1) with $\lambda=\lambda_{i}$. Then there is a subsequence (still indexed by $i$ ), a sequence of numbers $s_{i} \in \mathbb{R}$, and a solution to

$$
\dot{x}(s)+\nabla F^{a}(x(s))=0, x: \mathbb{R} \rightarrow Q^{a}, \lim _{s \rightarrow-\infty} x(s)=v, \lim _{s \rightarrow+\infty} x(s)=\kappa
$$

such that the following conditions hold.

(1) $x_{i}\left(s+s_{i}\right)$ converges to $x(s)$ uniformly on compact subsets of $\mathbb{R}$.

(2) $p_{i}\left(s+s_{i}\right)$ converges to $q(x(s))$ uniformly. 
Proof. The key point is the $C^{0}$-bound, i.e., there exists a compact subset $\tilde{K} \subset \tilde{X}$ such that $\tilde{x}_{i}(s) \in \tilde{K}$. Once this is proved, this proposition can be proved in the same way as in [SX14]. Let $3 d_{a}=\operatorname{dist}\left(\star, Q^{a}\right)>0$. First, we claim that for $i$ large enough, $x_{i}(s) \notin B_{d_{a}}(\star)$ for all $s \in \mathbb{R}$. We prove this claim by contradiction. Suppose $d\left(\star, x_{i}\left(s_{i}\right)\right) \leq d_{a}$. Let $\left[s_{i}-a_{i}, s_{i}+b_{i}\right]$ be the maximal interval containing $s_{i}$ such that $x_{i}\left(\left[s_{i}-a_{i}, s_{i}+b_{i}\right]\right) \subset B_{2 d_{a}}(\star)$. Then we have

$$
d_{a} \leq \operatorname{dist}\left(x_{i}\left(s_{i}-a_{i}\right), x_{i}\left(s_{i}\right)\right) \leq \int_{s_{i}-a_{i}}^{s_{i}}\left|\dot{x}_{i}(s)\right| d s \leq \sqrt{a_{i}}\left\|\dot{x}_{i}\right\|_{L^{2}} \leq \sqrt{a_{i} E} .
$$

Here $E$ is the energy of the solution $\tilde{x}_{i}$ defined by (5.2). Therefore, $a_{i} \geq d_{a}^{2} / E$. Similarly $b_{i} \geq d_{a}^{2} / E$. On the other hand, there is $\epsilon_{a}>0$ such that $\left|Q\left(x_{i}(s)\right)-a\right| \geq \epsilon_{a}$ for all $s \in\left[s_{i}-a_{i}, s_{i}+b_{i}\right]$. Then

$$
E \geq \lambda_{i}^{2}\left\|Q\left(x_{i}\right)-a_{i}\right\|_{L^{2}(\mathbb{R})}^{2} \geq \lambda_{i}^{2}\left\|Q\left(x_{i}\right)-a_{i}\right\|_{L^{2}\left(\left[s_{i}-a_{i}, s_{i}+b_{i}\right]\right)}^{2} \geq \lambda_{i}^{2} \epsilon_{a}^{2} d_{a}^{2} / E .
$$

This is impossible for $i$ sufficiently large. Therefore the claim is proved.

Second, by the definition of $q(x)$, the properness of $\nabla Q$ (see Hypothesis 2.1) and the boundedness of $\nabla F$ (see Hypothesis 2.5), it is easy to see that $q$ and $\nabla q$ are uniformly bounded away from $B_{d_{a}}(\star)$. By (5.1), we have

$$
\begin{aligned}
0 & =\frac{d}{d s} Q\left(x_{i}\right)-d Q\left(x_{i}\right) \cdot \dot{x}_{i} \\
& =\frac{d}{d s} Q\left(x_{i}\right)+d Q \cdot\left[\overline{p_{i}} \nabla Q\left(x_{i}\right)+\nabla F\left(x_{i}\right)\right] \\
& =\frac{d}{d s} Q\left(x_{i}\right)+d Q \cdot\left[\overline{\left(p_{i}-q\left(x_{i}\right)\right)} \nabla Q\left(x_{i}\right)\right] \\
& =\frac{d}{d s} Q\left(x_{i}\right)+\lambda_{i}\left|\nabla Q\left(x_{i}\right)\right|^{2}\left[\lambda_{i}^{-1} \overline{\left(p_{i}-q\left(x_{i}\right)\right)}\right] .
\end{aligned}
$$

We also have

$$
\begin{aligned}
& \frac{d}{d s}\left[\lambda_{i}^{-1}\left(p_{i}-q\left(x_{i}\right)\right)\right]+\lambda_{i}\left[Q\left(x_{i}\right)-a\right] \\
& =\lambda_{i}^{-1}\left[\dot{p}_{i}-d q \cdot \dot{x}_{i}\right]+\lambda_{i}\left[Q\left(x_{i}\right)-a\right] \\
& =-\lambda_{i}^{-1} d q \cdot \dot{x}_{i} .
\end{aligned}
$$

Consider the operator

$$
\begin{aligned}
D_{\lambda_{i}}: & W^{1,2}\left(\mathbb{R}, \mathbb{C}^{2}\right) \rightarrow L^{2}\left(\mathbb{R}, \mathbb{C}^{2}\right) \\
& \left(f_{1}, f_{2}\right) \mapsto\left(\frac{d f_{1}}{d s}+\lambda_{i}\left|\nabla Q\left(x_{i}\right)\right|^{2} \overline{f_{2}}, \frac{d f_{2}}{d s}+\lambda_{i} f_{1}\right) .
\end{aligned}
$$

Notice that $\left|\nabla Q\left(x_{i}\right)\right|$ is uniformly bounded from below. Then as an unbounded operator from $L^{2}$ to $L^{2}, D_{\lambda_{i}}$ is bounded from below by $c \lambda_{i}$ for some constant $c>0$. On the other hand, it has a right inverse from $L^{2}$ to $W^{1,2}$ which is uniformly bounded. Hence by (5.5), (5.6), we have

$$
\begin{gathered}
\left\|Q\left(x_{i}\right)-a\right\|_{L^{2}(\mathbb{R})}+\lambda_{i}^{-1}\left\|p_{i}-q\left(x_{i}\right)\right\|_{L^{2}(\mathbb{R})} \leq c \lambda_{i}^{-2}\left\|d q \cdot \dot{x}_{i}\right\|_{L^{2}(\mathbb{R})} \leq c \lambda_{i}^{-2}\left\|\dot{x}_{i}\right\|_{L^{2}(\mathbb{R})} . \\
\left\|Q\left(x_{i}\right)-a\right\|_{W^{1,2}(\mathbb{R})}+\lambda_{i}^{-1}\left\|p_{i}-q\left(x_{i}\right)\right\|_{W^{1,2}(\mathbb{R})} \leq c \lambda_{i}^{-1}\left\|\dot{x}_{i}\right\|_{L^{2}(\mathbb{R})} .
\end{gathered}
$$

In particular, $\left\|p_{i}\right\|_{L^{\infty}}$ and $\left\|\dot{p}_{i}\right\|_{L^{2}}$ are uniformly bounded. 
Now we prove that $x_{i}(s)$ is uniformly bounded. Consider the moment map $\mu^{\prime}: X \rightarrow$ $i \mathbb{R}$ of the $G^{\prime}$-action. For some constant $c>0$, we have

$$
\begin{aligned}
\frac{d^{2}}{d s^{2}} \mathbf{i} \mu^{\prime}\left(x_{i}\right) & =\partial_{s}\left\langle\nabla \mathbf{i} \mu^{\prime}\left(x_{i}\right), \dot{x}_{i}\right\rangle \\
& =\partial_{s}\left\langle\nabla \mathbf{i} \mu^{\prime}\left(x_{i}\right),-\overline{p_{i}} \nabla Q\left(x_{i}\right)-\nabla F\left(x_{i}\right)\right\rangle \\
& =-\partial_{s}\left[\operatorname{Re}\left(r W\left(\tilde{x}_{i}\right)+F_{b}\left(x_{i}\right)\right)\right] \\
& =-\partial_{s}\left[\operatorname{Re}\left(r \tilde{W}\left(\tilde{x}_{i}\right)+F_{b}\left(x_{i}\right)-r F\left(x_{i}\right)-r a p_{i}\right)\right] \\
& =-r \nabla^{\lambda_{i}} \tilde{W}\left(\tilde{x}_{i}\right) \cdot \dot{\tilde{x}}_{i}-\nabla\left(F_{b}-r F\right) \cdot \dot{x}_{i}+r a \mathbf{R e}\left(\dot{p}_{i}\right) \\
& \geq r\left|\dot{\tilde{x}}_{i}\right|_{\lambda_{i}}^{2}-c\left|\dot{x}_{i}\right|-c\left|\dot{p}_{i}\right| \\
& \geq-c\left|\dot{x}_{i}\right|-c\left|\dot{p}_{i}\right| .
\end{aligned}
$$

Here $F_{b}=\left\langle\nabla\left(\mathbf{i} \mu^{\prime}\right), \nabla F\right\rangle$ and the second last inequality follows from (1) of Hypothesis 2.5 , i.e., $F$ behaves like a linear function. Notice that $\dot{x}_{i}$ and $\dot{p}_{i}$ are both bounded in $L^{2}$. If there exists $s_{i} \in \mathbb{R}$ such that

$$
\lim _{i \rightarrow \infty} \mathbf{i} \mu^{\prime}\left(x_{i}\left(s_{i}\right)\right)=\lim _{i \rightarrow+\infty} \sup _{\mathbb{R}} \mathbf{i} \mu^{\prime}\left(x_{i}\right)=+\infty,
$$

then it is easy to derive that $\mathbf{i} \mu^{\prime}\left(x_{i}\left(\cdot+s_{i}\right)\right)$ diverges to infinity uniformly on compact sets. However, if this is true, then by (3) of Hypothesis 2.5 (which says $\nabla \tilde{W}$ is uniformly bounded from below outside a compact subset), the energy of $\tilde{x}_{i}$ can be infinitely large, which is impossible. Hence $\mathbf{i} \mu^{\prime}\left(x_{i}\right)$ is uniformly bounded. Since $\mu^{\prime}$ is proper (see Hypothesis 2.1), $x_{i}$ is uniformly bounded.

It follows from (5.7) that $x_{i}$ is in a small neighborhood of $Q^{a}$. Therefore, one can write $x_{i}(s)=\left(\bar{x}_{i}(s), Q\left(x_{i}(s)\right)\right)$ with $\bar{x}_{i}(s) \in Q^{a}$. Moreover, for some $c>0$,

$$
\left|\dot{\bar{x}}_{i}(s)+\nabla F_{a}\left(\bar{x}_{i}(s)\right)\right|=\left|\dot{\bar{x}}_{i}(s)+q\left(\bar{x}_{i}(s)\right) \nabla Q\left(\bar{x}_{i}(s)\right)+\nabla F\left(\bar{x}_{i}(s)\right)\right| \leq c\left|Q\left(x_{i}(s)\right)-a\right|
$$

which converges uniformly to zero. Hence a subsequence of $\bar{x}_{i}$ (still indexed by $i$ ) converges (modulo reparametrization) to a flow line of $F_{a}$. Moreover, the flow line cannot break because it must connect $v$ and $\kappa$. This finishes the proof of Proposition 5.2 .

Now we define a compactification of $\mathcal{S}_{\tilde{W}}$, i.e.,

$$
\overline{\mathcal{S}_{\tilde{W}}}:=\mathcal{S}_{\tilde{W}} \sqcup\left[\{+\infty\} \times \mathcal{S}_{F^{a}}(v, \kappa)\right] .
$$

We say that a sequence $\left[\tilde{\rho}_{i}\right]=\left[\lambda_{i}, \rho_{i}\right] \in \mathcal{S}_{\tilde{W}}$ converges to $[+\infty, y(s)]$ if up to translation, the two conditions of Proposition 5.2 hold.

Lastly, we need construct a boundary chart near the $+\infty$ side of $\overline{\mathcal{S}_{\tilde{W}}}$. We have

Proposition 5.3. Suppose the negative gradient line $y: \mathbb{R} \rightarrow Q^{a}$ is maximally transverse. Then there exist $\Lambda=\Lambda_{y}>0$, and a continuous map

$$
\Phi_{y}:[\Lambda,+\infty] \rightarrow \overline{\mathcal{S}_{\tilde{W}}}
$$

which is a homeomorphism onto a neighborhood of $[+\infty, y(s)]$. 
This proposition will be proved shortly. It puts a boundary chart on $\overline{\mathcal{S}_{\tilde{W}}}$ and hence implies an oriented cobordism from $\mathcal{N}(v, \kappa)$ to $\mathcal{S}_{F^{a}}(v, \kappa)$. Therefore,

$$
\# \mathcal{N}(v, \kappa)(v, \kappa)=\# \mathcal{S}_{F^{a}}(v, \kappa) .
$$

This finishes the proof of Proposition 4.8.

5.3. Proof of Proposition 5.3. Choose a small $\epsilon>0$. Let $Q^{a, \epsilon}$ be the $\epsilon$-neighborhood of $Q^{a}$ inside $X$. Let $\left.L \subset T X\right|_{Q^{a, \epsilon}}$ be the rank two bundle spanned by $\nabla Q$ and $J \nabla Q$; let $L^{\perp}$ be its orthogonal complement. Then for those $\rho \in \mathcal{B}$ which are contained in $Q^{a, \epsilon}$, we can decompose the domain and the target space of the linearization $D_{\rho}^{\lambda}$ as

$$
\begin{aligned}
\mathcal{T}_{\rho} \mathcal{B} & \simeq W_{L}(\rho) \oplus W_{T}(\rho)=W^{1,2}\left(x^{*} L \oplus \mathbb{C}\right) \oplus W^{1,2}\left(x^{\star} L^{\perp}\right), \\
\mathcal{E}_{\rho} \simeq \mathcal{E}_{L}(\rho) \oplus \mathcal{E}_{T}(\rho) & =L^{2}\left(x^{*} L \oplus \mathbb{C}\right) \oplus L^{2}\left(x^{\star} L^{\perp}\right) .
\end{aligned}
$$

We rescale the norms on $W_{L}(\rho)$ and $\mathcal{E}_{L}(\rho)$ as follows. We identify $\left(h_{1} \nabla Q, h_{2}\right) \in W_{L}(\rho)$ with $\left(h_{1}, h_{2}\right) \in W^{1,2} \oplus W^{1,2}$ and define

$$
\left\|\left(h_{1}, h_{2}\right)\right\|_{W_{\lambda}}=\lambda\left\|h_{1}\right\|_{L^{2}}+\left\|h_{1}^{\prime}\right\|_{L^{2}}+\left\|h_{2}\right\|_{L^{2}}+\lambda^{-1}\left\|h_{2}^{\prime}\right\|_{L^{2}} .
$$

For $\left(h_{1} \nabla Q, h_{2}\right) \in \mathcal{E}_{L}(\rho)$, we define

$$
\left\|\left(h_{1}, h_{2}\right)\right\|_{L_{\lambda}}=\left\|h_{1}\right\|_{L^{2}}+\lambda^{-1}\left\|h_{2}\right\|_{L^{2}} .
$$

The norms on the tangential components are unchanged, and we use $W_{\lambda}$ and $L_{\lambda}$ to denote the modified norms on $T_{\rho} \mathcal{B}$ and $\mathcal{E}_{\rho}$ respectively.

Let $y: \mathbb{R} \rightarrow Q^{a}$ be a negative gradient line of $F^{a}$. Then the linearization along $y$ reads

$$
D_{y}: W^{1,2}\left(y^{*} T Q^{a}\right) \rightarrow L^{2}\left(y^{*} T Q^{a}\right), D_{y}\left(\xi_{y}\right)=\nabla_{s} \xi_{y}+\nabla_{\xi_{y}}(\nabla F+\overline{q(y)} \nabla Q) .
$$

We define

$$
D_{y}^{+}: \mathbb{R} \oplus W^{1,2}\left(y^{*} T Q^{a}\right) \rightarrow L^{2}\left(y^{*} T Q^{a}\right), D_{y}^{+}\left(a, \xi_{y}\right)=D_{y}\left(\xi_{y}\right)-a J(\nabla F+\overline{q(y)} \nabla Q) .
$$

Since $y$ is maximally transverse, $D_{y}^{+}$is surjective.

Now we define $\rho_{y} \in \mathcal{B}$ by $\rho_{y}(s)=(y(s), q(y(s)))$. This is going to be our approximate solution to (5.1) for $\lambda$ large. To apply the implicit function theorem, we have a few estimates to make. First, by straightforward calculation,

$$
\left\|\mathcal{S}^{\lambda}\left(\rho_{y}\right)\right\|_{L_{\lambda}}=\lambda^{-1}\left\|d q \cdot y^{\prime}\right\|_{L^{2}} .
$$

Let $D_{\rho_{y}}^{\lambda}: \mathcal{T}_{\rho_{y}} \mathcal{B} \rightarrow \mathcal{E}_{\rho_{y}}$ be the linearization along $\rho_{y}$. For $\xi_{y} \in W_{T}\left(\rho_{y}\right) \subset \mathcal{T}_{\rho_{y}} \mathcal{B}$, we have

$$
D_{\rho_{y}}^{\lambda}\left(\xi_{y}\right)=\left(\nabla_{t} \xi_{y}+\nabla_{\xi_{y}}(\nabla F+\overline{q(y)} \nabla Q), \lambda^{2} d Q \cdot \xi_{y}\right)=\left(D_{y}\left(\xi_{y}\right), 0\right) .
$$

Hence with respect to the decompositions in $(5.9), D_{\rho_{y}}$ can be written as

$$
D_{\rho_{y}}^{\lambda}=\left[\begin{array}{cc}
D_{y} & A_{y} \\
0 & D_{y}^{\prime}
\end{array}\right]
$$

where for $H=\left(h_{1} \nabla Q, h_{2}\right) \in W_{L}\left(\rho_{y}\right), A_{y}(H)$ is real linear in $h_{1}$. Therefore, by (5.10) (5.11),

$$
\left\|A_{y}\left(h_{1} \nabla Q, h_{2}\right)\right\|_{L_{\lambda}} \leq c_{1}\left\|h_{1}\right\|_{L^{2}} \leq c_{1} \lambda^{-1}\|H\|_{W_{\lambda}}
$$


On the other hand, it is easy to see that $D_{y}^{\prime}: W_{L}\left(\rho_{y}\right) \rightarrow \mathcal{E}_{L}\left(\rho_{y}\right)$ has an inverse $Q_{y}^{\prime}$ whose operator norm with respect to the modified Banach norms (5.10) and (5.11) is bounded by a number independent of $\lambda$. Therefore, we construct the operator

$$
Q_{\rho_{y}}^{+}=\left[\begin{array}{cc}
Q_{y}^{+} & 0 \\
0 & Q_{y}^{\prime}
\end{array}\right]:\left(\mathbb{R} \oplus \mathcal{E}_{T}\left(\rho_{y}\right)\right) \oplus \mathcal{E}_{T}\left(\rho_{y}\right) \rightarrow W_{T}\left(\rho_{y}\right) \oplus W_{L}\left(\rho_{y}\right) .
$$

Moreover, for certain constant $c>0$,

$$
\left\|\operatorname{Id}-D_{\rho_{y}}^{+} Q_{\rho_{y}}^{+}\right\|_{\lambda}=\left\|Q_{y}^{+} A_{y}\right\|_{\lambda} \leq c \lambda^{-1} .
$$

Therefore, for $\lambda$ sufficiently large, $Q_{\rho_{y}}^{+}$is approximately a right inverse to $D_{\rho_{y}}^{+}$.

Thirdly, we need to estimate the variation of the linearized operator near $\rho_{y}$. One can give a local chart of the Banach manifold $\mathcal{B}$ near $\rho_{y}$ and trivialize the bundle $\mathcal{E} \rightarrow \mathcal{B}$ over this chart as follows. Using the identification $Q^{a, \epsilon} \simeq Q^{a} \times B_{\epsilon}$, for any $\xi=\left(\xi_{y}, h_{1} \nabla Q, h_{2}\right) \epsilon$ $\mathcal{T}_{\rho_{y}} \mathcal{B}$ with small norm, we identify it with the map $\Phi^{B}(\xi):=\left(\overline{\exp }_{y} \xi_{y}, h_{1}, q(y)+h_{2}\right)$ into $Q^{a} \times B_{\epsilon} \times \mathbb{C}$, where $\overline{\exp }$ is the exponential map inside $Q^{a}$. We trivialize $\mathcal{E}$ over the image of $\Phi^{B}$. Let $D_{\xi}^{\lambda,+}: \mathbb{R} \oplus \mathcal{T}_{\rho_{y}} \mathcal{B} \rightarrow \mathcal{E}_{\rho_{y}}$ be the linearization of $\Phi^{E} \circ \mathcal{F}_{\tilde{W}}^{\lambda,+} \circ \Phi^{B}$ at $\xi \in \mathcal{T}_{\rho_{y}} \mathcal{B}$.

Lemma 5.4. There exist, $\epsilon, c, \Lambda>0$ such that for $\lambda \geq \Lambda$ and $\|\xi\|_{W_{\lambda}} \leq \epsilon$, we have

$$
\left\|D_{\xi}^{\lambda,+}-D_{\rho_{y}}^{\lambda,+}\right\| \leq c\|\xi\|_{W_{\lambda}} .
$$

Proof. Similar to [SX14, Lemma 9] and the detail is left to the reader.

Therefore, one can apply the implicit function theorem and one derives that for each sufficiently large $\lambda$, there is a unique $\xi_{\lambda}^{+}=\left(a_{\lambda}, \xi_{\lambda}\right) \in \operatorname{Image}\left(Q_{\rho_{y}}^{+}\right) \subset \mathbb{R} \oplus \mathcal{T}_{\rho_{y}} \mathcal{B}$ such that

$$
\left(a_{\lambda}, \exp _{\rho_{y}} \xi_{\lambda}\right) \in\left(\mathcal{F}_{\tilde{W}}^{\lambda,+}\right)^{-1}(0) .
$$

We also know that $a_{\lambda}=0$. Hence it defines a map $\Psi_{y}^{+}:[\Lambda,+\infty] \rightarrow \overline{\mathcal{S}_{\tilde{W}}}$ by $\lambda \mapsto\left[\lambda, \Phi^{B}\left(\xi_{\lambda}\right)\right]$. Moreover, one can show that it is a homeomorphism onto a neighborhood of $[+\infty, y(s)]$ inside $\overline{\mathcal{S}_{\tilde{W}}}$. The details are left to the reader. Hence $\Psi_{y}^{+}$provides a boundary chart on $\overline{\mathcal{S}_{\tilde{W}}}$ and we have finished the proof of Proposition 5.3.

\section{Proof of Proposition 4.7}

In this section we prove the Picard-Lefschetz formula (Proposition 4.7). We think it is necessary to have a detailed discussion of orientations, which determines the sign of the bifurcation term appearing in the Picard-Lefschetz formula and the wall-crossing formula (Theorem 4.6). Our discussion will still be used in the companion paper [TX].

6.1. Linear algebra. First we need to declare our rule of orienting operators. Let $F: X \rightarrow Y$ be a Fredholm operator between Banach spaces $X, Y$. The determinant line of $F$ is defined as the real one-dimensional vector space

$$
\operatorname{det} F:=\operatorname{det} \operatorname{Ker} F \otimes(\operatorname{det} \operatorname{Coker} F)^{\vee} \text {. }
$$

An orientation of $F$ is a homotopy class of trivializations of this space.

More generally, for any continuous family of Fredholm operators $\mathcal{F}=\left(F_{a}\right)_{a \in A}$ where $A$ is a topological space, there is a determinant line bundle over $A$. If $A$ is contractible, then $\operatorname{det} \mathcal{F}$ is trivial and a trivialization $\varphi_{A}: \operatorname{det} \mathcal{F} \rightarrow A \times \mathbb{R}$ can be easily write down if 
$\operatorname{dim}\left(\operatorname{Ker} F_{a}\right)$ is a constant for $a \in A$. In this case, we say $\theta_{1} \in \operatorname{det} F_{a_{1}}$ and $\theta_{2} \in \operatorname{det} F_{a_{2}}$ are in the same orientation in $\operatorname{det} \mathcal{F}$ if the second factors of $\varphi_{A}\left(\theta_{1}\right), \varphi_{A}\left(\theta_{2}\right)$ are of the same sign.

It becomes complicated when the dimensions of the kernels jump. For a Fredholm operator $F: X \rightarrow Y$, let $F_{0}^{(k)}: \mathbb{R}^{k} \oplus X \rightarrow \mathbb{R}^{k} \oplus Y$ be the operator $F_{0}^{(k)}(v, x)=(0, F(x))$. We define $\psi_{0}^{(k)}: \operatorname{det} F \rightarrow \operatorname{det} F_{0}^{(k)}$ by

$$
\psi_{0}^{(k)}\left(\bigwedge_{i=1}^{m} x_{i} \wedge \bigwedge_{j=1}^{n} y_{j}^{*}\right)=\left(\bigwedge_{l=1}^{k}\left(e_{l}, 0\right) \wedge \bigwedge_{i=1}^{m}\left(0, x_{i}\right)\right) \otimes\left(\bigwedge_{l=1}^{k}\left(e^{l}, 0\right) \wedge \bigwedge_{j=1}^{n}\left(0, y_{j}^{*}\right)\right)
$$

Here $x_{1}, \ldots, x_{m}$ is a basis of $\operatorname{Ker} F, y_{1}, \ldots, y_{n}$ is a basis of $\operatorname{Coker} F$ and $y_{1}^{*}, \ldots, y_{n}^{*}$ is the dual basis; $e_{1}, \ldots, e_{k}$ is a basis of $\mathbb{R}^{k}$ and $e^{1}, \ldots, e^{k}$ is its dual basis. We also define $F_{1}^{(k)}(v, x)=(v, F(x))$ and define $\psi_{1}^{(k)}: \operatorname{det} F \rightarrow \operatorname{det} F_{1}^{(k)}$ by

$$
\psi_{1}^{(k)}\left(\bigwedge_{i=1}^{m} x_{i} \wedge \bigwedge_{j=1}^{n} y_{j}^{*}\right)=\bigwedge_{i=1}^{m}\left(0, x_{i}\right) \otimes \bigwedge_{j=1}^{n}\left(0, y_{j}^{*}\right)
$$

The basic convention is that we regard the orientation of $\theta \in \operatorname{det} F$ as "the same" as those of $\psi_{0}^{(k)}(\theta) \in F_{0}^{(k)}$ and $\psi_{1}^{(k)}(\theta) \in \operatorname{det} F_{1}^{(k)}$.

Further, let $\mathcal{G}^{(k)}(X, Y)$ be the space of linear maps $G: \mathbb{R}^{k} \oplus X \rightarrow \mathbb{R}^{k} \oplus Y$ of the form

$$
G=\left[\begin{array}{cc}
G_{1} & G_{2} \\
G_{3} & 0
\end{array}\right]
$$

Then let $F_{G}: \mathbb{R}^{k} \oplus X \rightarrow \mathbb{R}^{k} \oplus Y$ be $F_{0}^{(k)}+G$. This gives a family of Fredholm operators over the contractible space $\mathcal{G}^{(k)}(X, Y)$ and $\operatorname{det} F_{G}$ is trivial over $\mathcal{G}^{(k)}(X, Y)$. The $F_{0}^{(k)}$ and $F_{1}^{(k)}$ above are obtained in this way by special elements in $\mathcal{G}^{(k)}(X, Y)$

$$
G_{0}^{(k)}=0, G_{1}^{(k)}=\left[\begin{array}{cc}
I_{k} & 0 \\
0 & 0
\end{array}\right]
$$

Given a continuous family of Fredholm operators $F_{T}: X \rightarrow Y, T \in[0,1]$ such that

$$
k=\operatorname{dim}\left(\operatorname{Ker} F_{0}\right)-\operatorname{dim}\left(\operatorname{Ker} F_{1}\right)=\max \left\{\operatorname{dim}\left(\operatorname{Ker} F_{T_{1}}\right)-\operatorname{dim}\left(\operatorname{Ker} F_{T_{2}}\right) \mid 0 \leq T_{1}, T_{2}\right\},
$$

we can extend the family to a family

$$
F_{T, G}: \mathbb{R}^{k} \oplus X \rightarrow \mathbb{R}^{k} \oplus Y, T \in[0,1], G \in \mathcal{G}^{(k)}(X, Y) .
$$

Two nonzero elements $\theta_{1} \in \operatorname{det} F_{1}$ and $\theta_{0} \in \operatorname{det} F_{0}$ are called in the same orientation if there is a curve $G:[0,1] \rightarrow \mathcal{G}^{(k)}(X, Y)$ such that the following conditions hold.

(1) $G(0)=G_{1}^{(k)}, G(1)=G_{0}^{(k)}$ and $\operatorname{dim}\left(\operatorname{Ker} F_{T, G(T)}\right)$ is a constant.

(2) For the family $\mathcal{F}=\left\{F_{T, G(T)} \mid T \in[0,1]\right\}$, the elements $\psi_{1}^{(k)}\left(\theta_{0}\right) \in \operatorname{det} F_{0, G(0)}$ and $\psi_{0}^{(k)}\left(\theta_{1}\right) \in \operatorname{det} F_{1, G(1)}$ are in the same orientation in $\operatorname{det} \mathcal{F}$.

With in this section, we denote $\theta_{1} \sim \theta_{2}$ if they are in the same orientation.

We treat the following special case under the above convention.

Lemma 6.1. Let $F_{T}: X \rightarrow Y, T \geq 0$ be a family of Fredholm operators of the form $F_{T}=F+T P$ where $P: X \rightarrow Y$ is of rank one. Assume the following. 
(1) $\left\{v_{1}, \ldots, v_{m}, u\right\}$ is a basis of $\mathbf{K e r} F_{0}$, and $\left\{w_{1}, \ldots, w_{n}, P(u)\right\}$ represents a basis of Coker $F_{0}$, with dual basis $\left\{w_{1}^{*}, \ldots, w_{n}^{*}, P(u)^{*}\right\}$.

(2) For all $T>0,\left\{v_{1}, \ldots, v_{m}\right\}$ is a basis of $\mathbf{K e r} F_{T},\left\{w_{1}, \ldots, w_{n}\right\} \subset Y$ represents a basis of $\operatorname{Coker} F_{T}$, and $\left\{w_{1}^{*}, \ldots, w_{n}^{*}\right\}$ is the dual basis of $\left(\operatorname{Coker} F_{T}\right)^{\vee}$.

Denote

$$
\begin{aligned}
& \theta_{T}:=\bigwedge_{i=1}^{m} v_{i} \otimes \bigwedge_{j=1}^{n} w_{i}^{*} \in \operatorname{det} F_{T},(T>0) ; \\
& \theta_{0}:=\left(u \wedge \bigwedge_{i=1}^{m} v_{i}\right) \otimes\left(P(u)^{*} \wedge \bigwedge_{j=1}^{n} w_{i}^{*}\right) \in \operatorname{det} F_{0} .
\end{aligned}
$$

Then $\theta_{1}$ and $\theta_{0}$ are in the same orientation.

Proof. Without loss of generality, assume that $n=0$. Namely, $F$ has one-dimensional cokernel spanned by $P(u)$. Define $G_{1, T}: \mathbb{R} \rightarrow \mathbb{R}, G_{2, T}: X \rightarrow \mathbb{R}$ and $G_{3, T}: \mathbb{R} \rightarrow Y$ by

$$
G_{1, T}(a)=(1-T) a, \quad G_{2, T}(x)=P(u)^{*} F_{T}(x), \quad G_{3, T}(a)=(1-T) a P(u) .
$$

Define $F_{T, G(T)}:=F_{T}+G_{1, T}+G_{2, T}+G_{3, T}$. We can check that its kernel is spanned by $\{(T e,-(1-T) u)\} \cup\left\{\left(0, v_{i}\right) \mid i=1, \ldots, m\right\}$ and its cokernel is spanned by $\left(e^{*},-P(u)^{*}\right)$, where $e \in \mathbb{R}$ is the standard basis and $e^{*}$ is its dual basis. Then

$$
\theta_{T, G(T)}:=\left[(T e,-(1-T) u) \wedge \bigwedge_{i=1}^{m}\left(0, v_{i}\right)\right] \otimes\left[\left(e^{*},-P(u)^{*}\right)\right] \in \operatorname{det} F_{T, G(T)}, T \in[0,1] .
$$

is a nowhere-vanishing section of $\operatorname{det} F_{T, G(T)}$. Furthermore, notice that

$$
F_{1, G(1)}=\left[\begin{array}{cc}
0 & P(u)^{\star} P \\
0 & F+P
\end{array}\right]
$$

can be connected by deforming the upper-right corner to $F_{0}^{(k)}$ without changing the dimension of the kernel and the element $\theta_{1, G(1)}$ has the same sign as

$$
\psi_{0}^{(k)}\left(\theta_{1}\right)=\left[e \wedge \bigwedge_{i=1}^{m}\left(0, v_{i}\right)\right] \otimes\left[\left(e^{*}, 0\right)\right] \in \operatorname{det} F_{1, G(1)}
$$

and

$$
F_{0, G(0)}=\left[\begin{array}{cc}
1 & 0 \\
P(u) & F
\end{array}\right]
$$

can be connected to $F_{1}^{(k)}$ by deforming the lower-left corner without changing the dimension of the kernel and the element $\theta_{0, G(0)}$ has the same sign as

$$
\psi_{1}^{(k)}\left(\theta_{0}\right)=\left[(0, u) \wedge \bigwedge_{i=1}^{m}\left(0, v_{i}\right)\right] \otimes\left[\left(0, P(u)^{*}\right)\right] \in \operatorname{det} F_{0, G(0)}
$$

Then by definition, $\theta_{1}$ and $\theta_{0}$ are in the same orientation. 
6.2. Orientation of BPS solitons and Picard-Lefschetz formula. We will prove the Picard-Lefschetz formula in a more general situation. Let $X$ be a Hermitian manifold and $F: X \rightarrow \mathbb{C}$ be a holomorphic Morse function. For $v \in \operatorname{Crit} F$, its unstable manifold $W_{v}^{u}$ can be identified with the solution space of the ODE

$$
\dot{x}(s)+\nabla F(x(s))=0, s \in(-\infty, 0], \lim _{s \rightarrow-\infty} x(s)=v .
$$

It is an $N$-dimensional smooth manifold homeomorphic to an $N$-disk. Choosing an orientation on $W_{v}^{u}$ is the same as choosing, for each solution $\sigma_{-}(s)$ of (6.1), an orientation of the operator

$$
D_{\sigma_{-}}: W^{1, p}\left((-\infty, 0], \sigma_{-}^{*} T X\right) \rightarrow L^{p}\left((-\infty, 0], \sigma_{-}^{*} T X\right), D_{\sigma_{-}}(\xi)=\nabla_{s} \xi+\nabla^{2} F\left(\sigma_{-}\right) \cdot \xi .
$$

Here $p \geq 2$ and the orientation of $D_{\sigma_{-}}$is consistent among all choices of Sobolev norms. Similarly, consider $\kappa \in \operatorname{Crit} F$ and its stable manifold $W_{\kappa}^{s}$. It is the solution space of

$$
\dot{x}(s)+\nabla F(x(s))=0, s \in[0,+\infty), \lim _{s \rightarrow+\infty} x(s)=\kappa .
$$

An orientation on $W_{\kappa}^{s}$ is the same as an orientation of the linearized operator of each solution $\sigma_{+}$to (6.2), which reads

$$
D_{\sigma_{+}}: W^{1, p}\left([0,+\infty), \sigma_{+}^{*} T X\right) \rightarrow L^{p}\left([0,+\infty), \sigma_{+}^{*} T X\right), D_{\sigma_{+}}=\nabla_{s} \xi+\nabla^{2} F\left(\sigma_{+}\right) \cdot \xi .
$$

Suppose $v$ and $\kappa$ are nondegenerate, and $\operatorname{Im} F(v)=\operatorname{Im} F(\kappa), \operatorname{Re} F(v)>\operatorname{Re} F(\kappa)$. Then the equation for BPS soliton connecting $v$ and $\kappa$ is

$$
\dot{x}(s)+\nabla F(x(s))=0, s \in(-\infty,+\infty), \lim _{s \rightarrow-\infty} x(s)=v, \lim _{s \rightarrow+\infty} x(s)=\kappa .
$$

Let $\tilde{\mathcal{S}}(v, \kappa)$ be the space of solutions and $\mathcal{S}(v, \kappa)=\tilde{\mathcal{S}}(v, \kappa) / \mathbb{R}$. By choosing orientations on $W_{v}^{u}$ and $W_{\kappa}^{s}$ (independently), one obtains an orientation of the linearization $D_{\sigma}$ along any solution $\sigma(s)$ as follows. Let $\sigma_{ \pm}=\left.\sigma\right|_{\mathbb{R}_{ \pm}}$. Consider the family of operators

$$
\begin{gathered}
\tilde{D}_{\sigma}^{T}: W^{1, p}\left((-\infty, 0], \sigma_{-}^{*} T X\right) \oplus W^{1, p}\left([0,+\infty), \sigma_{+}^{*} T X\right) \rightarrow L^{p}\left(\mathbb{R}, \sigma^{*} T X\right) \oplus T_{\sigma(0)} X \\
\tilde{D}_{\sigma}^{T}\left(\xi_{-}, \xi_{+}\right)=\left(D_{\sigma_{-}} \xi_{-}+D_{\sigma_{+}} \xi_{+}, T\left(\xi_{-}(0)-\xi_{+}(0)\right)\right) .
\end{gathered}
$$

Lemma 6.2. For any $T>0$, there are canonical isomorphisms

$$
\begin{gathered}
\operatorname{Ker} \tilde{D}_{\sigma}^{T} \simeq \operatorname{Ker} D_{\sigma}, \\
\operatorname{Coker}\left(\tilde{D}_{\sigma}^{T}\right)^{\vee} \simeq \operatorname{Image}\left(\tilde{D}_{\sigma}^{T}\right)^{\perp} \simeq \operatorname{Image}\left(D_{\sigma}\right)^{\perp} \simeq \operatorname{Coker}\left(D_{\sigma}\right)^{\vee} .
\end{gathered}
$$

Proof. For $T>0,\left(\xi_{-}, \xi_{+}\right) \in \operatorname{Ker} \tilde{D}_{\sigma}^{T}$ if and only if $\xi_{-}$and $\xi_{+}$are the restrictions of some $\xi \in W^{1, p}\left(\mathbb{R}, \sigma^{\star} T X\right)$. This gives the isomorphism $\operatorname{Ker} \tilde{D}_{\sigma}^{T} \simeq \operatorname{Ker} D_{\sigma}$. Moreover, if $\eta \in \operatorname{Image}\left(D_{\sigma}\right)^{\perp}$, then for $\xi_{-} \in W^{1, p}\left((-\infty, 0], \sigma_{-}^{*} T X\right)$ and $\xi_{+} \in W^{1, p}\left([0,+\infty), \sigma_{+}^{*} T X\right)$,

$$
\left\langle D_{\sigma_{ \pm}} \xi_{ \pm}, \eta\right\rangle=\mp\left\langle\xi_{ \pm}(0), \eta(0)\right\rangle \text {. }
$$

Therefore $\eta \mapsto(T \eta,-\eta(0))$ induces an isomorphism $\operatorname{Image}\left(D_{\sigma}\right)^{\perp} \simeq \operatorname{Image}\left(\tilde{D}_{\sigma}^{T}\right)^{\perp}$.

Since $T_{\sigma(0)} X$ is canonically oriented by the complex structure, Lemma 6.2 induces the following chain of isomorphisms

$$
\begin{aligned}
\operatorname{det} D_{\sigma} \simeq \operatorname{det} \tilde{D}_{\sigma}^{T} \simeq \operatorname{det} \tilde{D}_{\sigma}^{0} \simeq \operatorname{det}\left(D_{\sigma_{-}}, D_{\sigma_{+}}\right) \simeq \operatorname{det}\left(\mathbf{K e r} D_{\sigma_{-}} \oplus \mathbf{K e r} D_{\sigma_{+}}\right) \\
\simeq \operatorname{det} \mathbf{K e r} D_{\sigma_{-}} \otimes \operatorname{det} \mathbf{K e r} D_{\sigma_{+}} \simeq \operatorname{det} D_{\sigma_{-}} \otimes \operatorname{det} D_{\sigma_{+}} .
\end{aligned}
$$


Remark 6.3. If we regard $\sigma$ as a map from $\mathbb{R} \times S^{1}$ which is independent of the second coordinate, then an orientation of $D_{\sigma}$ also induces an orientation of the linearized operator over the cylinder. By the result of [FH93], this means choosing orientations on the unstable and stable manifolds induces the so-called coherent orientations on the moduli space of solitons.

It is convenient to assume that $X$ is Kähler and $\sigma$ is maximally transverse. This assumption doesn't affect the generality when discussing orientations. Then for each $\sigma \in \tilde{\mathcal{S}}(v, \kappa), J \dot{\sigma}$ spans Image $\left(D_{\sigma}\right)^{\perp}$ and we have a distinguished element

$$
\dot{\sigma} \otimes(J \dot{\sigma}) \in \operatorname{det} D_{\sigma}
$$

Definition 6.4. For $\sigma \in \tilde{\mathcal{S}}(v, \kappa)$, define $\operatorname{Sign}(\sigma) \in\{ \pm 1\}$ to be the sign of $\dot{\sigma} \otimes(J \dot{\sigma})$ with respect to the orientation of $D_{\sigma}$ induced by (6.5). This makes $\mathcal{S}(v, \kappa)$ an oriented zero-dimensional manifold. Define

$$
\# \mathcal{S}(v, \kappa)=\sum_{[\sigma] \in \mathcal{S}(v, \kappa)} \operatorname{Sign}(\sigma) .
$$

Now consider a smooth family of holomorphic functions $F_{\iota}: X \rightarrow \mathbb{C}$ with $\iota \in\left[\iota_{-}, \iota_{+}\right]$. Let the critical points of $F_{\iota}$ be $\kappa_{k, \iota} \in \operatorname{Crit} F_{\iota}$. We make the following assumptions.

(1) For each $\iota, F_{\iota}$ is Morse.

(2) For $\iota \neq \iota_{0} \in\left(\iota_{-}, \iota_{+}\right), F_{\iota}$ is strongly regular, i.e., the imaginary parts $\operatorname{Im} F_{\iota}\left(\beta_{k, \iota}\right)$ for all $k$ are distinct.

(3) At $\iota=\iota_{0}$, there are two critical points $v_{\iota_{0}}, \kappa_{\iota_{0}} \in \operatorname{Crit} F_{\iota_{0}}$ such that

$$
\begin{gathered}
\operatorname{Im} F_{\iota_{0}}\left(v_{\iota_{0}}\right)=\operatorname{Im} F_{\iota_{0}}\left(\kappa_{\iota_{0}}\right), \operatorname{Re} F_{\iota_{0}}\left(v_{\iota_{0}}\right)>\operatorname{Re} F_{\iota_{0}}\left(\kappa_{\iota_{0}}\right), \\
\left.\frac{d}{d \iota}\right|_{\iota=\iota_{0}}\left[\operatorname{Im} F_{\iota}\left(v_{\iota}\right)-\operatorname{Im} F_{\iota}\left(\kappa_{\iota}\right)\right] \neq 0 .
\end{gathered}
$$

One can identify the unstable manifold of $v_{\iota}$ with the space of solutions to the ODE

$$
\dot{x}(s)+\nabla F_{\iota}(x(s))=0, \lim _{s \rightarrow-\infty} x(s)=v_{\iota}, s \in(-\infty, 0] .
$$

We choose an orientations of $W_{v_{\iota}}^{u}$ that depends continuously on $\iota$. Then

$$
W_{v}^{u}=\bigsqcup_{\iota-\leq \iota \leq \iota_{+}} W_{v_{\iota}}^{u}
$$

has an induced orientation. It is not compact because the flow lines can break at $\iota=\iota_{0}$. One can compactify it by adding broken ones. Denote the compactification by $\overline{W_{v}^{u}}$.

Proposition 6.5. Suppose the moduli space of BPS solitons connecting $v_{\iota_{0}}$ and $\kappa_{\iota_{0}}$ is regular. Then the closure $\overline{W_{v}^{u}}$ is an oriented manifold with boundary and the boundary is the disjoint union

$$
\left[W_{v_{\iota_{+}}}^{u}\right] \sqcup\left[-W_{v_{\iota_{-}}}^{u}\right] \sqcup\left[-(-1)^{\tilde{F}} \mathcal{S}\left(v_{\iota_{0}}, \kappa_{\iota_{0}}\right) \times W_{\kappa_{\iota_{0}}}^{u}\right] .
$$

Here $\mathcal{S}\left(v_{\iota_{0}}, \kappa_{\iota_{0}}\right)$ and $W_{\kappa_{\iota_{0}}}^{u}$ have their own orientations.

Notice that $\mathcal{S}\left(v_{\iota_{0}}, \kappa_{\iota_{0}}\right)$ is zero-dimensional. Hence this proposition implies the PicardLefschetz formula (Proposition 4.7). Proposition 6.5 is proved in the next subsection. 
6.3. Proof of Proposition 6.5. Consider a Banach manifold $\tilde{\mathcal{B}}$ of pairs $(\iota, \psi)$ with

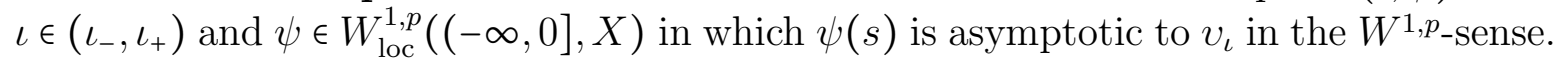
Consider the Banach bundle $\tilde{\mathcal{E}} \rightarrow \tilde{\mathcal{B}}$ whose fibre over $\tilde{\psi}=(\iota, \psi)$ is $L^{p}\left((-\infty, 0], \psi^{*} T X\right)$. Let $\tilde{\mathcal{F}}: \tilde{\mathcal{B}} \rightarrow \tilde{\mathcal{E}}$ be the section $\tilde{\mathcal{F}}(\iota, \psi)=\dot{\psi}+\nabla F_{\iota}(\psi)$. Then $W_{v}^{u} \simeq \tilde{\mathcal{F}}^{-1}(0)$ and the orientation on $W_{v}^{u}$ is given by the orientation on the linearized operator

$$
\tilde{D}_{\iota, \psi}: \mathbb{R} \times W^{1, p}\left(\mathbb{R}, \psi^{*} T X\right) \rightarrow L^{p}\left(\mathbb{R}, \psi^{*} T X\right) .
$$

We need to construct a local chart near any singular flow line. Let $\sigma \in \tilde{\mathcal{S}}\left(v_{\iota_{0}}, \kappa_{\iota_{0}}\right)$ and $b \in W_{\kappa_{\iota_{0}}}^{u}$. For $\iota-\iota_{0}$ sufficiently small, there are families of vectors

$$
\eta_{-}^{\iota} \in T_{v_{\iota_{0}}} X, \eta_{+}^{\iota} \in T_{\kappa_{\iota_{0}}} X
$$

such that

$$
\exp _{v_{\iota}} \eta_{-}^{\iota}=v_{\iota}, \exp _{\kappa_{\iota}} \eta_{+}^{\iota}=\kappa_{\iota}
$$

For $S>0$ sufficiently large, we can extend $\eta_{ \pm}^{\iota}$ to vector fields

$$
\tilde{\eta}_{-}^{\iota} \in \Gamma\left((-\infty,-S], \sigma^{*} T X\right), \tilde{\eta}_{+}^{\iota} \in \Gamma\left([S,+\infty), \sigma^{*} T X\right),
$$

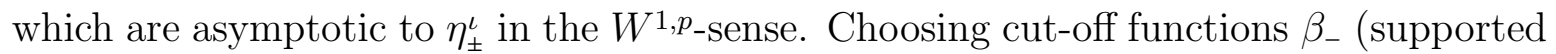
on $(-\infty,-S+1])$ and $\beta_{+}$(supported in $\left.[S-1,+\infty)\right)$, define

$$
\tilde{\eta}^{\iota}=\beta_{-} \tilde{\eta}_{-}^{\iota}+\beta_{+} \tilde{\eta}_{+}^{\iota}
$$

Denote

$$
\tilde{\eta}_{ \pm}=\left.\frac{d}{d \iota}\right|_{\iota=\iota_{0}} \tilde{\eta}_{ \pm}^{\iota}, \tilde{\eta}=\left.\frac{d}{d \iota}\right|_{\iota=\iota_{0}} \tilde{\eta}^{\iota}
$$

Then we have the linearized operator

$$
\begin{gathered}
\tilde{D}_{\sigma}: \mathbb{R}\left\{\partial_{\iota}\right\} \times W^{1, p}\left(\mathbb{R}, \sigma^{*} T X\right) \rightarrow L^{p}\left(\mathbb{R}, \sigma^{*} T X\right), \\
\tilde{D}_{\sigma}\left(a \partial_{\iota}, \xi\right)=D_{\sigma}(\xi)+L_{\sigma}\left(a \partial_{\iota}\right)
\end{gathered}
$$

where $L_{\sigma}\left(a \partial_{\iota}\right)=D_{\sigma}(a \tilde{\eta})$. Since we assumed that $\sigma$ is maximally transverse, it is easy to see that $\tilde{D}_{\sigma}$ is surjective.

We can perform the gluing construction in the standard way. The following lemma is left to the reader.

Lemma 6.6. Given a singular trajectory represented by $\sigma \in \tilde{\mathcal{S}}\left(v_{\iota_{0}}, \kappa_{\iota_{0}}\right)$ and $b \in W_{\kappa_{\iota_{0}}}^{u}$, there exists $\epsilon>0$, such that for each $t \in(0, \epsilon)$ and $\sigma^{\prime} \in B_{\epsilon}\left(\sigma, \tilde{\mathcal{S}}\left(v_{\iota_{0}}, \kappa_{\iota_{0}}\right)\right), b^{\prime} \in B_{\epsilon}\left(b, W_{\kappa_{\iota_{0}}}^{u}\right)$, there is a solution

$$
\tilde{\psi}_{t, \sigma^{\prime}, b^{\prime}}=\left(\iota_{t, \sigma^{\prime}, b^{\prime}}, \psi_{t, \sigma^{\prime}, b^{\prime}}\right) \in \tilde{\mathcal{F}}^{-1}(0) .
$$

Moreover, this family satisfies the following conditions.

(1) The map $\Phi:(0, \epsilon) \times\{\sigma\} \times B_{\epsilon}\left(b, W_{\kappa_{0}}^{u}\right) \rightarrow W_{v}^{u}$ defined by

$$
\Phi\left(t, \sigma, b^{\prime}\right):=\tilde{\psi}_{t, \sigma, b^{\prime}}
$$

extends continuously as $t \rightarrow 0$ to $\left(\sigma, b^{\prime}\right)$ and the extension

$$
\Phi:[0, \epsilon) \times\{\sigma\} \times B_{\epsilon}\left(b, W_{\kappa_{0}}^{u}\right) \rightarrow \overline{W_{v}^{u}}
$$

is a homeomorphism onto a neighborhood of the singular object $(\sigma, b)$. 
(2) Fix $t \in(0, \epsilon)$. The map $\Psi:\{t\} \times B_{\epsilon}\left(\sigma, \tilde{\mathcal{S}}\left(v_{0}, \kappa_{0}\right)\right) \times B_{\epsilon}\left(b, W_{\kappa_{0}}^{u}\right) \rightarrow W_{v}^{u}$ defined by

$$
\Psi\left(t, \sigma^{\prime}, b^{\prime}\right) \mapsto \tilde{\psi}_{t, \sigma^{\prime}, b^{\prime}}
$$

is a homeomorphism onto a neighborhood of $\tilde{\psi}_{t, \sigma, b}$.

(3) The orientations on $W_{v}^{u}$ induced from $\Phi$ and $\Psi$ are the same.

We call the orientation on $W_{v}^{u}$ in Lemma 6.6 "induced from the boundary chart." We need to see if it is consistent with the interior orientation. Indeed, define $\tilde{D}_{\sigma}^{T}=D_{\sigma}+T L_{\sigma}$ and we have isomorphisms

$$
\operatorname{det} \tilde{D}_{\sigma} \simeq \operatorname{det} \tilde{D}_{\sigma}^{T} \simeq \operatorname{det} \tilde{D}_{\sigma}^{0} .
$$

Using Lemma 6.1 , the direction represented by $(0, \dot{\sigma}) \in \operatorname{Ker} \tilde{D}_{\sigma}$ is identified with

$$
\left(\partial_{\iota}, 0\right) \wedge(0, \dot{\sigma}) \otimes L_{\sigma}\left(\partial_{\iota}, 0\right) \sim \operatorname{Sign}\left\langle L_{\sigma}\left(\partial_{\iota}\right), J \dot{\sigma}\right\rangle\left[\left(\partial_{\iota}, 0\right) \wedge(0, \dot{\sigma}) \otimes(J \dot{\sigma})\right]=: \theta^{0} \in \operatorname{det} \tilde{D}_{\sigma}^{0} .
$$

Choose a positive volume form $\omega_{b}$ on $\operatorname{Ker} D_{b}$ and a positive volume form $\omega_{\psi}$ on $\operatorname{Ker} D_{\psi}$ for any $(\iota, \psi)$ in the image of the $\Phi$ of (6.6). Since we have the canonical identification $\operatorname{det} D_{\sigma} \otimes \operatorname{det} D_{b} \simeq \operatorname{det} D_{\psi}, \theta^{0} \wedge \omega_{b} \in \operatorname{det} \tilde{D}_{\sigma}^{0} \otimes \operatorname{det} D_{b}$ is identified canonically with

$$
\operatorname{Sign}\left\langle L_{\sigma}\left(\partial_{\iota}\right), J \dot{\sigma}\right\rangle \cdot \operatorname{Sign}(\sigma) \cdot\left[\left(\partial_{\iota}, 0\right) \otimes \omega_{\psi}\right] \in \operatorname{det} \tilde{D}_{\iota, \psi}
$$

Hence the oriented boundary of $\overline{W_{v}^{u}}$ is

$$
\partial W_{v}^{u}=W_{v_{\iota_{+}}}^{u} \sqcup\left[-W_{v_{\iota_{-}}}^{u}\right] \sqcup\left[-\operatorname{Sign}\left\langle L_{\sigma}\left(\partial_{\iota}\right), J \dot{\sigma}\right\rangle\left(\mathcal{S}\left(v_{\iota_{0}}, \kappa_{\iota_{0}}\right) \times W_{\kappa_{\iota_{0}}}^{u}\right)\right] .
$$

Lastly, we need to evaluate the sign of $\left\langle L_{\sigma}\left(\partial_{\iota}\right), J \dot{\sigma}\right\rangle$. We assume that

$$
\left.\frac{d}{d \iota}\right|_{\iota=0}\left[\operatorname{Im} F_{\iota}\left(v_{\iota}\right)-\operatorname{Im} F_{\iota}\left(\kappa_{\iota}\right)\right]>0 \Longleftrightarrow(-1)^{\tilde{F}}=+1
$$

since the other case is exactly the opposite. Then by definition,

$$
\begin{aligned}
L_{\sigma}\left(\partial_{\iota}\right) & =\nabla_{s} \tilde{\eta}+\nabla^{2} F_{\iota_{0}}(\sigma) \tilde{\eta} \\
& =\nabla_{s}\left(\beta_{-} \tilde{\eta}_{-}+\beta_{+} \tilde{\eta}_{+}\right)+\nabla^{2} F_{\iota_{0}}(\sigma)\left(\beta_{-} \tilde{\eta}_{-}+\tilde{\beta}_{+} \eta_{+}\right) \\
& =\beta_{-}^{\prime} \tilde{\eta}_{-}+\tilde{\beta}_{+}^{\prime} \eta_{+} .
\end{aligned}
$$

Moreover, we know that $J \dot{\sigma}(s)=-\nabla \operatorname{Im} F_{\iota_{0}}(\sigma(s))$. Therefore,

$$
\left\langle L_{\sigma}\left(\partial_{\iota}\right), J \dot{\sigma}\right\rangle=\left\langle\beta_{-}^{\prime} \tilde{\eta}_{-},-\nabla \mathbf{I m} F_{\iota_{0}}\right\rangle+\left\langle\beta_{+}^{\prime} \tilde{\eta}_{+},-\nabla \mathbf{I m} F_{\iota_{0}}\right\rangle>0 .
$$

\section{Topological Virtual Orbifolds and Virtual Cycles}

We recall the framework of constructing virtual fundamental cycles associated to moduli problems. Such constructions, usually called "virtual technique", has a long history since it first appeared in algebraic Gromov-Witten theory by [LT98a]. The current method is based on the topological approach of [LT98b]. 
7.1. Topological manifolds and transversality. In this subsection we review the classical theory about topological manifolds and (microbundle) transversality.

Definition 7.1. (Topological manifolds and embeddings)

(1) A topological manifold is a second countable Hausdorff space $M$ which is locally homeomorphic to an open subset of $\mathbb{R}^{n}$.

(2) A subset $S \subset M$ is a submanifold if $S$ equipped with the subspace topology is a topological manifold.

(3) A map $f: N \rightarrow M$ between two topological manifold is called a topological embedding if $f$ is a homeomorphism onto its image.

(4) A topological embedding $f: N \rightarrow M$ is called locally flat if for any $p \in f(N)$, there is a local coordinate $\varphi_{p}: U_{p} \rightarrow \mathbb{R}^{m}$ where $U_{p} \subset \mathbb{R}^{n}$ is an open neighborhood of $p$ such that

$$
\varphi_{p}\left(f(N) \cap U_{p}\right) \subset \mathbb{R}^{n} \times\{0\} .
$$

In this paper, without further clarification, all embeddings of topological manifolds are assumed to be locally flat. In fact we will always assume (or prove) the existence of a normal microbundle which implies local flatness.

7.1.1. Microbundles. The discussion of topological transversality needs the concept of microbundles, which was introduced by Milnor [Mil64].

Definition 7.2. (Microbundles) Let $B$ be a topological space.

(1) A microbundle over a $B$ is a triple $(E, i, p)$ where $E$ is a topological space, $i: M \rightarrow E$ (the zero section map) and $p: E \rightarrow M$ (the projection) are continuous maps, satisfying the following conditions.

(a) $p \circ i=\operatorname{Id}_{M}$.

(b) For each $b \in B$ there exist an open neighborhood $U \subset B$ of $b$ and an open neighborhood $V \subset E$ of $i(b)$ with $i(U) \subset V, j(V) \subset U$, such that there is a homeomorphism $V \simeq U \times \mathbb{R}^{n}$ which makes the following diagram commutes.

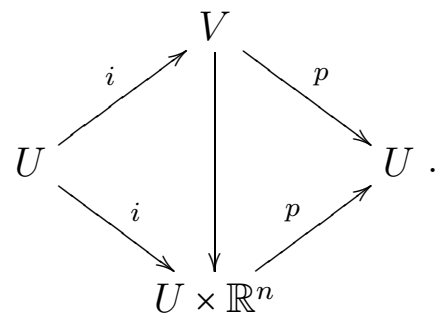

(2) Two microbundles $\xi=(E, i, p)$ and $\xi^{\prime}=\left(E^{\prime}, i^{\prime}, p^{\prime}\right)$ over $B$ are equivalent if there are open neighborhoods of the zero sections $W \subset E, W^{\prime} \subset E^{\prime}$ and a homeomorphism $\rho: W \rightarrow W^{\prime}$ which is compatible with the structures of the two microbundles.

Vector bundles and disk bundles are particular examples of microbundles. More generally, an $\mathbb{R}^{n}$-bundle over a topological manifold $M$ is a fibre bundle over $M$ whose fibres are $\mathbb{R}^{n}$ and whose structure group is the group of homeomorphisms of $\mathbb{R}^{n}$ which fix the origin. Notice that an $\mathbb{R}^{n}$-bundle has a continuous zero section, thus an $\mathbb{R}^{n}$-bundle is naturally a microbundle. A very useful fact, which was proved by Kister [Kis64] and Mazur [Maz64], says that microbundles are essentially $\mathbb{R}^{n}$-bundles. 
Theorem 7.3. (Kister-Mazur Theorem) Let B be a topological manifold (or a weaker space such as a locally finite simplicial complex) and $\xi=(E, i, p)$ be a microbundle over $B$. Then $\xi$ is equivalent to an $\mathbb{R}^{n}$-bundle, and the isomorphism class of this $\mathbb{R}^{n}$-bundle is uniquely determined by $\xi$.

However, $\mathbb{R}^{n}$-bundles are essentially different from vector bundles. For example, vector bundles always contain disk bundles, which is not true for $\mathbb{R}^{n}$-bundles.

Definition 7.4. (Normal microbundles) Let $f: S \rightarrow M$ be a topological embedding.

(1) A normal microbundle of $f$ is a pair $\xi=(N, \nu)$ where $N \subset M$ is an open neighborhood of $f(S)$ and $\nu: N \rightarrow S$ is a continuous map such that together with the natural inclusion $S \hookrightarrow N$ they form a microbundle over $N$. A normal microbundle is also called a tubular neighborhood.

(2) Two normal microbundles $\xi_{1}=\left(N_{1}, \nu_{1}\right)$ and $\xi_{2}=\left(N_{2}, \nu_{2}\right)$ are equivalent if there is another normal microbundle $(N, \nu)$ with $N \subset N_{1} \cap N_{2}$ and

$$
\left.\nu_{1}\right|_{N}=\left.\nu_{2}\right|_{N}=\nu .
$$

An equivalence class is called a germ of normal microbundles (or tubular neighborhoods).

For example, for a smooth submanifold $S \subset M$ in a smooth manifold, there is always a normal microbundle. Its equivalence class is not unique though, as we need to choose the projection map.

7.1.2. Transversality. We first recall the notion of microbundle transversality. Let $Y$ be a topological manifold, $X \subset Y$ be a submanifold and $\xi=(N, \nu)$ be a normal microbundle of $X$. Let $f: M \rightarrow Y$ be a continuous map.

Definition 7.5. (Microbundle transversality) Let $Y$ be a topological manifold, $X \subset Y$ be a submanifold and $\xi_{X}$ be a normal microbundle of $X$. Let $f: M \rightarrow Y$ be a continuous map. We say that $f$ is transverse to $\xi$ if the following conditions are satisfied.

(1) $f^{-1}(X)$ is a submanifold of $M$.

(2) There is a normal microbundle $\xi^{\prime}=\left(N^{\prime}, \nu^{\prime}\right)$ of $f^{-1}(X) \subset M$ such that the following diagram commute

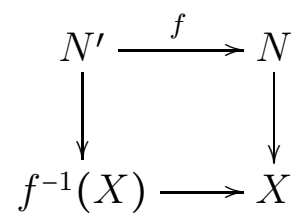

and the inclusion $f: N^{\prime} \rightarrow N$ induces an equivalence of microbundles.

More generally, if $C \subset M$ is any subset, then we say that $f$ is transverse to $X$ near $C$ if the restriction of $f$ to an open neighborhood of $C$ is transverse to $X$. $\xi$.

It is easy to see that the notion of being transverse to $\xi$ only depends on the germ of

Remark 7.6. The notion of microbundle transversality looks too restrictive at the first glance. For example, the line $x=y$ in $\mathbb{R}^{2}$ intersects transversely with the $x$-axis in the 
smooth category, however, the line is not transverse to the $x$-axis with respect to the natural normal microbundle given by the projection $(x, y) \rightarrow(x, 0)$.

The following theorem, which is of significant importance in our virtual cycle construction, shows that one can achieve transversality by arbitrary small perturbations.

Theorem 7.7. (Topological transversality theorem) Let $Y$ be a topological manifold and $X \subset Y$ be a proper submanifold. Let $\xi$ be a normal microbundle of $X$. Let $C \subset D \subset Y$ be closed sets. Suppose $f: M \rightarrow Y$ is a continuous map which is microbundle transverse to $\xi$ near $C$. Then there exists a homotopic map $g: M \rightarrow Y$ which is transverse to $\xi$ over $D$ such that the homotopy between $f$ and $g$ is supported in an open neighborhood of $f^{-1}((D \backslash C) \cap X)$.

Remark 7.8. The theorem was proved by Kirby-Siebenmann [KS77] with a restriction on the dimensions of $M, X$ and $Y$. Then Quinn [Qui82] [Qui88] [FQ90] completed the proof of the remaining cases. Notice that in [Qui88], the transversality theorem is stated for an embedding $i: M \rightarrow Y$ and the perturbation can be made through an isotopy. This implies the above transversality result for maps as we can identify a map $f: M \rightarrow Y$ with its graph $\tilde{f}: M \rightarrow M \times Y$, and an isotopic embedding of $\tilde{f}$, written as $\tilde{g}(x)=\left(g_{1}(x), g_{2}(x)\right)$, can be made transverse to the submanifold $\tilde{X}=M \times X \subset M \times Y$ with respect to the induced normal microbundle $\tilde{\xi}$. Then it is easy to see that it is equivalent to $g_{2}: M \rightarrow Y$ being transverse to $\xi$.

In most of the situations of this paper, the notion of transversality is about sections of vector bundles. Suppose $f: M \rightarrow \mathbb{R}^{n}$ is a continuous map. The origin $0 \in \mathbb{R}^{n}$ has a canonical normal microbundle. Therefore, one can define the notion of transversality for $f$ as a special case of Definition 7.5. Now suppose $E \rightarrow M$ is an $\mathbb{R}^{n}$-bundle and

$$
\varphi_{U}:\left.E\right|_{U} \rightarrow U \times \mathbb{R}^{n}
$$

is a local trivialization. Each section $s: M \rightarrow E$ induces a map $s_{U}: U \rightarrow \mathbb{R}^{n}$. Then we say that $s$ is transverse over $U$ if $s_{U}$ is transverse to the origin of $\mathbb{R}^{n}$. This notion is clearly independent of the choice of local trivializations. Then $s$ is said to be transverse if it is transverse over a sufficiently small neighborhood of every point of $M$.

Notice that the zero section of $E$ has a canonical normal microbundle in the total space, and this notion of transversality for sections never agrees with the notion of transversality for graphs of the sections with respect to this canonical normal microbundle. Hence there is an issue about whether this transversality notion for sections behaves as well as the microbundle transversality.

Theorem 7.9. Let $M$ be a topological manifold and $E \rightarrow M$ be an $\mathbb{R}^{n}$-bundle. Let $C \subset D \subset M$ be closed subsets. Let $s: M \rightarrow E$ be a continuous section which is transverse near $C$. Then there exists another continuous section which is transverse near $D$ and which agrees with s over a small neighborhood of $C$.

Proof. The difficulty is that the graph of $s$ is not microbundle transverse to the zero section, hence we cannot directly apply the topological transversality theorem (Theorem 7.7). Hence we need to use local trivializations view the section locally as a map into $\mathbb{R}^{n}$. For each $p \in D$, choose a precompact open neighborhood $U_{p} \subset M$ of $p$ and a local 
trivialization

$$
\varphi_{p}:\left.E\right|_{U_{p}} \simeq U_{p} \times \mathbb{R}^{n}
$$

All $U_{p}$ form an open cover of $D$. $M$ is paracompact, so is $D$. Hence there exists a locally finite refinement with induced local trivializations. Moreover, $D$ is Lindelöf, hence this refinement has a countable subcover, denoted by $\left\{U_{i}\right\}_{i=1}^{\infty}$. Over each $U_{i}$ there is an induced trivialization of $E$.

We claim that there exists precompact open subsets $V_{i} \sqsubset U_{i}$ such that $\left\{V_{i}\right\}_{i=1}^{\infty}$ still cover $D$. We construct $V_{i}$ inductively. Indeed, a topological manifold satisfies the $T_{4}$-axiom, hence we can use open sets to separate the two closed subsets

$$
D \backslash \bigsqcup_{i \neq 1} U_{i}, \quad D \backslash U_{1} \text {. }
$$

This provides a precompact $V_{i} \sqsubset U_{i}$ such that replacing $U_{1}$ by $V_{1}$ one still has an open cover of $D$. Suppose we can find $V_{1}, \ldots, V_{k}$ so that replacing $U_{1}, \ldots, U_{k}$ by $V_{1}, \ldots, V_{k}$ still gives an open cover of $D$. Then one can obtain $V_{k+1} \sqsubset U_{k+1}$ to continue the induction. We see that $\left\{V_{i}\right\}_{i=1}^{\infty}$ is an open cover of $D$ because every point $p \in D$ is contained in at most finitely many $U_{i}$.

Now take an open neighborhood $U_{C} \subset M$ of $C$ over which $s$ is transverse. Since $M$ is a manifold, one can separate the two closed subsets $C$ and $M \backslash U_{C}$ by a cut-off function

$$
\rho_{C}: M \rightarrow[-1,1]
$$

such that

$$
\rho_{C}^{-1}(-1)=C, \rho_{C}^{-1}(1)=M \backslash U_{C} .
$$

Define a sequence of open sets

$$
C^{k}=\rho_{C}^{-1}\left(\left[-1, \frac{1}{k+1}\right)\right) .
$$

Similarly, we can choose a sequence of shrinkings

$$
V_{i} \sqsubset \cdots \sqsubset V_{i}^{k+1} \sqsubset V_{i}^{k} \sqsubset \cdots \sqsubset U_{i} .
$$

Define

$$
W^{k}=C^{k} \cup \bigcup_{i=1}^{k} V_{i}^{k}
$$

which is a sequence of open subsets of $M$.

Now we start an inductive construction. First, over $U_{1}$, the section can be identified with a map $s_{1}: U_{1} \rightarrow \mathbb{R}^{n}$. By our assumption, $s_{1}$ is transverse over $U_{C} \cap U_{1}$. Then apply the theorem for the pair of closed subsets $\overline{C^{1}} \cap U_{1} \subset\left(\overline{C^{1}} \cap U_{1}\right) \cup \overline{V_{1}^{1}}$ of $U_{1}$. Then one can modify it so that it becomes transverse near $\left(\overline{C^{1}} \cap U_{1}\right) \cup \overline{V_{1}^{1}}$, and the change is only supported in a small neighborhood of $\overline{V_{1}^{1}} \backslash \overline{C^{1}}$. This modified section still agrees with the original section near the boundary of $U_{1}$, hence still defines a section of $E$. It also agrees with the original section over a neighborhood of $\overline{C^{2}}$. Moreover, the modified section is transverse near

$$
\overline{W^{1}}:=\overline{C^{1}} \cup \overline{V_{1}^{1}} \text {. }
$$

Now suppose we have modified the section so that it is transverse near

$$
\overline{W^{k}}:=\overline{C^{k}} \cup \bigcup_{i=1}^{k} \overline{V_{i}^{k}}
$$


and such that $s$ agrees with the original section over an open neighborhood of $\overline{C^{k+1}}$. Then by similar method, one can modify $s$ (via the local trivialization over $U_{k+1}$ ) to a section which agrees with $s$ over a neighborhood of

$$
\overline{C^{k+1}} \cup \bigcup_{i=1}^{k} \overline{V_{i}^{k+1}}
$$

and is transverse near $\overline{W^{k+1}}$. In particular this section still agrees with the very original section over $C^{k+1}$.

We claim that this induction process provides a section $s$ of $E$ which satisfies the requirement. Indeed, since the open cover $\left\{U_{i}\right\}_{i=1}^{\infty}$ of $D$ is locally finite, the value of the section becomes stabilized after finitely many steps of the induction, hence defines a continuous section. Moreover, in each step the value of the section remains unchanged over the open set $\rho_{C}^{-1}([-1,0))$. Transversality also holds by construction.

Corollary 7.10. Let $M$ be a topological manifold with or without boundary, and let $s_{1}, s_{2}: M \rightarrow E$ be two transverse sections which are homotopic. Then the two submanifolds (with or without boundary) $S_{1}:=s_{1}^{-1}(0)$ and $S_{2}:=s_{2}^{-1}(0)$ are cobordant.

Remark 7.11. In the application of this paper, the target pair $(X, Y)$ in the transversality problem is either a smooth submanifold inside a smooth manifold (or orbifolds), or the zero section of a vector bundle. Hence $X$ admits a tubular neighborhood and a unique equivalence class of normal microbundle. In fact the normal microbundle is equivalent to a disk bundle of the smooth normal bundle. Hence in the remaining discussions, we make the stronger assumption that all normal microbundles are disk bundles of some vector bundle. This does not alter the above discussion. For example, the compositions of two embeddings with disk bundle neighborhoods is still an embedding with a disk bundle neighborhood.

7.2. Topological orbifolds and orbibundles. We use Satake's notion of V-manifolds [Sat56] instead of groupoids to treat orbifolds, and only discuss it in the topological category. In this paper we only consider effective orbifolds.

Definition 7.12. Let $M$ be a second countable Hausdorff topological space.

(1) Let $x \in M$ be a point. A topological orbifold chart (with boundary) of $x$ consists of a triple $\left(\tilde{U}_{x}, \Gamma_{x}, \varphi_{x}\right)$, where $\tilde{U}_{x}$ is a topological manifold with possibly empty boundary $\partial \tilde{U}_{x}, \Gamma_{x}$ is a finite group acting continuously on $\left(\tilde{U}_{x}, \partial \tilde{U}_{x}\right)$ and

$$
\varphi_{x}: \tilde{U}_{x} / \Gamma_{x} \rightarrow M
$$

is a continuous map which is a homeomorphism onto an open neighborhood of $x$. Denote the image $U_{x}=\varphi_{x}\left(\tilde{U}_{x} / \Gamma_{x}\right) \subset M$ and denote the composition

$$
\tilde{\varphi}_{x}: \tilde{U}_{x} \longrightarrow \tilde{U}_{x} / \Gamma_{x} \stackrel{\varphi_{x}}{\longrightarrow} M .
$$

(2) If $p \in \tilde{U}_{x}$, take $\Gamma_{p}=\left(\Gamma_{x}\right)_{p} \subset \Gamma_{x}$ the stabilizer of $p$. Let $\tilde{U}_{p} \subset \tilde{U}_{x}$ be a $\Gamma_{p}$-invariant neighborhood of $p$. Then there is an induced chart (which we call a subchart) $\left(\tilde{U}_{p}, \Gamma_{p}, \varphi_{p}\right)$, where $\varphi_{p}$ is the composition

$$
\varphi_{p}: \tilde{U}_{p} / \Gamma_{p} \longleftrightarrow \tilde{U}_{x} / \Gamma_{x} \stackrel{\varphi_{p}}{\longrightarrow} M .
$$


(3) Two charts $\left(\tilde{U}_{x}, \Gamma_{x}, \varphi_{x}\right)$ and $\left(\tilde{U}_{y}, \Gamma_{y}, \varphi_{y}\right)$ are compatible if for any $p \in \tilde{U}_{x}$ and $q \in \tilde{U}_{y}$ with $\varphi_{x}(p)=\varphi_{y}(q) \in M$, there exist an isomorphism $\Gamma_{p} \rightarrow \Gamma_{q}$, subcharts $\tilde{U}_{p} \ni p, \tilde{U}_{q} \ni q$ and an equivariant homeomorphism $\varphi_{q p}:\left(\tilde{U}_{p}, \partial \tilde{U}_{p}\right) \simeq\left(\tilde{U}_{q}, \partial \tilde{U}_{q}\right)$.

(4) A topological orbifold atlas of $M$ is a set $\left\{\left(\tilde{U}_{\alpha}, \Gamma_{\alpha}, \varphi_{\alpha}\right) \mid \alpha \in I\right\}$ of topological orbifold charts of $M$ such that $M=\bigcup_{\alpha \in I} U_{\alpha}$ and for each pair $\alpha, \beta \in I$, $\left(\tilde{U}_{\alpha}, \Gamma_{\alpha}, \varphi_{\alpha}\right),\left(\tilde{U}_{\beta}, \Gamma_{\beta}, \varphi_{\beta}\right)$ are compatible. Two atlases are equivalent if the union of them is still an atlas. A structure of topological orbifold (with boundary) is an equivalence class of atlases. A topological orbifold (with boundary) is a second countable Hausdorff space with a structure of topological orbifold (with boundary).

We will often skip the term "topological" in the rest of this paper.

Now consider bundles. Let $E, B$ be orbifolds and $\pi: E \rightarrow B$ be a continuous map.

Definition 7.13. A vector bundle chart (resp. disk bundle chart) of $\pi: E \rightarrow B$ is a tuple $\left(\tilde{U}, F^{n}, \Gamma, \hat{\varphi}, \varphi\right)$ where $F^{n}=\mathbb{R}^{n}$ (resp. $\left.F^{n}=\mathbb{D}^{n}\right),(\tilde{U}, \Gamma, \varphi)$ is a chart of $B$ and $\left(\tilde{U} \times F^{n}, \Gamma, \hat{\varphi}\right)$ is a chart of $E$, where $\Gamma$ acts on $F^{n}$ via a representation $\Gamma \rightarrow G L\left(\mathbb{R}^{n}\right)$ (resp. $\Gamma \rightarrow O(n))$. The compatibility condition is required, namely, the following diagram commutes.

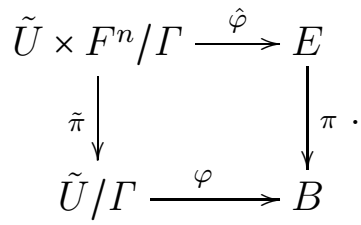

If $\left(\tilde{U}_{p}, \Gamma_{p}, \varphi_{p}\right)$ is a subchart of $(\tilde{U}, \Gamma, \varphi)$, then one can restrict the bundle chart to $\tilde{\pi}^{-1}\left(\tilde{U}_{p}\right)$.

We can define the notion of compatibility between bundle charts, the notion of orbifold bundle structures and the notion of orbifold bundles in a similar fashion as in the case of orbifolds. We skip the details.

7.2.1. Embeddings. Now we consider embeddings for orbifolds and orbifold vector bundles. First we consider the case of manifolds. Let $S$ and $M$ be topological manifolds and $E \rightarrow S, F \rightarrow M$ be continuous vector bundles. Let $\phi: S \rightarrow M$ be a topological embedding. A bundle embedding covering $\phi$ is a continuous map $\widehat{\phi}: E \rightarrow F$ which makes the diagram

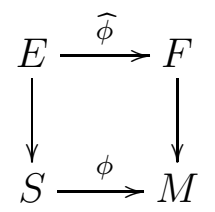

commute and which is fibrewise a linear injective map. Since $\widehat{\phi}$ determines $\phi$, we also call $\widehat{\phi}: E \rightarrow F$ a bundle embedding.

Definition 7.14. (Orbifold embedding) Let $S, M$ be orbifolds and $f: S \rightarrow M$ is a continuous map which is a homeomorphism onto its image. $\phi$ is called an embedding if for any pair of orbifold charts, $(\tilde{U}, \Gamma, \varphi)$ of $S$ and $(\tilde{V}, \Pi, \psi)$ of $M$, any pair of points 
$p \in \tilde{U}, q \in \tilde{V}$ with $\phi(\varphi(p))=\psi(q)$, there are subcharts $\left(\tilde{U}_{p}, \Gamma_{p}, \varphi_{p}\right) \subset(\tilde{U}, \Gamma, \varphi)$ and $\left(\tilde{V}_{q}, \Pi_{q}, \psi_{q}\right) \subset(\tilde{V}, \Pi, \psi)$, an isomorphism $\Gamma_{p} \simeq \Pi_{q}$ and an equivariant locally flat embed$\operatorname{ding} \tilde{\phi}_{p q}: \tilde{U}_{p} \rightarrow \tilde{V}_{q}$ such that the following diagram commutes.

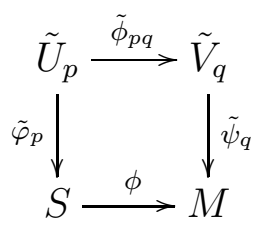

7.2.2. Multisections and perturbations. The equivariant feature of the problem implies that transversality can only be achieved by multi-valued perturbations. Here we review basic notions and facts about multisections. Our discussion mainly follows the treatment of [FO99].

Definition 7.15. (Multimaps) Let $A, B$ be sets, $l \in \mathbb{N}$, and $\mathcal{S}^{l}(B)$ be the $l$-fold symmetric product of $B$.

(1) An l-multimap $f$ from $A$ to $B$ is a map $f: A \rightarrow \mathcal{S}^{l}(B)$. For another $a \in \mathbb{N}$, there is a natural map

$$
m_{a}: \mathcal{S}^{l}(B) \rightarrow \mathcal{S}^{a l}(B)
$$

by repeating each component $a$ times.

(2) If both $A$ and $B$ are acted by a finite group $\Gamma$, then we say that an $l$-multimap $f: A \rightarrow \mathcal{S}^{l}(B)$ is $\Gamma$-equivariant if it is equivariant with respect to the $\Gamma$-action on $A$ and the induced $\Gamma$-action on $\mathcal{S}^{l}(B)$.

(3) If $A$ and $B$ are both topological spaces, then an $l$-multimap $f: A \rightarrow \mathcal{S}^{l}(B)$ is called continuous if it is continuous with respect to the topology on $\mathcal{S}^{l}(B)$ induced as a quotient of $B^{l}$.

(4) A continuous l-multimap $f: A \rightarrow \mathcal{S}^{l}(B)$ is liftable if there are continuous maps $f_{1}, \ldots, f_{l}: A \rightarrow B$ such that

$$
f(x)=\left[f^{1}(x), \ldots, f^{l}(x)\right] \in \mathcal{S}^{l}(B), \forall x \in A .
$$

$f^{1}, \ldots, f^{l}$ are called branches of $f$.

(5) An $l_{1}$-multimap $f_{1}: A \rightarrow \mathcal{S}^{l_{1}}(B)$ and an $l_{2}$-multimap $f_{2}: A \rightarrow \mathcal{S}^{l_{2}}(B)$ are called equivalent if there exists a common multiple $l=a_{1} l_{1}=a_{2} l_{2}$ of $l_{1}$ and $l_{2}$ such that

$$
m_{a_{1}} \circ f_{1}=m_{a_{2}} \circ f_{2}
$$

as $l$-multimaps from $A$ to $B$.

(6) Being equivalent is clearly reflexive, symmetric and transitive. A multimap from $A$ to $B$, denoted by $f: A \stackrel{m}{\rightarrow} B$, is an element of

$$
\left(\bigsqcup_{l \geq 1} \operatorname{Map}\left(A, \mathcal{S}^{l}(B)\right)\right) / \sim
$$

where $\sim$ is the above equivalence relation.

In the discussions in this paper, we often identify an $l$-multimap with its equivalence class as a multimap.

Definition 7.16. (Multisections) Let $M$ be a topological orbifold and $E \rightarrow M$ be a vector bundle. 
(1) A representative of a (continuous) multisection of $E$ is a collection

$$
\left\{\left(\tilde{U}_{\alpha}, \mathbb{R}^{k}, \Gamma_{\alpha}, \hat{\varphi}_{\alpha}, \varphi_{\alpha} ; s_{\alpha}, l_{\alpha}\right) \mid \alpha \in I\right\}
$$

where $\left\{\left(\tilde{U}_{\alpha}, \mathbb{R}^{k}, \Gamma_{\alpha}, \hat{\varphi}_{\alpha}, \varphi_{\alpha}\right) \mid \alpha \in I\right\}$ is a bundle atlas for $E \rightarrow M$ and $s_{\alpha}: \tilde{U}_{\alpha} \rightarrow$ $\mathcal{S}^{l_{\alpha}}\left(\mathbb{R}^{k}\right)$ is a $\Gamma_{\alpha}$-equivariant continuous $l_{\alpha}$-multimap, satisfying the following compatibility condition.

- For any $p \in \tilde{U}_{\alpha}$ and $q \in \tilde{U}_{\beta}$ with $\varphi_{\alpha}(p)=\varphi_{\beta}(q) \in M$, there exist subcharts $\tilde{U}_{p} \subset \tilde{U}_{\alpha}, \tilde{U}_{q} \subset \tilde{U}_{\beta}$, an isomorphism $\left(\hat{\varphi}_{p q}, \varphi_{p q}\right)$ of subcharts, a common multiple $l=a_{\alpha} l_{\alpha}=a_{\beta} l_{\beta}$ of $l_{\alpha}$ and $l_{\beta}$, such that

$$
\left.\hat{\varphi}_{p q} \circ m_{a_{\beta}} \circ s_{\beta}\right|_{\tilde{U}_{q}}=\left.m_{a_{\alpha}} \circ s_{\alpha}\right|_{\tilde{U}_{p}} \circ \varphi_{p q} .
$$

(2) Two representatives are equivalent if their union is also a representative. An equivalence class is called a multisection of $E$, denoted by

$$
s: O \stackrel{m}{\rightarrow} E .
$$

(3) A multisection $s: M \stackrel{m}{\rightarrow} E$ is called locally liftable if for any $p \in M$, there exists a local representative $\left(\tilde{U}_{p} \times \mathbb{R}^{k}, \Gamma_{p}, \hat{\varphi}_{p}, \varphi_{p} ; s_{p}, l_{p}\right)$ such that $s_{p}: \tilde{U}_{p} \rightarrow \mathcal{S}^{l_{p}}\left(\mathbb{R}^{k}\right)$ which is a liftable continuous $l_{p}$-multimap.

(4) A multisection $s: M \stackrel{m}{\rightarrow} E$ is called transverse if it is locally liftable and for any liftable local representative $s_{p}: \tilde{U}_{p} \rightarrow \mathcal{S}^{l}\left(\mathbb{R}^{k}\right)$, all branches are transverse to the origin of $\mathbb{R}^{k}$.

The space of continuous multisections of $E \rightarrow M$, denoted by $C_{m}^{0}(M, E)$, is acted by the space of continuous functions $C^{0}(M)$ on $M$ by pointwise multiplication. $C_{m}^{0}(M, E)$ also has the structure of a commutative monoid, but not an abelian group. The additive structure is defined as follows. If $s_{1}, s_{2}: M \stackrel{m}{\rightarrow} E$ are multisections, then for liftable local representatives with branches $s_{1}^{a}: \tilde{U} \rightarrow \mathbb{R}^{n}, 1 \leq a \leq l, s_{2}^{b}: \tilde{U} \rightarrow \mathbb{R}^{n}, 1 \leq b \leq k$, define

$$
\left(s_{1}+s_{2}\right)^{a b}=\left[s_{1}^{a}+s_{2}^{b}\right]_{1 \leq a \leq l}^{1 \leq b \leq k} .
$$

However there is no inverse to this addition: one can only invert the operation of adding a single valued section. It is enough, though, since we have the notion of being transverse to a single valued section which is not necessarily the zero section.

We also want to measure the size of multisections. A continuous norm on an orbifold vector bundle $E \rightarrow M$ is a continuous function $\|\cdot\|: E \rightarrow[0,+\infty)$ which only vanishes on the zero section such that over each local chart, it lifts to an equivariant norm on the fibres. It is easy to construct norms in the relative sense, as one can extend continuous functions defined on closed sets.

The following lemma, which is a generalization of Theorem 7.9, shows one can achieve transversality for multisections by perturbation relative to a region where transversality already holds.

Lemma 7.17. Let $M$ be an orbifold and $E \rightarrow M$ be an orbifold vector bundle. Let $C \subset D \subset M$ be closed subsets. Let $S: M \rightarrow E$ be a single-valued continuous function and $t_{C}: M \stackrel{m}{\rightarrow} E$ be a multisection such that $S+t_{C}$ is transverse over a neighborhood of $C$. Then there exists a multisection $t_{D}: M \stackrel{m}{\rightarrow} E$ satisfying the following condition.

(1) $t_{C}=t_{D}$ over a neighborhood of $C$. 
(2) $S+t_{D}$ is transverse over a neighborhood of $D$.

Moreover, if $E$ has a continuous norm $\|\cdot\|$, then for any $\epsilon>0$, one can require that

$$
\left\|t_{D}\right\|_{C^{0}} \leq\left\|t_{C}\right\|_{C^{0}}+\epsilon .
$$

Proof. Similar to the proof of Theorem 7.9, one can choose a countable locally finite open cover $\left\{U_{i}\right\}_{i=1}^{\infty}$ of $D$ satisfying the following conditions.

(1) Each $\overline{U_{i}}$ is compact.

(2) There are a collection of precompact open subsets $V_{i} \sqsubset U_{i}$ such that $\left\{V_{i}\right\}_{i=1}^{\infty}$ is still an open cover of $D$.

(3) Over each $U_{i}$ there is a local representative of $t_{C}$, written as

$$
\left(\tilde{U}_{i} \times \mathbb{R}^{k}, \Gamma_{i}, \hat{\varphi}_{i}, \varphi_{i} ; t_{i}, l_{i}\right)
$$

where $t_{i}: \tilde{U}_{i} \rightarrow \mathcal{S}^{l_{i}}\left(\mathbb{R}^{k}\right)$ is a $\Gamma_{i}$-equivariant $l_{i}$-multimap which is liftable. Write

$$
t_{i}(x)=\left[t_{i}^{1}(x), \ldots, t_{i}^{l_{i}}(x)\right] .
$$

In this chart also write $S$ as a map $S_{i}: \tilde{U}_{i} \rightarrow \mathbb{R}^{k}$.

The transversality assumption implies that there is an open neighborhood $U_{C} \subset M$ of $C$ such that for each $i$, each $a \in\left\{1, \ldots, l_{i}\right\}, s_{i}^{a}$ is transverse to the origin over

$$
\tilde{U}_{i, C}:=\varphi_{i}^{-1}\left(U_{C}\right) \subset \tilde{U}_{C}
$$

Using an inductive construction which is very similar to that in the proof of Theorem 7.9, one can construct a valid perturbation. We only sketch the construction for the first chart. Indeed, one can perturb each $t_{1}^{a}$ over $\tilde{U}_{1}$ to a function $\dot{t}_{1}^{a}: \tilde{U}_{1} \rightarrow \mathbb{R}^{k}$, such that $S_{1}+\dot{t}_{1}^{a}$ is transverse over a neighborhood of the closure of $\tilde{V}_{1}:=\varphi_{1}^{-1}\left(V_{1}\right)$ inside $\tilde{U}_{1}$, but $\dot{t}_{1}^{a}=t_{1}^{a}$ over a neighborhood of $\tilde{U}_{1, C}$ and the near the boundary of $\tilde{U}_{1}$. Moreover, given $\epsilon>0$ we may require that

$$
\sup _{x \in \tilde{U}_{1}}\left\|\dot{t}_{1}^{a}(x)\right\| \leq \sup _{x \in \tilde{U}_{1}}\left\|t_{1}^{a}(x)\right\|+\frac{\epsilon}{2} .
$$

Then we obtain a continuous $l_{1}$-multimap

$$
\dot{t}_{1}(x)=\left[\dot{t}_{1}^{1}(x), \ldots, \dot{t}_{1}^{l_{1}}(x)\right] .
$$

This multimap may not be $\Gamma_{1}$-transverse. We reset

$$
\dot{t}_{1}(x):=\left[t_{1}^{a b}\right]_{1 \leq a \leq l_{1}}^{1 \leq b \leq n_{1}}:=\left[g_{b}^{-1} \dot{t}_{1}^{a}\left(g_{b} x\right)\right]_{1 \leq a \leq l_{1}}^{1 \leq b \leq n_{1}}
$$

where $\Gamma_{1}=\left\{g_{1}, \ldots, g_{n_{1}}\right\}$. It is easy to verify that this is $\Gamma_{1}$-invariant, and agrees with $t_{1}$ over a neighborhood of $\tilde{U}_{1, C}$ and near the boundary of $\tilde{U}_{1}$. There still holds

$$
\sup _{a, b} \sup _{x \in \tilde{U}_{1}}\left\|\dot{t}_{1}^{a b}(x)\right\| \leq \sup _{a} \sup _{x \in \tilde{U}_{1}}\left\|t_{1}^{a}(x)\right\|+\frac{\epsilon}{2} .
$$

Therefore, together with the original multisection over the complement of $U_{1}, \dot{t}_{1}$ defines a continuous multisection of $E$. Moreover, it agrees with the original one over a neighborhood of $C$ and is transverse near $C \cup \overline{V_{1}}$. In this way we can continue the induction to perturb over all $\tilde{U}_{i}$. At the $k$-th step of the induction, we replace $\frac{\epsilon}{2}$ by $\frac{\epsilon}{2^{k}}$ in the $C^{0}$ bound (7.2). Since $U_{i}$ is locally finite, near each point, the value of the perturbation 
stabilizes after finitely many steps of this induction. This results in a multisection $t_{D}$ which satisfies our requirement.

7.3. Virtual orbifold atlases. Now we introduce the notion of virtual orbifold atlases. This notion plays a role as a general structure of moduli spaces we are interested in, and is a type of intermediate objects in concrete constructions. The eventual objects we would like to construct are good coordinate systems, which are special types of virtual orbifold atlases.

Definition 7.18. Let $X$ be a compact and Hausdorff space.

(1) A virtual orbifold chart (chart for short) is a tuple

$$
C:=(U, E, S, \psi, F)
$$

where

(a) $U$ is a topological orbifold (with boundary).

(b) $E \rightarrow U$ is a continuous orbifold vector bundle.

(c) $S: U \rightarrow E$ is a continuous section.

(d) $F \subset X$ is an open subset.

(e) $\psi: S^{-1}(0) \rightarrow F$ is a homeomorphism.

$F$ is call the footprint of this chart $C$, and the integer $\operatorname{dim} U-\operatorname{rank} E$ is called the virtual dimension of $C$.

(2) Let $C=(U, E, S, \psi, F)$ be a chart and $U^{\prime} \subset U$ be an open subset. The restriction of $C$ to $U^{\prime}$ is the chart

$$
C^{\prime}=\left.C\right|_{U^{\prime}}=\left(U^{\prime}, E^{\prime}, S^{\prime}, \psi^{\prime}, F^{\prime}\right)
$$

where $E^{\prime}=\left.E\right|_{U^{\prime}}, S^{\prime}=\left.S\right|_{U^{\prime}}, \psi^{\prime}=\left.\psi\right|_{\left(S^{\prime}\right)^{-1}(0)}$, and $F^{\prime}=$ Image $\psi^{\prime}$. Any such chart $C^{\prime}$ induced from an open subset $U^{\prime} \subset U$ is called a shrinking of $C$. A shrinking $C^{\prime}=\left.C\right|_{U^{\prime}}$ is called a precompact shrinking if $U^{\prime} \sqsubset U$, denoted by $C^{\prime} \sqsubset C$.

A very useful lemma about shrinkings is the following, whose proof is left to the reader.

Lemma 7.19. Suppose $C=(U, E, S, \psi, F)$ is a virtual orbifold atlas and let $F^{\prime} \subset F$ be an open subset. Then there exists a shrinking $C^{\prime}$ of $C$ whose footprints is $F^{\prime}$. Moreover, if $F^{\prime} \sqsubset F$, then $C^{\prime}$ can be chosen to be a precompact shrinking.

Definition 7.20. Let $C_{i}:=\left(U_{i}, E_{i}, S_{i}, \psi_{i}, F_{i}\right), i=1,2$ be two charts of $X$. An embedding of $C_{1}$ into $C_{2}$ consists of a bundle embedding $\widehat{\phi}_{21}$ satisfying the following conditions.

(1) The following diagrams commute;
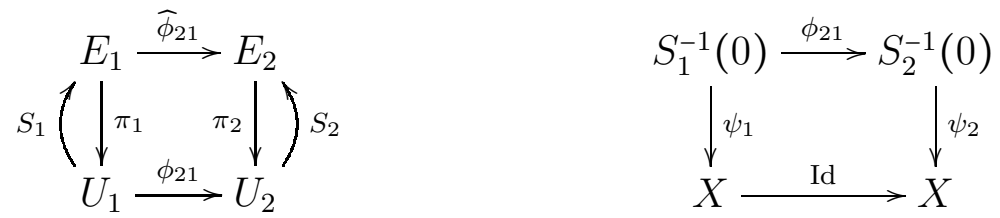

(2) (Tangent Bundle Condition) There exists an open neighborhood $N_{21} \subset U_{2}$ of $\phi_{21}\left(U_{1}\right)$ and a subbundle $\left.E_{1 ; 2} \subset E_{2}\right|_{N_{21}}$ which extends $\widehat{\phi}_{21}\left(E_{1}\right)$ such that $\left.S_{2}\right|_{N_{2}}:\left.N_{2} \rightarrow E_{2}\right|_{N_{2}}$ is transverse to $E_{1 ; 2}$ and $S_{2}^{-1}\left(E_{1 ; 2}\right)=\phi_{21}\left(U_{1}\right)$. 
The following lemma is left to the reader.

Lemma 7.21. The composition of two embeddings is still an embedding.

Definition 7.22. Let $C_{i}=\left(U_{i}, E_{i}, S_{i}, \psi_{i}, F_{i}\right),(i=1,2)$ be two charts. A coordinate change from $C_{1}$ to $C_{2}$ is a triple $T_{21}=\left(U_{21}, \phi_{21}, \widehat{\phi}_{21}\right)$, where $U_{21} \subset U_{1}$ is an open subset and $\left(\phi_{21}, \widehat{\phi}_{21}\right)$ is an embedding from $\left.C_{1}\right|_{U_{21}}$ to $C_{2}$. They should satisfy the following conditions.

(1) $\psi_{1}\left(U_{21} \cap S_{1}^{-1}(0)\right)=F_{1} \cap F_{2}$.

(2) If $x_{k} \in U_{21}$ converges to $x_{\infty} \in U_{1}$ and $y_{k}=\phi_{21}\left(x_{k}\right)$ converges to $y_{\infty} \in U_{2}$, then $x_{\infty} \in U_{21}$ and $y_{\infty}=\phi_{21}\left(y_{\infty}\right)$.

Lemma 7.23. Let $C_{i}=\left(U_{i}, E_{i}, S_{i}, \psi_{i}, F_{i}\right),(i=1,2)$ be two charts and let $T_{21}=\left(U_{21}, \widehat{\phi}_{21}\right)$ be a coordinate change from $C_{1}$ to $C_{2}$. Suppose $C_{i}^{\prime}=\left.C_{i}\right|_{U_{i}^{\prime}}$ be a shrinking of $C_{i}$. Then the restriction $T_{21}^{\prime}:=\left.T_{21}\right|_{U_{1}^{\prime} \cap \phi_{21}^{-1}\left(U_{2}^{\prime}\right)}$ is a coordinate change from $C_{1}^{\prime}$ to $C_{2}^{\prime}$.

Proof. Left to the reader.

We call $T_{21}^{\prime}$ in the above lemma the induced coordinate change from the shrinking.

Now we introduce the notion of atlases.

Definition 7.24. Let $X$ be a compact metrizable space. A virtual orbifold atlas of virtual dimension $d$ on $X$ is a collection

$$
\mathfrak{A}:=\left(\left\{C_{I}:=\left(U_{I}, E_{I}, S_{I}, \psi_{I}, F_{I}\right) \mid I \in \mathcal{I}\right\},\left\{T_{J I}=\left(U_{J I}, \phi_{J I}, \widehat{\phi}_{J I}\right) \mid I \leqslant J\right\}\right),
$$

where

(1) $(\mathcal{I}, \leqslant)$ is a finite, partially ordered set.

(2) For each $I \in \mathcal{I}, C_{I}$ is a virtual orbifold chart of virtual dimension $d$ on $X$.

(3) For $I \leqslant J, T_{J I}$ is a coordinate change from $C_{I}$ to $C_{J}$.

They are subject to the following conditions.

- (Covering Condition) $X$ is covered by all the footprints $F_{I}$.

- (Cocycle Condition) For $I \leqslant J \leqslant K \in \mathcal{I}$, denote $U_{K J I}=U_{K I} \cap \phi_{J I}^{-1}\left(U_{K J}\right) \subset U_{I}$. Then we require that

$$
\left.\widehat{\phi}_{K I}\right|_{U_{K J I}}=\left.\widehat{\phi}_{K J} \circ \widehat{\phi}_{J I}\right|_{U_{K J I}}
$$

as bundle embeddings.

- (Overlapping Condition) For $I, J \in \mathcal{I}$, we have

$$
\overline{F_{I}} \cap \overline{F_{J}} \neq \varnothing \Longrightarrow I \leqslant J \text { or } J \leqslant I \text {. }
$$

All virtual orbifold atlases considered in this paper have definite virtual dimensions, although sometimes we do not explicitly mention it.

7.3.1. Orientations. Now we discuss orientation. When $M$ is a topological manifold, there is an orientation bundle $\mathcal{O}_{M} \rightarrow M$ which is a double cover of $M$ (or a $\mathbb{Z}_{2}$-principal bundle). $M$ is orientable if and only if $\mathcal{O}_{M}$ is trivial. If $E \rightarrow M$ is a continuous vector bundle, then $E$ also has an orientation bundle $\mathcal{O}_{E} \rightarrow M$ as a double cover. Since $\mathbb{Z}_{2^{-}}$ principal bundles over a base $B$ are classified by $H^{1}\left(B ; \mathbb{Z}_{2}\right)$, the orientation bundles can be multiplied. We use $\otimes$ to denote this multiplication. 
Definition 7.25. (Orientability)

(1) A virtual orbifold chart $C=(U, E, S, \psi, F)$ is locally orientable if for any bundle chart $\left(\tilde{U}, \mathbb{R}^{n}, \Gamma, \widehat{\varphi}, \varphi\right)$ of $E$, if we denote $\tilde{E}=\tilde{U} \times \mathbb{R}^{n}$, then for any $\gamma \in \Gamma$, the map

$$
\gamma: \mathcal{O}_{\tilde{U}} \otimes \mathcal{O}_{\tilde{E}^{*}} \rightarrow \mathcal{O}_{\tilde{U}} \otimes \mathcal{O}_{\tilde{E}^{*}}
$$

is the identity over all fixed points of $\gamma$.

(2) If $C$ is locally orientable, then $\mathcal{O}_{\tilde{U}} \otimes \mathcal{O}_{\tilde{E}^{*}}$ for all local charts glue together a double cover $\mathcal{O}_{C} \rightarrow U$. If $\mathcal{O}_{C}$ is trivial (resp. trivialized), then we say that $C$ is orientable (resp. oriented).

(3) A coordinate change $T_{21}=\left(U_{21}, \phi_{21}, \widehat{\phi}_{21}\right)$ between two oriented charts $C_{1}=$ $\left(U_{1}, E_{1}, S_{1}, \psi_{1}, F_{1}\right)$ and $C_{2}=\left(U_{2}, E_{2}, S_{2}, \psi_{2}, F_{2}\right)$ is called oriented if the embeddings $\phi_{21}$ and $\widehat{\phi}_{21}$ are compatible with the orientations on $\mathcal{O}_{C_{1}}$ and $\mathcal{O}_{C_{2}}$.

(4) An atlas $\mathfrak{A}$ is oriented if all charts are oriented and all coordinate changes are oriented.

7.4. Good coordinate systems. Now we introduce the notion of shrinkings of virtual orbifold atlases.

Definition 7.26. Let $\mathfrak{A}=\left(\left\{C_{I} \mid I \in \mathcal{I}\right\},\left\{T_{J I} \mid I \leqslant J\right\}\right)$ be a virtual orbifold atlas on $X$.

(1) A shrinking of $\mathfrak{A}$ is another virtual orbifold atlas $\mathfrak{A}^{\prime}=\left(\left\{C_{I}^{\prime} \mid I \in \mathcal{I}\right\},\left\{T_{J I}^{\prime} \mid I \leqslant J\right\}\right)$ indexed by elements of the same partially ordered set $\mathcal{I}$ such that for each $I \in \mathcal{I}$, $C_{I}^{\prime}$ is a shrinking $\left.C_{I}\right|_{U_{I}^{\prime}}$ of $C_{I}$ and for each $I \leqslant J, T_{J I}^{\prime}$ is the induced shrinking of $T_{J I}$ given by Lemma 7.23 .

(2) If for every $I \in \mathcal{I}, U_{I}^{\prime}$ is a precompact subset of $U_{I}$, then we say that $\mathfrak{A}^{\prime}$ is a precompact shrinking of $\mathfrak{A}$ and denote $\mathfrak{A}^{\prime} \sqsubset \mathfrak{A}$.

Given a virtual orbifold atlas $\mathfrak{A}=\left(\left\{C_{I} \mid I \in \mathcal{I}\right\},\left\{T_{J I} \mid I \leqslant J\right\}\right)$, we define a relation $\vee$ on the disjoint union $\bigsqcup_{I \in \mathcal{I}} U_{I}$ as follows. $U_{I} \ni x \vee y \in U_{J}$ if one of the following holds.

(1) $I=J$ and $x=y$;

(2) $I \leqslant J, x \in U_{J I}$ and $y=\phi_{J I}(x)$;

(3) $J \leqslant I, y \in U_{I J}$ and $x=\phi_{I J}(y)$.

If $\mathfrak{A}^{\prime}$ is a shrinking of $\mathfrak{A}$, then it is easy to see that the relation $\vee^{\prime}$ on $\bigsqcup_{I \in \mathcal{I}} U_{I}^{\prime}$ defined as above is induced from the relation $\vee$ for $\mathfrak{A}$ via restriction.

For an atlas $\mathfrak{A}$, if $\vee$ is an equivalence relation, we can form the quotient space

$$
|\mathfrak{A}|:=\left(\bigsqcup_{I \in \mathcal{I}} U_{I}\right) / \vee .
$$

with the quotient topology. There is a natural injective map

$$
X \leftrightarrow|\mathfrak{A}| .
$$

We call $|\mathfrak{A}|$ the virtual neighborhood of $X$ associated to the atlas $\mathfrak{A}$. Denote the quotient map by

$$
\pi_{\mathfrak{A}}: \bigsqcup_{I \in \mathcal{I}} U_{I} \rightarrow|\mathfrak{A}|
$$

which induces continuous injections $U_{I} \hookrightarrow|\mathfrak{A}|$. A point in $|\mathfrak{A}|$ is denoted by $|x|$, which has certain representative $x \in U_{I}$ for some $I$. 
Definition 7.27. A virtual orbifold atlas $\mathfrak{A}$ on $X$ is called a good coordinate system if the following conditions are satisfied.

(1) $\vee$ is an equivalence relation.

(2) The virtual neighborhood $|\mathfrak{A}|$ is a Hausdorff space.

(3) For all $I \in \mathcal{I}$, the natural maps $U_{I} \rightarrow|\mathfrak{A}|$ are homeomorphisms onto their images.

The conditions for good coordinate systems are very useful for later constructions (this is the same as in the Kuranishi approach, see [FOOO16]), for example, the construction of suitable multisection perturbations. In these constructions, the above conditions are often implicitly used without explicit reference. Therefore, an important step is to construct good coordinate systems.

Theorem 7.28. (Constructing good coordinate system) Let $\mathfrak{A}$ be a virtual orbifold atlas on $X$ with the collection of footprints $F_{I}$ indexed by $I \in \mathcal{I}$. Let $F_{I}^{\square} \sqsubset F_{I}$ for all $I \in \mathcal{I}$ be a collection of precompact open subsets such that

$$
X=\bigcup_{I \in \mathcal{I}} F_{I}^{\square} \text {. }
$$

Then there exists a shrinking $\mathfrak{A}^{\prime}$ of $\mathfrak{A}$ such that the collection of shrunk footprints $F_{I}^{\prime}$ contains $\overline{F_{I}^{\square}}$ for all $I \in \mathcal{I}$ and $\mathfrak{A}^{\prime}$ is a good coordinate system.

Moreover, if $\mathfrak{A}$ is already a good coordinate system, then any shrinking of $\mathfrak{A}$ remains a good coordinate system.

We give a proof of Theorem 7.28 in the next subsection. A similar result is used in the Kuranishi approach while our argument potentially differs from that of [FOOO16].

Remark 7.29. If $\mathfrak{A}$ is a good coordinate system, and $\mathfrak{A}^{\prime}$ is a shrinking of $\mathfrak{A}$, then the shrinking induces a natural map

$$
\left|\mathfrak{A}^{\prime}\right| \hookrightarrow|\mathfrak{A}| \text {. }
$$

If we equip both $\left|\mathfrak{A}^{\prime}\right|$ and $|\mathfrak{A}|$ with the quotient topologies, then the natural map is continuous. However there is another topology on $\left|\mathfrak{A}^{\prime}\right|$ by viewing it as a subset of $|\mathfrak{A}|$. We denote this topology by $\left\|\mathfrak{A}^{\prime}\right\|$ and call it the subspace topology. In most cases, the quotient topology is strictly stronger than the subspace topology. Hence it is necessary to distinguish the two different topologies.

7.5. Shrinking good coordinate systems. In this subsection we prove Theorem 7.28. First we show that by precompact shrinkings one can make the relation $\vee$ an equivalence relation.

Lemma 7.30. Let $\mathfrak{A}$ be a virtual orbifold atlas on $X$ with the collection of footprints $\left\{F_{I} \mid I \in \mathcal{I}\right\}$. Let $F_{I}^{\square} \sqsubset F_{I}$ be precompact open subsets such that

$$
X=\bigcup_{I \in \mathcal{I}} F_{I}^{\square} .
$$

Then there exists a precompact shrinking $\mathfrak{A}^{\prime}$ of $\mathfrak{A}$ whose collection of footprints $F_{I}^{\prime}$ contains $\overline{F_{I}^{\square}}$ for all $I \in \mathcal{I}$ such that the relation $\vee$ on $\mathfrak{A}^{\prime}$ is an equivalence relation.

Proof. By definition, the relation $\vee$ is reflexive and symmetric. By the comments above, any shrinking of $\mathfrak{A}$ will preserve reflexiveness and symmetry. Hence we only need to shrink the atlas to make the induced relation transitive. 
For any subset $\mathcal{I}^{\prime} \in \mathcal{I}$, we say that $\vee$ is transitive in $\mathcal{I}^{\prime}$ if for $x, y, z \in \bigsqcup_{I \in \mathcal{I}^{\prime}} U_{I}, x \vee y$, $y \vee z$ imply $x \vee z$. Being transitive in any subset $\mathcal{I}^{\prime}$ is a condition that is preserved under shrinking. Hence it suffices to construct shrinkings such that $\checkmark$ is transitive in $\{I, J, K\}$ for any three distinct elements $I, J, K \in \mathcal{I}$. Let $x \in U_{I}, y \in U_{J}, z \in U_{K}$ be general elements.

Since $U_{I}$ (resp. $U_{J}$ resp. $U_{K}$ ) is an orbifold and hence metrizable, we can choose a sequence of precompact open subsets $U_{I}^{n} \sqsubset U_{I}$ (resp. $U_{J}^{n} \sqsubset U_{J}$ resp. $\left.U_{K}^{n} \sqsubset U_{K}\right)$ containing $\overline{F_{I}^{\square}}$ (resp. $\overline{F_{J}^{\square}}$ resp. $\overline{F_{K}^{\square}}$ ) such that

$$
U_{I}^{n+1} \sqsubset U_{I}^{n}, \quad U_{J}^{n+1} \sqsubset U_{J}^{n}, \quad U_{K}^{n+1} \sqsubset U_{K}^{n},
$$

and

$$
\bigcap_{n} U_{I}^{n}=\psi_{I}^{-1}\left(\overline{F_{I}^{\square}}\right), \quad \bigcap_{n} U_{J}^{n}=\psi_{J}^{-1}\left(\overline{F_{J}^{\square}}\right), \quad \bigcap_{n} U_{K}^{n}=\psi_{K}^{-1}\left(\overline{F_{K}^{\square}}\right) .
$$

Then for each $n, U_{I}^{n}, U_{J}^{n}, U_{K}^{n}$ induce a shrinking of the atlas $\mathfrak{A}$, denoted by $\mathfrak{A}^{n}$. Let the induced binary relation on $U_{I}^{n} \sqcup U_{J}^{n} \sqcup U_{K}^{n}$ still by $\vee$. We claim that for $n$ large enough, $\checkmark$ is an equivalence relation on this triple disjoint union. Denote the domains of the shrunk coordinate changes by $U_{J I}^{n}, U_{K J}^{n}$ and $U_{K I}^{n}$ respectively.

If this is not true, then without loss of generality, we may assume that for all large $n$, there exist points $x^{n} \in U_{I}^{n}, y^{n} \in U_{J}^{n}, z^{n} \in U_{K}^{n}$ such that

$$
x^{n} \vee y^{n}, y^{n} \vee z^{n} \text {, but }\left(x^{n}, z^{n}\right) \notin \vee ;
$$

Then for some subsequence (still indexed by $n$ ), $x^{n}, y^{n}$ and $z^{n}$ converge to $x^{\infty} \epsilon$ $\psi_{I}^{-1}\left(\overline{F_{I}^{\square}}\right) \subset U_{I}, y^{\infty} \in \psi_{J}^{-1}\left(\overline{F_{J}^{\square}}\right) \subset U_{J}$ and $z^{\infty} \in \psi_{K}^{-1}\left(\overline{F_{K}^{\square}}\right) \subset U_{K}$ respectively. Then by the definition of coordinate changes (Definition 7.22), one has

$$
x^{\infty} \vee y^{\infty}, y^{\infty} \vee z^{\infty} \Longrightarrow \psi_{I}\left(x^{\infty}\right)=\psi_{J}\left(y^{\infty}\right)=\psi_{K}\left(z^{\infty}\right) \in \overline{F_{I}^{\square}} \cap \overline{F_{J}^{\square}} \cap \overline{F_{K}^{\square}} .
$$

By the (Overlapping Condition) of Definition 7.24, $\{I, J, K\}$ is totally ordered. Since the roles of $K$ and $I$ are symmetric, we may assume that $I \leqslant K$. Then since

$$
x^{\infty} \in \psi_{I}^{-1}\left(\overline{F_{I}^{\square}} \cap \overline{F_{K}^{\square}}\right) \subset \psi_{I}^{-1}\left(F_{I} \cap F_{K}\right)=\psi_{I}^{-1}\left(F_{K I}\right) \subset U_{K I}
$$

and $U_{K I} \subset U_{I}$ is an open set, for $n$ large enough one has

$$
x^{n} \in U_{K I} .
$$

(1) If $I \leqslant J \leqslant K$, then by (Cocycle Condition) of Definition 7.24,

$$
\phi_{K I}\left(x^{n}\right)=\phi_{K J}\left(\phi_{J I}\left(x^{n}\right)\right)=\phi_{K J}\left(y^{n}\right)=z^{n} .
$$

So $x^{n} \vee z^{n}$, which contradicts (7.4).

(2) If $J \leqslant I \leqslant K$, then (Cocycle Condition) of Definition 7.24,

$$
z^{n}=\phi_{K J}\left(y^{n}\right)=\phi_{K I}\left(\phi_{I J}\left(y^{n}\right)\right)=\phi_{K I}\left(x^{n}\right) .
$$

So $x^{n} \vee z^{n}$, which contradicts $(7.4)$.

(3) If $I \leqslant K \leqslant J$, then since $\phi_{K I}\left(x^{\infty}\right) \in \psi_{K}^{-1}\left(F_{J} \cap F_{K}\right) \subset U_{J K}$, for large $n, x^{n} \in$ $\phi_{K I}^{-1}\left(U_{J K}\right)$. Then by (Cocycle Condition) of Definition 7.24,

$$
\phi_{J K}\left(z^{n}\right)=y^{n}=\phi_{J I}\left(x^{n}\right)=\phi_{J K}\left(\phi_{K I}\left(x^{n}\right)\right) \text {. }
$$

Since $\phi_{J K}$ is an embedding, we have $\phi_{K I}\left(x^{n}\right)=z^{n}$. Therefore, $x^{n} \vee z^{n}$, which contradicts $(7.4)$. 
Therefore, $\vee^{n}$ is an equivalence relation on $U_{I}^{n} \sqcup U_{J}^{n} \sqcup U_{K}^{n}$ for large enough $n$. We can perform the shrinking for any triple of elements of $\mathcal{I}$, which eventually makes $\checkmark$ an equivalence relation. By the construction the shrunk footprints $F_{I}^{\prime}$ still contain $\overline{F_{I}^{\square}}$.

Lemma 7.31. Suppose $\mathfrak{A}$ is a virtual orbifold atlas on $X$ such that the relation $\vee$ is an equivalence relation. Suppose there is a collection of precompact subsets $F_{I}^{\square} \sqsubset F_{I}$ of footprints of $\mathfrak{A}$ such that

$$
X=\bigcup_{I \in \mathcal{I}} F_{I}^{\square}
$$

Then there exists a precompact shrinking $\mathfrak{A}^{\prime} \sqsubset \mathfrak{A}$ satisfying

(1) The shrunk footprints $F_{I}^{\prime}$ all contain $F_{I}^{\square}$.

(2) The virtual neighborhood $\left|\mathfrak{A}^{\prime}\right|$ is a Hausdorff space.

Before proving Lemma 7.31, we need some preparations. Order the finite set $\mathcal{I}$ as $\left\{I_{1}, \ldots, I_{m}\right\}$ such that for $k=1, \ldots, m$,

$$
I_{k} \leqslant J \Longrightarrow J \in\left\{I_{k}, I_{k+1}, \ldots, I_{m}\right\} .
$$

For each $k, \vee$ induces an equivalence relation on $\bigsqcup_{i \geq k} U_{I_{i}}$ and denote the quotient space by $\left|\mathfrak{A}_{k}\right|$. Then the map $\pi_{\mathfrak{A}}$ of (7.3) induces a natural continuous map

$$
\pi_{k}: \bigsqcup_{i \geq k} U_{I_{i}} \rightarrow\left|\mathfrak{A}_{k}\right| \text {. }
$$

Lemma 7.32. For $k=1, \ldots, m$, if $\left|\mathfrak{A}_{k}\right|$ is Hausdorff and $\mathfrak{A}^{\prime}$ is a shrinking of $\mathfrak{A}$, then $\left|\mathfrak{A}_{k}^{\prime}\right|$ is also Hausdorff.

Proof. Left to the reader. A general fact is that the quotient topology is always stronger than (or homeomorphic to) the subspace topology (see Remark 7.29).

Lemma 7.33. The natural map

$$
\left|\mathfrak{A}_{k+1}\right| \rightarrow\left|\mathfrak{A}_{k}\right|
$$

is a homeomorphism onto an open subset.

Proof. The map is clearly continuous and injective. To show that it is a homeomorphism onto an open set, consider any open subset $O_{k+1}$ of its domain. Its preimage under the quotient map

is denoted by

$$
U_{I_{k+1}} \sqcup \cdots \sqcup U_{I_{m}} \rightarrow\left|\mathfrak{A}_{k+1}\right|
$$

$$
\tilde{O}_{k+1}=O_{I_{k+1}} \sqcup \cdots \sqcup O_{I_{m}}
$$

where $O_{I_{k+1}}, \ldots, O_{I_{m}}$ are open subsets of $U_{I_{k+1}}, \ldots, U_{I_{m}}$ respectively. Define

$$
O_{I_{k}}:=\bigcup_{i \geq k+1, I_{i} \geq I_{k}} \phi_{I_{i} I_{k}}^{-1}\left(O_{I_{i}}\right) \text {. }
$$

This is an open subset of $U_{I_{k}}$. Then the image of $O_{k+1}$ under the map (7.5), denoted by $O_{k}$, is the quotient of

$$
\tilde{O}_{k}:=O_{I_{k}} \sqcup O_{I_{k+1}} \sqcup \cdots \sqcup O_{I_{m}} \subset U_{I_{k}} \sqcup \cdots \sqcup U_{I_{m}}
$$

On the other hand, $\tilde{O}_{k}$ is exactly the preimage of $O_{k}$ under the quotient map. Hence by the definition of the quotient topology, $O_{k}$ is open. This show that (7.5) is a homeomorphism onto an open subset. 
Proof of Lemma 7.31 . For each $k$, we would like to construct shrinkings $U_{I_{i}}^{\prime} \sqsubset U_{I_{i}}$ for all $i \geq k$ such that $\left|\mathfrak{A}_{k}^{\prime}\right|$ is Hausdorff and the shrunk footprints $F_{I_{i}}^{\prime}$ contains $\overline{F_{I_{i}}^{\square}}$ for all $i \geq k$. Our construction is based on a top-down induction. First, for $k=m,\left|\mathfrak{A}_{m}\right| \simeq U_{I_{m}}$ and hence is Hausdorff. Suppose after shrinking $\left|\mathfrak{A}_{k+1}\right|$ is already Hausdorff.

Choose open subsets $F_{I_{i}}^{\prime} \sqsubset F_{I_{i}}$ for all $i \geq k$ such that

$$
\overline{F_{I_{i}}^{\square}} \subset F_{I_{i}}^{\prime}, \quad X=\bigcup_{i \geq k} F_{I_{i}}^{\prime} \cup \bigcup_{i \leq k-1} F_{I_{i}} .
$$

Choose precompact open subsets $U_{I_{i}}^{\prime} \sqsubset U_{I_{i}}$ for all $i \geq k$ such that

$$
\psi_{I_{i}}\left(U_{I_{i}}^{\prime} \cap S_{I_{i}}^{-1}(0)\right)=F_{I_{i}}^{\prime}, \quad \psi_{I_{i}}\left(\overline{U_{I_{i}}^{\prime}} \cap S_{I_{i}}^{-1}(0)\right)=\overline{F_{I_{i}}^{\prime}} .
$$

Then $U_{I_{i}}^{\prime}$ for $i \geq k$ and $U_{I_{i}}$ for $i<k$ provide a shrinking $\mathfrak{A}^{\prime}$ of $\mathfrak{A}$. We claim that $\left|\mathfrak{A}_{k}^{\prime}\right|$ is Hausdorff.

Indeed, pick any two different points $|x|,|y| \in\left|\mathfrak{A}_{k}^{\prime}\right|$. If $|x|,|y| \in\left|\mathfrak{A}_{k+1}^{\prime}\right| \subset\left|\mathfrak{A}_{k}^{\prime}\right|$, then by the induction hypothesis and Lemma 7.32, $|x|$ and $|y|$ can be separated by two open subsets in $\left|\mathfrak{A}_{k+1}^{\prime}\right|$. Then by Lemma 7.33 , these two open sets are also open sets in $\left|\mathfrak{A}_{k}^{\prime}\right|$. Hence we assume that one or both of $|x|$ and $|y|$ are in $\left|\mathfrak{A}_{k}^{\prime}\right| \backslash\left|\mathfrak{A}_{k+1}^{\prime}\right|$.

Case 1. Suppose $|x|$ and $|y|$ are represented by $x, y \in U_{I_{k}}^{\prime}$. Choose a distance function on $U_{I_{k}}$ which induces the same topology. Let $O_{x}^{\epsilon}$ and $O_{y}^{\epsilon}$ be the open $\epsilon$-balls in $U_{I_{k}}^{\prime}$ centered at $x$ and $y$ respectively. Then for $\epsilon$ small enough, $\overline{O_{x}^{\epsilon}} \cap \overline{O_{y}^{\epsilon}}=\varnothing$.

Claim. For $\epsilon$ sufficiently small, for all $I_{k} \leqslant I_{i}$ and $I_{k} \leqslant I_{j}$, one has

$$
\pi_{k+1}\left(\overline{\phi_{I_{i} I_{k}}\left(O_{x}^{\epsilon} \cap U_{I_{i} I_{k}}^{\prime}\right)}\right) \cap \pi_{k+1}\left(\overline{\phi_{I_{j} I_{k}}\left(O_{y}^{\epsilon} \cap U_{I_{j} I_{k}}^{\prime}\right)}\right)=\varnothing .
$$

Here the closures are the closures in $U_{I_{i}}$ and $U_{I_{j}}$ respectively.

Proof of the claim. Suppose it is not the case, then there exist a sequence $\epsilon_{n} \rightarrow 0, I_{k} \leqslant I_{i}$, $I_{k} \leqslant I_{j}$, and a sequence of points

$$
\left|z^{n}\right| \in \pi_{k+1}\left(\overline{\phi_{I_{i} I_{k}}\left(O_{x}^{\epsilon_{n}} \cap U_{I_{i} I_{k}}^{\prime}\right)}\right) \cap \pi_{k+1}\left(\overline{\phi_{I_{j} I_{k}}\left(O_{y}^{\epsilon} \cap U_{I_{j} I_{k}}^{\prime}\right)}\right) \subset\left|\mathfrak{A}_{k+1}\right| .
$$

Then $\left|z^{n}\right|$ has its representative $p^{n} \in \overline{\phi_{I_{i} I_{k}}\left(O_{x}^{\epsilon_{n}} \cap U_{I_{k} I_{i}}^{\prime}\right)} \subset U_{I_{i}}$ and its representative $q^{n} \epsilon$ $\overline{\phi_{I_{j} I_{k}}\left(O_{y}^{\epsilon_{n}} \cap U_{I_{j} I_{k}}^{\prime}\right)} \subset U_{I_{j}}$. Then $p^{n} \vee q^{n}$ and without loss of generality, assume that $I_{i} \leqslant I_{j}$. Then $p^{n} \in U_{I_{j} I_{i}}$ and $q^{n}=\phi_{I_{j} I_{i}}\left(p^{n}\right)$.

Choose distance functions $d_{i}$ on $U_{I_{i}}$ and $d_{j}$ on $U_{I_{j}}$ which induce the same topologies. Then one can choose $x^{n} \in O_{x}^{\epsilon_{n}} \cap U_{I_{i} I_{k}}^{\prime}$ and $y^{n} \in O_{y}^{\epsilon_{n}} \cap U_{I_{j} I_{k}}^{\prime}$ such that

$$
d_{i}\left(p^{n}, \phi_{I_{i} I_{k}}\left(x^{n}\right)\right) \leq \epsilon_{n}, \quad d\left(q^{n}, \phi_{I_{i} I_{k}}\left(y^{n}\right)\right) \leq \epsilon_{n} .
$$

Since $\overline{U_{I_{i}}^{\prime}}$ and $\overline{U_{I_{j}}^{\prime}}$ are compact and $p^{n} \in \overline{U_{I_{i}}^{\prime}}, q^{n} \in \overline{U_{I_{j}}^{\prime}}$, for some subsequence (still indexed by $n), p^{n}$ converges to some $p^{\infty} \in \overline{U_{I_{i}}^{\prime}}$ and $q^{n}$ converges to some $q^{\infty} \in \overline{U_{I_{j}}^{\prime}}$. Then $p^{\infty} \vee q^{\infty}$. Moreover, by (7.6), one has

$$
\lim _{n \rightarrow \infty} \phi_{I_{i} I_{k}}\left(x^{n}\right)=p^{\infty}, \quad \quad \lim _{n \rightarrow \infty} \phi_{I_{j} I_{k}}\left(y^{n}\right)=q^{\infty} .
$$

On the other hand, $x^{n}$ converges to $x$ and $y^{n}$ converges to $y$. By the property of coordinate changes, one has that $x \in U_{I_{i} I_{k}}, y \in U_{I_{j} I_{k}}$ and

$$
x \vee p^{\infty} \vee q^{\infty} \vee y .
$$


Since $\checkmark$ is an equivalence relation and it remains an equivalence relation after shrinking, $x \vee y$, which contradicts $x \neq y$.

End of the proof of the claim.

Now choose such an $\epsilon$ and abbreviate $O_{x}=O_{x}^{\epsilon}, O_{y}=O_{y}^{\epsilon}$. Denote

$$
P_{I_{i}}=\overline{\phi_{I_{i} I_{k}}\left(O_{x} \cap U_{I_{i} I_{k}}^{\prime}\right)} \subset \overline{U_{I_{i}}^{\prime}}, \quad Q_{I_{i}}=\overline{\phi_{I_{i} I_{k}}\left(O_{y} \cap U_{I_{i} I_{k}}^{\prime}\right)} \subset \overline{U_{I_{i}}^{\prime}}
$$

(which could be empty). They are all compact, hence

$$
P_{k+1}:=\pi_{k+1}\left(\bigsqcup_{i \geq k+1} P_{I_{i}}\right) \subset\left|\mathfrak{A}_{k+1}\right|, \quad Q_{k+1}:=\pi_{k+1}\left(\bigsqcup_{i \geq k+1} Q_{I_{i}}\right) \subset\left|\mathfrak{A}_{k+1}\right|
$$

are both compact. The above claim implies that $P_{k+1} \cap Q_{k+1}=\varnothing$. Then by the induction hypothesis which says that $\left|\mathfrak{A}_{k+1}\right|$ is Hausdorff, they can be separated by open sets $V_{k+1}, W_{k+1} \subset\left|\mathfrak{A}_{k+1}\right|$. Write

$$
\pi_{k+1}^{-1}\left(V_{k+1}\right)=\bigsqcup_{i \geq k+1} V_{I_{i}}, \quad \pi_{k+1}^{-1}\left(W_{k+1}\right)=\bigsqcup_{i \geq k+1} W_{I_{i}} .
$$

Define $V_{I_{i}}^{\prime}=V_{I_{i}} \cap U_{I_{i}}^{\prime}, W_{I_{i}}^{\prime}=W_{I_{i}} \cap U_{I_{i}}^{\prime}$ and

$$
V_{I_{k}}^{\prime}:=O_{x} \cup \bigcup_{I_{k} \leqslant I_{i}} \phi_{I_{i} I_{k}}^{-1}\left(V_{I_{i}}^{\prime}\right) \cap U_{I_{k}}^{\prime}, \quad W_{I_{k}}^{\prime}:=O_{y} \cup \bigcup_{I_{k} \leqslant I_{i}} \phi_{I_{i} I_{k}}^{-1}\left(W_{I_{i}}^{\prime}\right) \cap U_{I_{k}}^{\prime}
$$

and

$$
V_{k}^{\prime}:=\pi_{k}\left(\bigsqcup_{i \geq k} V_{I_{i}}^{\prime}\right) \subset\left|\mathfrak{A}_{k}^{\prime}\right|, \quad \quad W_{k}^{\prime}:=\pi_{k}\left(\bigsqcup_{i \geq k} W_{I_{i}}^{\prime}\right) \subset\left|\mathfrak{A}_{k}^{\prime}\right| .
$$

It is easy to check that $V_{k}^{\prime}$ and $W_{k}^{\prime}$ are disjoint open subsets containing $|x|$ and $|y|$ respectively. Therefore $|x|$ and $|y|$ are separated in $\left|\mathfrak{A}_{k}^{\prime}\right|$.

Case 2. Now suppose $|x|$ is represented by $x \in U_{I_{k}}^{\prime}$ and $|y| \in\left|\mathfrak{A}_{k+1}^{\prime}\right|$. Similar to Case 1, we claim that for $\epsilon$ sufficiently small, for all $I_{k} \leqslant I_{i}$, one has

$$
|y| \notin \pi_{k+1}\left(\overline{\phi_{I_{i} I_{k}}\left(O_{x}^{\epsilon}\right) \cap U_{I_{i} I_{k}}^{\prime}}\right)=: P_{I_{i}} \subset \overline{U_{I_{i}}^{\prime}} \text {. }
$$

The proof is similar and is omitted. Then choose such an $\epsilon$ and abbreviate $O_{x}=O_{x}^{\epsilon}$. $P_{I_{i}}$ are compact sets and so is

$$
P_{k+1}:=\pi_{k+1}\left(\bigsqcup_{i \geq k+1} P_{I_{i}}\right) \subset\left|\mathfrak{A}_{k+1}\right| .
$$

The above claim implies that $|y| \notin P_{k+1}$. Then by the induction hypothesis, $|y|$ and $P_{k+1}$ can be separated by open sets $V_{k+1}$ and $W_{k+1}$ of $\left|\mathfrak{A}_{k+1}\right|$. By similar procedure as in Case 1 above, one can produce two open subsets of $\left|\mathfrak{A}_{k}^{\prime}\right|$ which separate $|x|$ and $|y|$.

Therefore, we can finish the induction and construct a shrinking such that $\left|\mathfrak{A}^{\prime}\right|$ is Hausdorff. Eventually the shrunk footprints still contain $\overline{F_{I}^{\square}}$.

Now we can finish proving Theorem 7.28. Suppose $|\mathfrak{A}|$ is Hausdorff. By the definition of the quotient topology, the natural map $U_{I} \hookrightarrow|\mathfrak{A}|$ is continuous. Since $U_{I}$ is locally compact and $|\mathfrak{A}|$ is Hausdorff, a further shrinking can make this map a homeomorphism onto its image. This uses the fact that a continuous bijection from a compact space to a Hausdorff space is necessarily a homeomorphism. Hence the third condition for a good coordinate system is satisfied by a precompact shrinking of $\mathfrak{A}$, and this condition is preserved for any further shrinking. This establishes Theorem 7.28. 
7.6. Perturbations. Now we define the notion of perturbations.

Definition 7.34. Let $\mathfrak{A}$ be a good coordinate system on $X$.

(1) A multi-valued perturbation of $\mathfrak{A}$, simply called a perturbation, denoted by $\mathfrak{t}$, consists of a collection of multi-valued continuous sections

$$
t_{I}: U_{I} \stackrel{m}{\rightarrow} E_{I}
$$

satisfying (as multisections)

$$
t_{J} \circ \phi_{J I}=\left.\widehat{\phi}_{J I} \circ t_{I}\right|_{U_{J I}}
$$

(2) Given a multi-valued perturbation $\mathfrak{t}$, the object

$$
\tilde{\mathfrak{s}}=\left(\tilde{s}_{I}=S_{I}+t_{I}: U_{I} \stackrel{m}{\rightarrow} E_{I}\right)
$$

satisfies the same compatibility condition with respect to coordinate changes. The perturbation $\mathfrak{t}$ is called transverse if every $\tilde{s}_{I}$ is a transverse multisection.

(3) Suppose $\mathfrak{A}$ is thickened by $\mathcal{N}=\left\{\left(N_{J I}, E_{I ; J}\right) \mid I \leqslant J\right\}$. We say that $\mathfrak{t}$ is $\mathcal{N}$-normal if for all $I \leqslant J$, one has

$$
\left.t_{J}\left(N_{J I}\right) \subset E_{I ; J}\right|_{N_{J I}} .
$$

(4) The zero locus of a perturbed $\tilde{\mathfrak{s}}$ gives objects in various different categories. Denote

$$
\mathcal{Z}=\bigsqcup_{I \in \mathcal{I}} \tilde{s}_{I}^{-1}(0)
$$

It is naturally equipped with the topology induced from the disjoint union of $U_{I}$. Denote by

$$
|\mathcal{Z}|:=\mathcal{Z} / \vee
$$

the quotient of $\mathcal{Z}$, which is equipped with the quotient topology. Furthermore, there is a natural injection $|\mathcal{Z}| \hookrightarrow|\mathfrak{A}|$. Denote by $\|\mathcal{Z}\|$ the same set as $|\mathcal{Z}|$ but equipped with the topology as a subspace of $|\mathfrak{A}|$.

In order to construct suitable perturbations of a good coordinate system, we need certain tubular neighborhood structures with respect to coordinate changes. In our topological situation, it is sufficient to have some weaker structure near the embedding images.

Definition 7.35. Let $\mathfrak{A}$ be a good coordinate system with charts indexed by elements in a finite partially ordered set $(\mathcal{I}, \leqslant)$ and coordinate changes indexed by pairs $I \leqslant J \in \mathcal{I}$. A thickening of $\mathfrak{A}$ is a collection of objects

$$
\left\{\left(N_{J I}, E_{I ; J}\right) \mid I \leqslant J\right\}
$$

where $N_{J I} \subset U_{J}$ is an open neighborhood of $\phi_{J I}\left(U_{J I}\right)$ and $E_{I ; J}$ is a subbundle of $\left.E_{J}\right|_{N_{J I}}$. They are required to satisfy the following conditions.

(1) If $I \leqslant K, J \leqslant K$ but there is no partial order relation between $I$ and $J$, then

$$
N_{K I} \cap N_{K J}=\varnothing \text {. }
$$

(2) For all triples $I \leqslant J \leqslant K$,

$$
\left.E_{I ; J}\right|_{\phi_{K J}^{-1}\left(N_{K I}\right) \cap N_{J I}}=\left.\widehat{\phi}_{K J}^{-1}\left(E_{I ; K}\right)\right|_{\phi_{K J}^{-1}\left(N_{K I}\right) \cap N_{J I}} .
$$


(3) For all triples $I \leqslant J \leqslant K$, one has

$$
\left.\left.E_{I ; K}\right|_{N_{K I} \cap N_{K J}} \subset E_{J ; K}\right|_{N_{K I} \cap N_{K J}} .
$$

(4) Each $\left(N_{J I}, E_{I ; J}\right)$ satisfies the (Tangent Bundle Condition) of Definition 7.20.

Remark 7.36. The above setting is slightly more general than what we need in our application in this paper and the companion [TX]. In this paper we will see the following situation in the concrete cases.

(1) The index set $\mathcal{I}$ consists of certain nonempty subsets of a finite set $\{1, \ldots, m\}$, which has a natural partial order given by inclusions.

(2) For each $i \in I, \Gamma_{i}$ is a finite group and $\Gamma_{I}=\Pi_{i \in I} \Gamma_{i} . U_{I}=\tilde{U}_{I} / \Gamma_{I}$ where $\tilde{U}_{I}$ is a topological manifold acted by $\Gamma_{I}$. Moreover, $\boldsymbol{E}_{1}, \ldots, \boldsymbol{E}_{m}$ are vector spaces acted by $\Gamma_{i}$ and the orbifold bundle $E_{I} \rightarrow U_{I}$ is the quotient

$$
E_{I}:=\left(\tilde{U}_{I} \times \boldsymbol{E}_{I}\right) / \Gamma_{I} \text {, where } \boldsymbol{E}_{I}:=\bigoplus_{i \in I} \boldsymbol{E}_{i} .
$$

(3) For $I \leqslant J, U_{J I}=\tilde{U}_{J I} / \Gamma_{I}$ where $\tilde{U}_{J I} \subset \tilde{U}_{I}$ is a $\Gamma_{I}$-invariant open subset and the coordinate change is induced from the following diagram

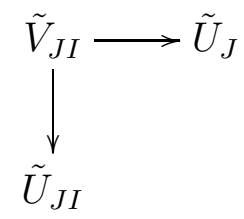

Here $\tilde{V}_{J I} \rightarrow \tilde{U}_{J I}$ is a covering space with group of deck transformations identical to $\Gamma_{J-I}=\Pi_{j \in J-I} \Gamma_{j}$; then $\Gamma_{J}$ acts on $\tilde{V}_{J I}$ and $\tilde{V}_{J I} \rightarrow \tilde{U}_{J}$ is a $\Gamma_{J}$-equivariant embedding of manifolds, which induces an orbifold embedding $U_{J I} \rightarrow U_{J}$ and an orbibundle embedding $\left.E_{I}\right|_{U_{J I}} \rightarrow E_{J}$.

In this situation, one naturally has subbundles $E_{I ; J} \subset E_{J}$ for all pairs $I \leqslant J$. Hence a thickening of such a good coordinate system is essentially only a collection of neighborhoods $N_{J I}$ of $\phi_{J I}\left(U_{J I}\right)$ which satisfy $(7.8)$ and

$$
S_{J}^{-1}\left(E_{I ; J}\right) \cap N_{J I}=\phi_{J I}\left(U_{J I}\right) .
$$

Theorem 7.37. Let $X$ be a compact Hausdorff space and have a good coordinate system

$$
\mathfrak{A}=\left(\left\{C_{I}=\left(U_{I}, E_{I}, S_{I}, \psi_{I}, F_{I}\right) \mid I \in \mathcal{I}\right\},\left\{T_{J I} \mid I \leqslant J\right\}\right) .
$$

Let $\mathfrak{A}^{\prime} \sqsubset \mathfrak{A}$ be any precompact shrinking. Let $\mathcal{N}=\left\{\left(N_{J I}, E_{I ; J}\right)\right\}$ be a thickening of $\mathfrak{A}$ and $N_{J I}^{\prime} \subset U_{J}^{\prime}$ be a collection of open neighborhoods of $\phi_{J I}\left(U_{J I}^{\prime}\right)$ such that $\overline{N_{J I}^{\prime}} \subset N_{J I}$. Then they induce a thickening $\mathcal{N}^{\prime}$ of $\mathfrak{A}^{\prime}$ by restriction. Let $d_{I}: U_{I} \times U_{I} \rightarrow[0,+\infty)$ be a distance function on $U_{I}$ which induces the same topology as $U_{I}$. Let $\epsilon>0$ be a constant. Then there exist a collection of multisections $t_{I}: U_{I} \rightarrow E_{I}$ satisfying the following conditions.

(1) For each $I \in \mathcal{I}, \tilde{s}_{I}:=S_{I}+t_{I}$ is transverse.

(2) For each $I \in \mathcal{I}$,

$$
d_{I}\left(\tilde{s}_{I}^{-1}(0) \cap \overline{U_{I}^{\prime}}, S_{I}^{-1}(0) \cap \overline{U_{I}^{\prime}}\right) \leq \epsilon .
$$


(3) For each pair $I \leqslant J$, we have

$$
\left.\widehat{\phi}_{J I} \circ t_{I}\right|_{U_{J I}^{\prime}}=\left.t_{J} \circ \phi_{J I}\right|_{U_{J I}^{\prime}} .
$$

Hence the collection of restrictions $t_{I}^{\prime}:=\left.t_{I}\right|_{U_{I}^{\prime}}$ defines a perturbation $\mathfrak{t}^{\prime}$ of $\mathfrak{A}^{\prime}$.

(4) $\mathfrak{t}^{\prime}$ is $\mathcal{N}^{\prime}$-normal.

Proof. To simplify the proof, we assume that we are in the situation described by Remark 7.36. The general case requires minor modifications in a few places. Then the subbundles $E_{I_{b}, I_{a}}$ are naturally define over $U_{I_{b}}$.

We use the inductive construction. Order the set $\mathcal{I}$ as $I_{1}, \ldots, I_{m}$ such that

$$
I_{k} \leqslant I_{l} \Longrightarrow k \leq l \text {. }
$$

For each $k, l$ with $k<l$, define open sets $N_{I_{l}, k}^{-}$by

$$
N_{I_{l}, k}^{-}=\bigcup_{a \leq k, I_{a}<I_{l}} N_{I_{l} I_{a}} .
$$

Define open sets $N_{I_{l}, k}^{+}$inductively. First $N_{I_{m}, k}^{+}=\varnothing$. Then

$$
N_{I_{l}, k}^{+}:=\bigcup_{I_{l}<I_{b}} \phi_{I_{b} I_{l}}^{-1}\left(N_{I_{b}, k}\right), \quad \quad N_{I_{l}, k}=N_{I_{l}, k}^{-} \cup N_{I_{l}, k}^{+} .
$$

Replacing $N_{J I}$ by $N_{J I}^{\prime}$ in the above definitions, we obtain $N_{I_{l}, k}^{\prime} \subset U_{I_{l}}^{\prime}$ with

$$
\overline{N_{I_{l}, k}^{\prime}} \subset N_{I_{l}, k}
$$

If $k \geq l$, define

$$
N_{I_{l}, k}=U_{I_{l}}, \quad N_{I_{l}, k}^{\prime}=U_{I_{l}}^{\prime} .
$$

On the other hand, it is not hard to inductively choose a system of continuous norms on $E_{I}$ such that, for all pairs $I_{a} \leqslant I_{b}$, the bundle embedding $\widehat{\phi}_{I_{b} I_{a}}$ is isometric. Given such a collection of norms, choose $\delta>0$ such that for all $I$,

$$
d_{I}\left(x, S_{I}^{-1}(0) \cap \overline{U_{I}^{\prime}}\right)>\epsilon, x \in \overline{U_{I}^{\prime}} \Longrightarrow\left\|S_{I}(x)\right\| \geq(2 m+1) \delta .
$$

Now we reformulate the problem in an inductive fashion. We would like to verify the following induction hypothesis.

Induction Hypothesis. For $a, k=1, \ldots, m$, there exists an open subset $O_{I_{a}, k} \subset N_{I_{a}, k}$ which contains $\overline{N_{I_{a}, k}^{\prime}}$ and multisections

$$
t_{I_{a}, k}: O_{I_{a}, k} \stackrel{m}{\rightarrow} E_{I_{a}} .
$$

They satisfy the following conditions.

(1) For all pairs $I_{a} \leqslant I_{b}$, over a neighborhood of the compact subset

$$
\overline{N_{I_{a}, k}^{\prime}} \cap \phi_{I_{b} I_{a}}^{-1}\left(\overline{N_{I_{b}, k}^{\prime}}\right) \subset \overline{U_{I_{b} I_{a}}^{\prime}} \subset U_{I_{b} I_{a}}
$$

one has

$$
t_{I_{b}, k} \circ \phi_{I_{b} I_{a}}=\widehat{\phi}_{I_{b} I_{a}} \circ t_{I_{a}, k} .
$$

(2) In a neighborhood of $\overline{N_{I_{b} I_{a}}^{\prime}}$, the value of $t_{I_{b}, k}$ is contained in the subbundle $E_{I_{a} ; I_{b}}$.

(3) $S_{I_{a}}+t_{I_{a}, k}$ is transverse.

(4) $\left\|t_{I_{a}, k}\right\|_{C^{0}} \leq 2 k \delta$. 
It is easy to see that the $k=m$ case implies this theorem. Indeed, (7.10) follows from (7.11) and the bound $\left\|t_{I_{l}, m}\right\| \leq m \delta$. Now we verify the conditions of the induction hypothesis. For the base case, apply Lemma 7.17 to

$$
M=U_{I_{1}}, \quad C=\varnothing, \quad D=U_{I_{1}},
$$

we can construct a multisection $t_{I_{1}, 1}: U_{I_{1}} \stackrel{m}{\rightarrow} E_{I_{1}}$ making $S_{I_{1}}+t_{I_{1}}$ transverse with $\left\|t_{I_{1}}\right\| \leq \delta$. Now we construct $t_{I_{a}, 1}$ for $a=2, \ldots, m$ via a backward induction. Define

$$
O_{I_{a}, 1}=N_{I_{a}, 1}=N_{I_{a} I_{1}} \cup \bigcup_{I_{a}<I_{b}} \phi_{I_{b} I_{a}}^{-1}\left(N_{I_{b}, 1}\right), a=1, \ldots, m .
$$

Then (7.12) determines the value of $t_{I_{m}, 1}$ over the set

$$
\phi_{I_{m} I_{1}}\left(U_{I_{m} I_{1}}\right) \subset N_{I_{m}, 1}=N_{I_{m} I_{1}} \text {. }
$$

It is a closed subset of $N_{I_{m}, 1}$, hence one can extend it to a continuous section of $E_{I_{1} ; I_{m}}$ which can be made satisfy the bound

$$
\left\|t_{I_{m}, 1}\right\| \leq\left(1+\frac{1}{m}\right) \delta
$$

Suppose we have constructed $t_{I_{a}, 1}: O_{I_{a}, 1} \stackrel{m}{\rightarrow} E_{I_{a}}$ for all $a \geq l+1$ such that together with $t_{I_{1}, 1}$ they satisfy the induction hypothesis for $k=1$ with the bound

$$
\left\|t_{I_{a}, 1}\right\| \leq\left(1+\frac{m-l}{m}\right) \delta \text {. }
$$

Then we construct $t_{I_{l}, 1}: O_{I_{l}, 1} \stackrel{m}{\rightarrow} E_{I_{l}}$ as follows. Given

$$
z_{I_{l}} \in \phi_{I_{l} I_{1}}\left(U_{I_{l} I_{1}}\right) \cup N_{I_{l}, 1}^{+}=\phi_{I_{l} I_{1}}\left(U_{I_{l} I_{1}}\right) \cup \bigcup_{I_{l}<I_{b}} \phi_{I_{b} I_{l}}^{-1}\left(N_{I_{b}, 1}\right),
$$

if $z_{I_{l}}$ is in the first component, then define $t_{I_{l}, 1}\left(z_{I_{l}}\right)$ by the formula (7.12) for $b=l, a=1$. If $z_{I_{l}} \in N_{I_{b} I_{l}} \cap \phi_{I_{b} I_{l}}^{-1}\left(N_{I_{b}, 1}\right)$ for some $b$, then define

$$
t_{I_{l}, 1}\left(z_{I_{l}}\right)=\widehat{\phi}_{I_{b} I_{l}}^{-1}\left(t_{I_{b}, 1}\left(\phi_{I_{b} I_{l}}\left(z_{I_{l}}\right)\right)\right) .
$$

It is easy to verify using the (Cocycle Condition) that these definitions agree over some closed neighborhood of

$$
\phi_{I_{l} I_{1}}\left(\overline{U_{I_{l} I_{1}}^{\prime}}\right) \cup \bigcup_{I_{l}<I_{b}} \phi_{I_{b} I_{l}}^{-1}\left(\overline{N_{I_{b} I_{l}}^{\prime}}\right) .
$$

Then one can extend it to a continuous multisection of $E_{I_{1} ; I_{l}}$ satisfying the bound

$$
\left\|t_{I_{l}, 1}\right\| \leq\left(1+\frac{m-l+1}{m}\right) \delta .
$$

Then the induction can be carried on and stops until $l=2$, for which one has the bound

$$
\left\|t_{I_{2}, 1}\right\| \leq\left(1+\frac{m-1}{m}\right) \delta \leq 2 \delta .
$$

The transversality of $S_{I_{a}}+t_{I_{a}, 1}$ for $a \geq 2$ follows from the fact that $t_{I_{a}, 1}$ takes value in $E_{I_{1} ; I_{a}}$, the fact that $\left.S_{I_{a}}\right|_{N_{I_{a} I_{1}}}$ intersects with $E_{I_{1} ; I_{a}}$ transversely along $\phi_{I_{a} I_{1}}\left(U_{I_{a} I_{1}}\right)$, and the fact that $S_{I_{1}}+t_{I_{1}, 1}$ is transverse. Hence we have verified the $k=1$ case of the induction hypothesis. 
Suppose we have verified the induction hypothesis for $k-1$. For all $a \leq k-1$, define

$$
O_{I_{a}, k}=O_{I_{a}, k-1}, \quad t_{I_{a}, k}=t_{I_{a}, k-1} .
$$

The induction hypothesis implies that we have a multisection

$$
t_{I_{k}, k-1}: O_{I_{k}, k-1} \stackrel{m}{\rightarrow} E_{I_{k}}
$$

such that $S_{I_{k}}+t_{I_{k}, k-1}$ is transverse and $\left\|t_{I_{k}, k-1}\right\| \leq(2 k-2) \delta$. Then apply Lemma 7.17 , one can obtain a multisection $t_{I_{k}, k}$ defined over a neighborhood of $\overline{U_{I_{k}}^{\prime}}=\overline{N_{I_{k}, k}^{\prime}}$ contained in $U_{I_{k}}=N_{I_{k}, k}$ such that $S_{I_{k}}+t_{I_{k}, k}$ is transverse, $\left\|t_{I_{k}, k}\right\| \leq(2 k-1) \delta$, and $t_{I_{k}, k}=t_{I_{k}, k-1}$ over a neighborhood of $\overline{N_{I_{k}, k-1}^{\prime}}$ which is smaller than $O_{I_{k}, k-1}$. Then by the similar backward induction as before, using the extension property of continuous multi-valued functions, one can construct perturbations with desired properties. The remaining details are left to the reader.

In our argument, condition (7.10) is crucial in establishing the compactness of the perturbed zero locus. In the situation of Theorem 7.37, suppose a perturbation $\mathfrak{t}^{\prime}$ is constructed over the shrinking $\mathfrak{A}^{\prime} \sqsubset \mathfrak{A}^{\prime \prime}$. Then for a further precompact shrinking $\mathfrak{A}^{\prime \prime} \sqsubset \mathfrak{A}^{\prime}$, (7.10) remains true (with $\overline{U_{I}^{\prime}}$ replaced by $\overline{U_{I}^{\prime \prime}}$ ).

Proposition 7.38. Let $\mathfrak{A}$ be a good coordinate system on $X$ and let $\mathfrak{A}^{\prime} \sqsubset \mathfrak{A}$ be a precompact shrinking. Let $\mathcal{N}$ be a thickening of $\mathfrak{A}$. Equip each chart $U_{I}$ a distance function $d_{I}$ which induces the same topology. Then there exists $\epsilon>0$ satisfying the following conditions. Let $\mathfrak{t}$ be a multi-valued perturbation of $\mathfrak{s}$ which is $\mathcal{N}$-normal. Suppose

$$
d_{I}\left(\tilde{s}_{I}^{-1}(0) \cap \overline{U_{I}^{\prime}}, S_{I}^{-1}(0) \cap \overline{U_{I}^{\prime}}\right) \leq \epsilon, \forall I \in \mathcal{I} .
$$

Then the zero locus $\left\|\left(\tilde{\mathfrak{s}}^{\prime}\right)^{-1}(0)\right\|$ is sequentially compact with respect to the subspace topology induced from $\left|\mathfrak{A}^{\prime}\right|$.

Proof. Now for each $x \in\left|\mathfrak{A}^{\prime}\right|$, define $I_{x} \in \mathcal{I}$ to be the minimal element for which $x$ can be represented by a point $\tilde{x} \in U_{I_{x}}^{\prime}$.

Claim. Given $I$, there exists $\epsilon>0$ such that, for any perturbations $\tilde{\mathfrak{s}}$ that satisfy (7.13), if $x_{i} \in\left\|\left(\tilde{\mathfrak{s}}^{\prime}\right)^{-1}(0)\right\|$ with $I_{x_{i}}=I$ for all $i$, then $x_{i}$ has a convergent subsequence.

Proof of the claim. Suppose this is not true, then there exist a sequence $\epsilon_{k}>0$ that converge to zero, and a sequence of multi-valued perturbations $\mathfrak{t}_{k}$ satisfying

$$
d_{I}\left(\tilde{s}_{k, J}^{-1}(0) \cap \overline{U_{J}^{\prime}}, S_{J}^{-1}(0) \cap \overline{U_{J}^{\prime}}\right) \leq \epsilon_{k}, \forall J \in \mathcal{I}
$$

and sequences of points $\tilde{x}_{k, i} \in \tilde{s}_{k, I}^{-1}(0) \cap U_{I}^{\prime}$ such that the sequence $\left\{x_{k, i}=\pi_{\mathfrak{A}}\left(\tilde{x}_{k, i}\right)\right\}_{i=1}^{\infty}$ does not have a convergent subsequence. Since $\tilde{x}_{k, i} \in \overline{U_{I}^{\prime}}$ which is a compact subset of $U_{I}$, for all $k$ the sequence $\tilde{x}_{k, i}$ has subsequential limits, denoted by $\tilde{x}_{k} \in \tilde{s}_{k, I}^{-1}(0) \cap \overline{U_{I}^{\prime}}$. Then since $\epsilon_{k} \rightarrow 0$, the sequence $\tilde{x}_{k}$ has a subsequential limit $\tilde{x}_{\infty} \in \overline{U_{I}^{\prime}} \cap S_{I}^{-1}(0)$. Denote

$$
x_{\infty}=\psi_{I}\left(\tilde{x}_{\infty}\right) \in \psi_{I}\left(\overline{U_{I}^{\prime}} \cap S_{I}^{-1}(0)\right)=\overline{F_{I}^{\prime}} \subset F_{I} \subset X .
$$

Since all $F_{I}^{\prime}$ cover $X$, there exists $J \in \mathcal{I}$ such that $x_{\infty} \in F_{J}$. Then by the (Overlapping Condition) of the atlas $\mathfrak{A}^{\prime}$, we have either $I \leqslant J$ or $J \leqslant I$ but $J \neq I$. We claim that the latter is impossible. Indeed, if $x_{\infty}=\psi_{J}\left(\tilde{y}_{\infty}\right)$ with $\tilde{y}_{\infty} \in U_{J}^{\prime}$, then we have $\tilde{y}_{\infty} \in U_{I J}^{\prime}$ and $\tilde{x}_{\infty} \in \varphi_{I J}^{\prime}\left(U_{I J}^{\prime}\right)$. Then for $k$ sufficiently large, we have $\tilde{x}_{k}$ in $N_{J I}$. Fix such a large 
$k$, then for $i$ sufficiently large, we have $\tilde{x}_{k, i} \in N_{J I}$. However, since the perturbation $\mathfrak{t}$ is $\mathcal{N}$-normal, it follows that $\tilde{x}_{k, i} \in \varphi_{I J}^{\prime}\left(U_{I J}^{\prime}\right)$. This contradicts the assumption that $I_{x_{k, i}}=I$. Therefore $I \leqslant J$. Then there is a unique $\tilde{y}_{\infty} \in U_{J}^{\prime} \cap S_{J}^{-1}(0)$ such that $\psi_{J}^{\prime}\left(\tilde{y}_{\infty}\right)=x_{\infty}$ and $\tilde{y}_{\infty}=\varphi_{J I}\left(\tilde{x}_{\infty}\right)$. Then $\tilde{x}_{\infty} \in U_{J I}$. Therefore, for $k$ sufficiently large, we have $\tilde{x}_{k} \in U_{J I}$ and we have the convergence

$$
\tilde{y}_{k}:=\varphi_{J I}\left(\tilde{x}_{k}\right) \rightarrow \tilde{y}_{\infty}
$$

since $\varphi_{J I}$ is continuous. Since $\tilde{y}_{\infty} \in U_{J}^{\prime}$ which is an open subset of $U_{J}$, for $k$ sufficiently large, we have $\tilde{y}_{k} \in U_{J}^{\prime}$. Fix such a large $k$. Then for $i$ sufficiently large, we have

$$
\tilde{x}_{k, i} \in U_{J I} \cap \varphi_{J I}^{-1}\left(U_{J}^{\prime}\right) \cap U_{I}^{\prime} .
$$

Hence we have $\tilde{y}_{k, i}:=\varphi_{J I}^{\prime}\left(\tilde{x}_{k, i}\right) \in U_{J}^{\prime}$ and

$$
\lim _{i \rightarrow \infty} \tilde{y}_{k, i}=\tilde{y}_{k}
$$

Since the map $U_{J}^{\prime} \rightarrow\left|\mathfrak{A}^{\prime}\right|$ is continuous, we have the convergence

$$
\lim _{i \rightarrow \infty} x_{k, i}=\lim _{i \rightarrow \infty} \pi_{\mathfrak{A}^{\prime}}\left(\tilde{y}_{k, i}\right)=\pi_{\mathfrak{A}^{\prime}}\left(\tilde{y}_{\infty}\right) .
$$

This contradicts the assumption that $x_{k, i}$ does not converge for all $k$. Hence the claim is prove.

End of the proof of the claim.

Now for all $I \in \mathcal{I}$, choose the smallest $\epsilon$ such that the condition of the above claim hold. We claim this $\epsilon$ satisfies the condition of this proposition. Indeed, let $\mathfrak{t}$ be such a perturbation and let $x_{k}$ be a sequence of points in $\left\|\left(\tilde{s}^{\prime}\right)^{-1}(0)\right\|$. Then there exists an $I \in \mathcal{I}$ and a subsequence (still indexed by $k$ ) with $I_{x_{k}}=I$. Then by the above claim, $x_{k}$ has a subsequential limit. Therefore $\left\|\left(\tilde{s}^{\prime}\right)^{-1}(0)\right\|$ is sequentially compact.

7.7. The virtual cardinality and invariance. In the application of the virtual technique in this paper, we only consider two situations: 1) the index of the problem is zero; 2) the index of the problem is zero or one and the charts are all manifolds. Then the discussion of the topology of the perturbed zero locus is very simple. In the general situation, for a transverse multi-valued perturbation, the perturbed zero locus has the structure of a weighted branched manifold.

Indeed, let $\mathfrak{A}$ be a good coordinate system of virtual dimension zero and let $\mathfrak{t}$ be a transverse multi-valued perturbation for which the perturbed zero locus is sequentially compact. Let $|x| \in|\mathfrak{A}|$ be a zero. Then over each chart $C_{I}$ which contains a representative $x_{I} \in U_{I}$ of $|x|$, we have a local representative

$$
\tilde{s}_{I}(x)=\left[s_{I}^{1}(x), \ldots, s_{I}^{l}(x)\right] .
$$

The orientation of the atlas provides a number $\epsilon_{i} \in\{1,-1,0\}$, such that if $s_{I}^{i}\left(x_{I}\right) \neq 0$, then $\epsilon_{i}=0$. Then define the multiplicity of this zero $x_{I}$ by

$$
m(|x|)=\frac{1}{l} \sum_{i=1}^{l} \epsilon_{i} .
$$

One can verify that $m\left(x_{I}\right)$ is independent of the local representative, and is independent of the representative $x_{I}$ of $|x|$. Then we define the virtual cardinality of $\mathfrak{A}$ by

$$
\# \mathfrak{A}=\sum_{|x| \in\left|\mathcal{Z}_{\mathfrak{t}}\right|} m(|x|) \in \mathbb{Q} .
$$


The compactness of $\left|\mathcal{Z}_{\mathfrak{t}}\right|$ implies that this sum is finite and hence $\# \mathfrak{A}$ is a finite number. Moreover, if the charts are all manifolds, then transversality can be achieved by singlevalued perturbations. Therefore, in that case, the virtual cardinality is an integer.

Lastly we expect that the virtual cardinality is independent of various choices. This requires to consider virtual dimension one case, and we assume that the charts are all manifolds. Consider a good coordinate system $\mathfrak{A}$ of virtual dimension one with boundary on a space $X$. Then there is a natural closed subset $\partial X \subset X$ and a boundary atlas $\partial \mathfrak{A}$ on $\partial X$ which has virtual dimension zero without boundary. If $\mathfrak{A}$ is oriented, then $\partial \mathfrak{A}$ has an induced orientation.

Proposition 7.39. Let $\mathfrak{A}$ be an oriented good coordinate system of dimension 1 with boundary on a space $X$. Suppose all charts of $\mathfrak{A}$ are manifolds. Then $\#(\partial \mathfrak{A})=0$.

Proof. Over $\partial \mathfrak{A}$ one can find a transverse single-valued perturbation whose perturbed zero locus is a compact zero-dimensional manifold. The counting with signs of the zeroes is equal to \#( $\partial \mathfrak{A})$. Moreover, the topological transversality theorem (Theorem 7.7) allows us to extend the perturbation to a single-valued transverse perturbation on $\mathfrak{A}$, whose perturbed zero locus is still compact. Then the perturbed zero locus of $\mathfrak{A}$ is a compact oriented one-dimensional manifold whose boundary is the perturbed zero locus of $\partial \mathfrak{A}$, with the induced orientation. Hence $\#(\partial \mathfrak{A})=0$.

The general method to prove the virtual cardinality is independent of choices is to construct a good coordinate system on the product $X \times[0,1]$, such that two different systems of choices can be made homotopic to each other. Then the above proposition implies the independence.

\section{REFERENCES}

$\left[\mathrm{CFG}^{+}\right]$Ionut Ciocan-Fontaine, David Favero, Jŕémy Guéré, Bumsig Kim, and Mark Shoemaker, Fundamental factorization of a GLSM, part I: construction, https://arxiv.org/abs/1802.05247.

[CGS00] Kai Cieliebak, Ana Gaio, and Dietmar Salamon, J-holomorphic curves, moment maps, and invariants of Hamiltonian group actions, International Mathematics Research Notices 16 (2000), 831-882.

[CLLL15] Huai-Liang Chang, Jun Li, Wei-Ping Li, and Chiu-Chu Liu, Mixed-spin-p fields of Fermat quintic polynomials, http://arxiv.org/abs/1505.07532, 2015.

[CLW] Bohui Chen, An-Min Li, and Bai-Ling Wang, Virtual neighborhood technique for pseudoholomorphic spheres, https://arxiv.org/abs/1306.3276.

[FH93] Andreas Floer and Helmut Hofer, Coherent orientations for periodic orbit problems in symplectic geometry, Mathematische Zeitschrift 212 (1993), no. 1, 13-38.

[FJR] Huijun Fan, Tyler Jarvis, and Yongbin Ruan, The Witten equation and its virtual fundamental cycle, http://arxiv.org/abs/0712.4025.

[FJR08] - Geometry and analysis of spin equations, Communications on Pure and Applied Mathematics 61 (2008), no. 6, 745-788.

[FJR13] — The Witten equation, mirror symmetry and quantum singularity theory, Annals of Mathematics 178 (2013), 1-106.

[FJR18] - A mathematical theory of gauged linear $\sigma$-model, Geometry and Topology 22 (2018), 235-303.

[FO99] Kenji Fukaya and Kaoru Ono, Arnold conjecture and Gromov-Witten invariants for general symplectic manifolds, Topology 38 (1999), 933-1048. 
[FOOOa] Kenji Fukaya, Yong-Geun Oh, Hiroshi Ohta, and Kaoru Ono, Kuranishi structure, pseudo-holomorphic curve, and virtual fundamental chain: part 1 , http://arxiv.org/abs/1503.07631.

[FOOOb] _ Kuranishi structure, pseudo-holomorphic curve, and virtual fundamental chain: part 2, https://arxiv.org/abs/1704.01848.

[FOOO12] _ Technical details on Kuranishi structure and virtual fundamental chain, https://arxiv.org/abs/1209.4410, 2012.

[FOOO16] _ Shrinking good coordinate systems associated to Kuranishi structures, Journal of Symplectic Geometry 14 (2016), no. 4, 1295-1310.

[FQ90] Michael Freedman and Frank Quinn, Topology of 4-manifolds, Princeton University Press, 1990.

[HV00] Kentaro Hori and Cumrun Vafa, Mirror symmetry, http://arxiv.org/abs/hep-th/0002222, 2000.

[HWZ07] Helmut Hofer, Krzysztof Wysocki, and Eduard Zehnder, A general Fredholm theory. I. A splicing-based differential geometry, Journal of European Mathematical Society 9 (2007), 841876.

[HWZ09a] _ A general Fredholm theory II: implicit function theorems, Geometric and Functional Analysis 19 (2009), 206-293.

[HWZ09b] _ A general Fredholm theory III: Fredholm functors and polyfolds, Geometry and Topology 13 (2009), 2279-2387.

[Joya] Dominic Joyce, Kuranishi homology and Kuranishi cohomology, http://arxiv.org/abs/0707.3572.

[Joyb] - A new definition of Kuranishi space, https://arxiv.org/abs/1409.6908.

[Kis64] James Kister, Microbundles are fibre bundles, Annals of Mathematics 80 (1964), no. 1, 190199.

[KS77] Robion Kirby and Laurence Siebenmann, Foundational essays on topological manifolds, smoothings, and triangulations, Princeton University Press and University of Tokyo Press, 1977.

[LT98a] Jun Li and Gang Tian, Virtual moduli cycles and Gromov-Witten invariants of algebraic varieties, Journal of American Mathematical Society 11 (1998), no. 1, 119-174.

[LT98b] _ , Virtual moduli cycles and Gromov-Witten invariants of general symplectic manifolds, Topics in symplectic 4-manifolds (Irvine, CA, 1996), First International Press Lecture Series., no. I, International Press, Cambridge, MA, 1998, pp. 47-83.

[LT98c] Gang Liu and Gang Tian, Floer homology and Arnold conjecture, Journal of Differential Geometry 49 (1998), 1-74.

[Maz64] Barry Mazur, The method of infinite repetition in pure topology: I, Annals of Mathematics 80 (1964), no. 2, 201-226.

[Mil64] John Milnor, Microbundles Part I, Topology 3 (1964), no. Suppl. 1, 53-80.

[MS04] Dusa McDuff and Dietmar Salamon, J-holomorphic curves and symplectic topology, Colloquium Publications, vol. 52, American Mathematical Society, 2004.

[MT09] Ignasi Mundet i Riera and Gang Tian, A compactification of the moduli space of twisted holomorphic maps, Advances in Mathematics 222 (2009), 1117-1196.

[Mun99] Ignasi Mundet i Riera, Yang-Mills-Higgs theory for symplectic fibrations, Ph.D. thesis, Universidad Autónoma de Madrid, 1999.

[Mun03] _ Hamiltonian Gromov-Witten invariants, Topology 43 (2003), no. 3, 525-553.

[MW15] Dusa McDuff and Katrin Wehrheim, The fundamental class of smooth Kuranishi atlases with trivial isotropy, Journal of Topology and Analysis Published Online (2015), 1-173.

[MW17a] _ Smooth Kuranishi atlases with isotropy, Geometry and Topology 21 (2017), 27252809.

[MW17b] The topology of Kuranishi atlases, Proceedings of London Mathematical Society 115 (2017), no. 3, 221-292.

[Par16] John Pardon, An algebraic approach to virtual fundamental cycles on moduli spaces of pseudoholomorphic curves, Geometry and Topology 20 (2016), 779-1034. 
[Qui82] Frank Quinn, Ends of maps. III. dimension 4 and 5, Journal of Differential Geometry (1982).

[Qui88] _ Topological transversality holds in all dimensions, Bulletin of the American Mathematical Society 18 (1988), 145-148.

[RT95] Yongbin Ruan and Gang Tian, A mathematical theory of quantum cohomology, Journal of Differential Geometry 42 (1995), 259-367.

[RT97] _ Higher genus symplectic invariants and sigma model coupled with gravity, Inventiones Mathematicae 130 (1997), 455-516.

[Sat56] Ichiro Satake, On a generalization of the notion of manifold, Proceedings of National Academy of Sciences 42 (1956), 359-363.

[Sol05] Jake Solomon, The Witten complex for non-Morse functions, preprint, 2005.

[SX14] Stephen Schecter and Guangbo Xu, Morse theory for Lagrange multipliers and adiabatic limits, Journal of Differential Equations 257 (2014), 4277-4318.

[TX] Gang Tian and Guangbo Xu, A wall-crossing formula for the correlation function of gauged linear $\sigma$-model, preprint.

[TX2] _ Gauged linear sigma model in geometric phases, https://arxiv.org/abs/1809.00424, 2.

[TX15] _ Analysis of gauged Witten equation, Journal für die reine und angewandte Mathematik Published Online (2015), 1-88.

[TX16] Correlation functions in gauged linear $\sigma$-model, Science China. Mathematics 59 (2016), 823-838.

[TX17] , The symplectic approach of gauged linear $\sigma$-model, Proceedings of the Gökova Geometry-Topology Conference 2016 (Selman Akbulut, Denis Auroux, and Turgut Önder, eds.), 2017, pp. 86-111.

[Ven15] Sushmita Venugopalan, Vortices on surfaces with cylindrical ends, Journal of Geometry and Physics 98 (2015), 575-606.

[Wit93] Edward Witten, Phases of $N=2$ theories in two dimensions, Nuclear Physics B403 (1993), 159-222.

Beijing International Center for Mathematical Research, Peking University, BeiJing, China, And, Department of Mathematics, Princeton University, Fine Hall, Washington Road, Princeton, NJ 08544 USA

E-mail address: tian@math.princeton.edu

Department of Mathematics, Princeton University, Fine Hall, Washington Road, Princeton, NJ 08544 USA

E-mail address: guangbox@math.princeton.edu 